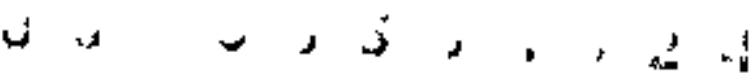

13 Lawrence Berkeley Laboratory UNIVERSITY OF CALIFORNIA

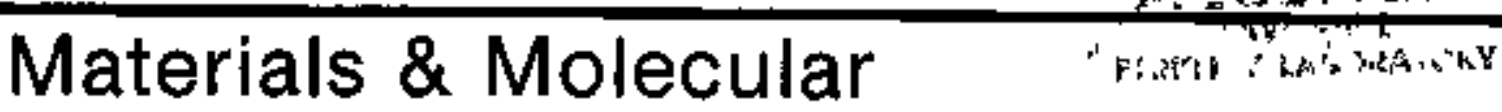 Research Division LeC - 14/9
}

THE LOOP-DRIVEN GRAPHICAL UNITARY GROUP APPROACH TO THE FLECTROH CORRELATION PROBLEM, INCLUOING CONFIGURAT ION INTERACTION ENERGY GRADIENTS

Bernard Robinson Brooks

(Ph. D. thesis)

-

\section{For Reference}

September 1979

\section{Not to be taken from this room}

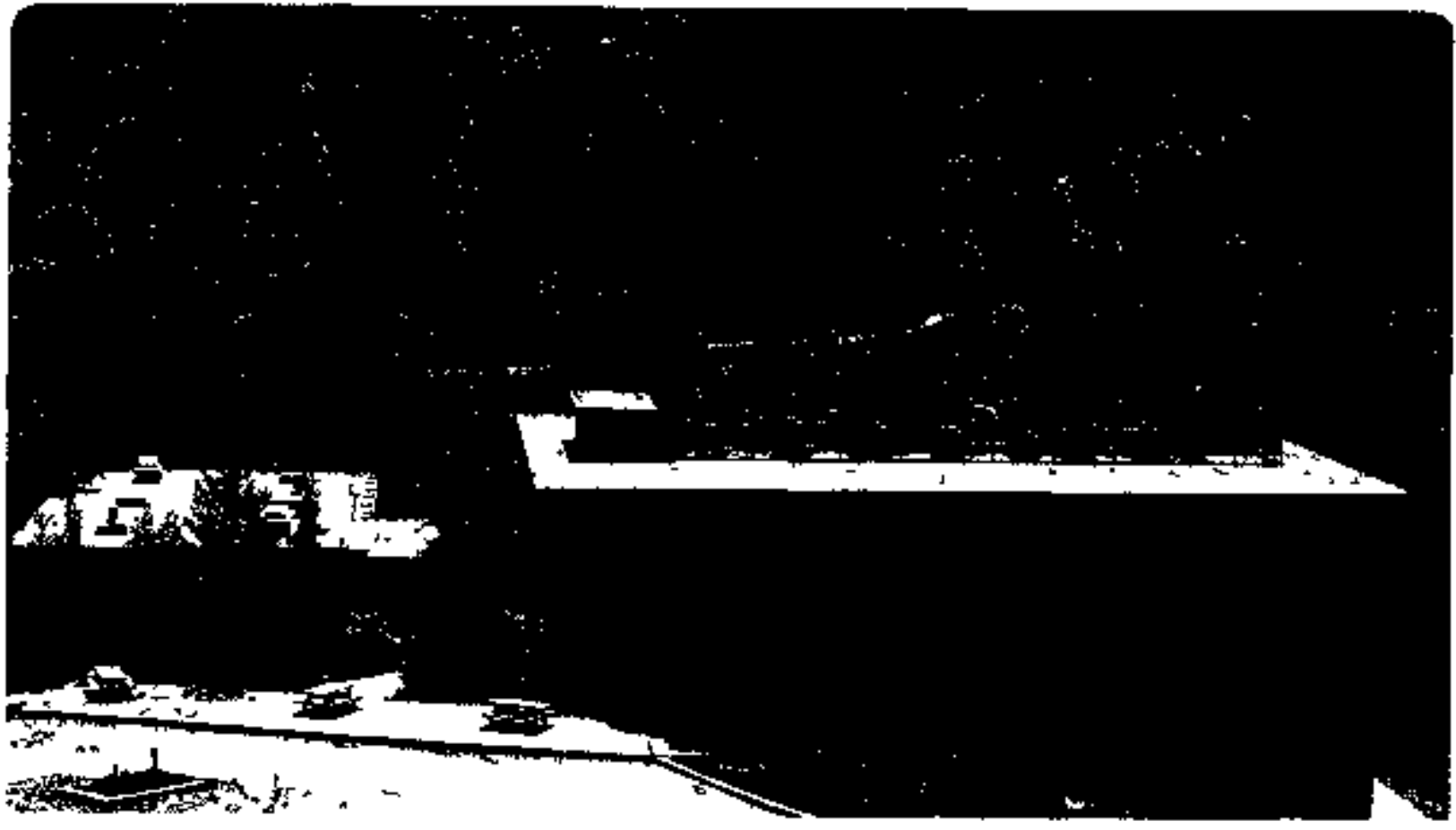

Prepared for the US Department of Energy under Contract W-7405-ENG-4g 


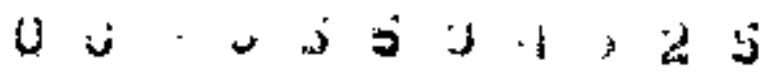

LBL -9998

\title{
The Loop-dxiven Graphical Unitary Group Approach \\ to the Electron Correlation Problen, Including Configuration \\ Interaction Energy Gradients
}

\author{
By \\ Bernard Robinson Brooks \\ Lawence Berkeley Laboratory, Untversity of Californ1a \\ Berkeley, CA 94720 \\ ABSTRACT
}

The Graphical tnitary Group Approach (GUGA) has beell cast irito an extraordinarily powerful form by restructuring the Haniltonian in terms of Ioop types. This allows the adoption of the loop-drivon formulation which illuminates vast numbers of previously unapprectatod rejationships between otherwise distinct hamiltonian matrix elentents. The theoretical/methodological contributions mate here include the development of the Joop-driven formula generation algorithas, a solution of the upper walk problem used to develop a loop breakdown algoritha, the restriction of configuration space employed to the multireference interacting space, and the restructuring of the Haniltonian in texms of looy types. Several other developments are presented and discussed. Anong these developmetsts are the use of new segment. coefficients, improvements in the loop-driven agorithr, implicit generation of loops sholly within the external space adapted within the framework of the loop-driven methodology, and comparisons of the diagonalization tape nethod to the disect methud. It is also shown how it is possible to implement the GUCA method without the timeconsuning full (m) four-irdex transformation. A particularly promising new direction presented here involves the use of the GUift mothodology to obtain one-electson and two-electron densty throtices. Once those 
are known, analytical gradients (first lerivatives) of the CI potential energy are easily obtained. Several test calculations are examined in detail to illustrate the unique features of the method. A1so included is a calculation on the asymetric $2^{1} \mathrm{~A}^{\prime}$ state of $\mathrm{SO}_{2}$ with 23,613 configurations to demonstrate nethods for the diagoralization of very large natrices on a minicomputer. 
To Terri

$"$ 


\section{ACKNONLEDGEMENTS}

Special thanks are due to Fritz Schaefer for sharing wth we a portion of his wealth of knowledge, for encouraging ne to work independently, and for allowing me to maintain ny noctumal habits. I an extrenely grateful to Professor Shi Shavitt for introdueing ne (and the world) to the Graphical Unitary Group formalism. I also thank Professor Shavitt for many invaluable discussions and communications throughout the development of this nethad. I an indebted to Professor Nick Handy for convincing me the CI gradients were feasible. I also wish to thank Professor Handy for presenting nany of the equations expressed in the gradient section as well as proposing algorithms to solve them.

I an also indebted to Bill Laidig and Patul Saxe whose excellent work and great effort enabled the completion of the second generation of Unitary Group programs and CI gradient prograns. I am also hopeful for the future, as the work here is not presented as a conclusion, but only a picture of a developing method that will certainly be carried much further by them and others. I also thank John Goddard, Per Siegbahn, Professor Paldus, Michel Dupuis, Richard Stratt, Ross Wetmore, Sigrid Peyerimhoff, and Yukio Yamaguchi for many invaluable discussions.

I thank Carol Hacker for much help throughout my four years at Berkeley (and even after leaving). I especially thank ny wife Terri who put more work into this thesis than I did.

This research was supported by the U.S. National Science Foundation, Grant CHE-7622621, and by the Division of Chemical Sclences, Offlce of Basic Energy Sciences, U.S. Department of Energy under contract No. W-7405-Eng-48. 
$0 ;: 2 ; 53.4,25$

TABLE OF CONTENTS

1. Introduction ................... 1

II. Review of Theory ................. 6

A. The Paldus Representation ........... 7

B. The Shavitt Representation .............9

C. Matrix Elements of the Unitary Group Generators . . . . 12

III. Nethods and Developnents of the Unitary Group Approach CI . . 16

A. The Interacting Space . . . . . . . . . 17

B. The Multireference Space . . . . . . . . . . 22

c. Spatial symmetry ................ 25

D. The Importance of Loop Types . . . . . . . . . . . 28

E. The Loop-driven Methodology . . . . . . . . . . 33

F. The Loop Searching Master Table ...........37

G. Integral Sorting and Storage . . . . . . . . . 43

H. The Loop Breaktown Algorithon (Solution of the Upper

Walk Probleni) . . . . . . . . . . . . . 47

I. Implicit Treatment of the Extermal Space ........ 50

J. Diagonalization Tape vs. Direct Method . . . . . . . . 52

K. Large Matrix Diagonalization Method ........... . 54

L. Density Matrix Analysis .............57

M. Avoidance of the Full Integral Transformation . . . . 58

IV. Aralytical Gradients for cr Energies . . . . . . . . .66

A. Theory of CI Energy Gradients .............65

B. Generation of the One- and Two-Particle Density Matrix . . 70

C. Transformation and Partial Transformation of the Two-

Particle Density Matrix . . . . . . . . . . 72

D. Generation and Use of the Lagrangian Matrix ...... 76 
E. Generation and Use of the Integral Derivatives . . . . 80

F. Coupled Perturbed Hartree-Fock . . . . . . . . . . 81

V. Graphical Unitary Group Approach CI and CI Energy

Gradients as a System of Programs . . . . . . . , 87

VI. Applications . . . . . . . . . . . . . . . 90

A. Exauples and Timing Results . . . . . . . . . . . 91

B. Vertical Electronic Spectrum of Ketene . . . . . . . . . . 94

VII. Concluding Remarks . . . . . . . . . . . . . . . . 96

References ......................... 97

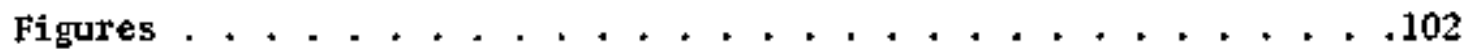

Examples ...........................110

Tables ........................... . . . . . . . . . .

The Loop Searching Master Table... . . . . . . . . . . 118 
U.

I. INTRODUCTION

The importance of electron correlation in quantum mechanical predictions of chemical interest can hardly be overestinated. It has been abundant $1 y$ documented that correlation effects can greatly alter theoretical predictions of bond energies, ${ }^{1}$ activation energies, ${ }^{2}$ and spectroscopic electronic energy differences, ${ }^{3}$ to cite three of the most iaportant examples. For this reason, much theoretical and computational effort has been justifiably expended in recent years in the development of new approaches to the correlation problem.

Fortunately, the past decade has witnessed great progress in the theorist's ability to describe electron correlation in a practical but rigorously variational manner. Most of the new methods are either variants of, ox in some way equivalent to, the standard nethod of configuration interaction (CI) ${ }^{4}$ Some of the most successful currently available techniques are the pair natural orbital (PNO) $\mathrm{CI}, 5,6$ direct CI methods, ${ }^{7-9}$ the method of self-consistent electron pairs (SCEP), 10 and a whole range of sophisticated general CI nethods. 11-19 In practical terns, these advances mean that a nearly complete solution of the correlation problem is now feasible for essentially any molectile for which ab initio self-consistent-field (SCF) wave functions could be obtained ten years ago. From another perspective, one can now approach the correlation problem for the benzene molecule $\left(\mathrm{C}_{6} \mathrm{H}_{6}\right)$ at a level of theory that was barely possible for methylene $\left(\mathrm{CH}_{2}\right)$ a decade ago. 20 Rather than satisfying the desires of chemists to use increasingly sophisticated quantum necharical techniques, the above developments have instead whetted appetites for further forays into even more 
complicated chemical problens. Thus the need for ever more powerful theoretical methods is likely to be with us for some time to come. One of the most promising ideas advanced in the past several years is the Graphical Unitary Group Approach (GUGA). Actually the unitary group mathenatical fornalism has been available for more than a decade, 21 and some of it for much longer. ${ }^{22}$ Hawever, it was by no means obvious that this fortalism had any particular advantage over existing variational many body techniques. The latter situation has been changed by the recent research of Paldus ${ }^{23}$ and of Shavitt ${ }^{24}$ on the adaptation of the unitary group approach to electronic structure problems. Indeed the present work was inspired by a series of lectures recent $2 y$ given by Shavite ${ }^{25}$ at Livenoore.

The formal basis of the tuitary group approach is the fact that a particular Gelfand-zetlin canonical basis spans the space of spinadapted many-electron functions for a given number of electrons and total spin s. ${ }^{2 I}$ This in itself is not terribly novel since there are many available techniques for the generation of spin eigenfunctions, e.g. the genealogical method, ${ }^{26}$ use of projection operators, ${ }^{27}$ and diagonalization ${ }^{28}$ of $\mathrm{s}^{2}$. The power of the graphical unitary group approach, as developed by Paldus ${ }^{23}$ and by Shavitt, 24,25 1ies with the tramendous amount of insight it provides into the structure of the Hamiltonian matrix $\mathrm{H}$. This structure is not only an edifice of mathematical beauty but also a practical tool for the calculus of matrix element evaluation.

Taking the work of Shavitt as our starting point, we present here the first implementation of the graphical ${ }^{24}$ unitary group approach 


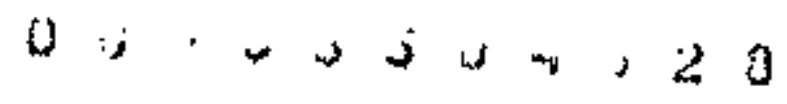

for molecular electronic structure problems. The nost critical contribution of our own work is the development of the loop-driven methodology. This method of generating the Hamiltonian contributions illuminates previous1y undiscovered relationships between otherwise distinct Hamiltonian natrix elements. Other theoretical contributions made here In addition to the development of the loop-driven algorithm are a solution of the upper walk problen, the restriction of configuration space employed to the multireference interacting space 29 suggested by second-order perturbation theory, and the restructuring of the Hamiltonian in terns of loop types. Within the Shavitt framewoxk a number of additional methodological modifications and impovenents have been rade to haxness the formalisn into a sequence of functioning algorithas. To demonstrate the power of the unitary group approach, it has been applied to a series of probtens, several of which were beyond the capabilities of our previous large scale configuration interaction (CI) techniques. 19,30 In fact for large, general, nultireference configuration interaction (CI) problens, our unitary group methods require only a fraction of the computation time necessary for state-of-the-art conventionel CI techniques. 19 when one realizes that these conventional methods have been perfected over a period of wore than 25 years, 4,31 the nagnitude of this echievement becomes apparent. Of colrse the basic forsalisn of the unitary group approach (UCA) has been avallable for more than a decade. 21

The development of analytic gradient techniques for the investigation of potential energy surfaces hes been one of the most inportant recent developments in electronic structure theory. ${ }^{32}$ Wheh of the 
early advancenent in this area is largely due to the work of Putay, ${ }^{33}$ who presented an expression for the forces on ali of the auclet for closed-shell self-consistent-field theory. His techniques have been used mainly for prediction of force constants and equilibrium geometries. Other more recent ab initio gradient techniques and methods are due to the further work of Poppinger, ${ }^{34}$ Schlesel, 35 Kormornicki, ${ }^{36}$ Duputs and King, ${ }^{37}$ Pople, ${ }^{38}$ Hehre, ${ }^{39}$ and by Goddard, Handy and Schaefer. ${ }^{40}$ with these methods, energy derivatives with respect to nuclear position can be obtained for a wide range of electronic wavefunctions, including open-shell restricted Hartree-Fock, umrestricted Hartree-Fock, two configurgtion MCSCF wavefunctions, and for second order Noller-Plesset perturbation theory. Notably missing fron this list are analytic gradient methods for large scale configuration interaction wavefunctions.

We present here the new nethods required to obtaln analytic gradients for general large scale CI calculations, we weIl as a description of the first implenentation of these methods. The most inportant contribution of this work is the use of the loop-driven algorithn to generate the two-particle density natrix. Another important contribution is in the area of deternining the gradient contribution for perturbations in the CI expansion basis. pople ${ }^{41}$ has recently developed an efficient iterative computational method for solving the coupled-perturbed Hartree-Fock (CPHF) equations given by Gerratt and Mills 42 for restricted Hartree-Fock wavefunctions. We have improved this technique to simultaneously solve the CPHF equations for all $3 \mathrm{~N}$ nuclear perturbations (where $\mathrm{N}$ is the number of nuclei) 


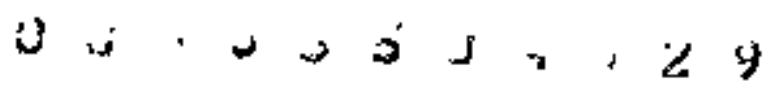

with a further reduction of necessary work. 


\section{REVIEW OF THEORY}

There are two rather distinct aspects of the GUGA nethod, the first being the generation of the distinct row table (DRT), which completely determines the configuration space that is included in the CI. The DRT can be as complex as necessaty to describe any predetemined configuration set. The second aspect is the utilization of the DRT as a backbone or template to generate all of the loops which define the Hanlltonian natrix is a conpact symbolic form. Each loop defines a set of equivalent matrix element contributions. The loop-driven a1goritho is used to generate the loops in a rapid nanner. 
$06: 2 ; 5 ; 4,30$

A. The Paldus Representetion

Each Gelfand state ${ }^{22}$ (or configuration) can be represented by a three colum array of non-negative integers referred to as a Paldus array or tableau that spans the nuber of nolecular orbitals (n) used in the CI,

$$
[p]=\left[\begin{array}{lll}
a_{n} & b_{n} & c_{n} \\
a_{n-1} & b_{n-1} & c_{n-1} \\
\vdots & & \\
a_{1} & b_{1} & c_{1} \\
0 & 0 & 0
\end{array}\right]
$$

For this representation the top row determines the electronic state, and each other row, where

$$
a_{i}+b_{i}+c_{i}=i
$$

represents a particular irreductble representation of the umitary group U(i). There are only four ways (or cases) in which one row (i-1) of any paldus array can differ from the next row (i), which deternines the accupancy of orbital i for that particular corresponding Gelfand state. The four cases are identified by case numbers $s_{1}$ which can be sumarized by:

\begin{tabular}{cccccc}
$\mathbf{s}_{\mathbf{i}}$ & $\Delta \mathrm{a}_{\mathbf{i}}$ & $\Delta \mathrm{b}_{\mathbf{i}}$ & $\Delta \mathrm{c}_{\mathbf{i}}$ & $\mathbf{n}_{\mathbf{1}}$ & \multicolumn{1}{c}{$\mathbf{s}_{\mathbf{i}}$} \\
\hline 0 & 0 & 0 & 1 & 0 & 0 \\
1 & 0 & 1 & 0 & 1 & $1 / 2$ \\
2 & 1 & -1 & 1 & 1 & $-1 / 2$ \\
3 & 1 & 0 & 0 & 2 & 0
\end{tabular}.


Here $\Delta a_{i}=a_{i}-a_{i-1}$ and $n_{i}$ is the number of electrons in orbital $i$. Case o results when orbital $i$ is unoccupied. Cases 1 and 2 result from the different spin couplings (referred to as alpha and beta coupled respectively) of the singly occupied orbital 1 , and case 3 results fron a doubly occupied orbital $i$. It is clear fron this that

$$
a_{i}=\frac{l}{2} N_{i}-s_{i}
$$

and

$$
b_{i}=2 s_{i},
$$

where $N_{i}$ is the number of electrons in the first $i$ orbitals and $s_{i}$ is the spin of this subgroup. For a given top row, all possible Paldus arrays that can be generated correspond to the configurations of a fu11 CI treatment. Perhaps a simpler way to represent each Gelfand state is by a series of $n$ case values $\left(s_{1}, s_{2}, \ldots, s_{n}\right)$ where $n$ is the number of orbitals. There is a one-to-one correspondence between these case arrays and complete, orthonornal, spin-adapted Gelfand states. 


\section{B. The Shavitt Representation}

In the graphical representation developed by Shavitt 24,25 primarily for illustrative purposes, each different case value . corresponds to a different slope line segment (or arc) that spans one orbital. A vertical arc represents an moccupled orbital $\left(s_{i}=0\right)$. A sharply slanted arc represents a dotbly occupied orbital $\left(s_{1}=3\right)$ and the two singlet couplings are identified by differing internediate slopes. Then a series of these arcs are chained together as described by the case array $s_{i}$, one forms a path (or walk) that untquely defines that Gelfand state. One of the most renarkable features of these paths is that when any two such paths of a given spin state are superimposed so that their tails (bottom) coincide, their heads (top) must also coincide. Their paths may also coincide at intertuediate points. This suggests superimposing all of the Gelfand states desired for a given CI calculation. When this is done one obtains a Shavitt graph (or master graph as referred to previously). The numerical analog of a Shavitt graph is referred to as a distinct row table (DRT). A sample Shavitt graph is depicted in Figure 1. The corresponding DRT for this case is shown in Table $I$. The Shavitt graph ${ }^{24,25}$ is identified by a series of vertices (distinct rows) and ares. The rows of any level $i$ correspond to the possible rows of the PaIdus arrays of that Gelfand basis at that same level $i$. The Index $j$ refers to a given distinct row within 1 , such that

$$
i=a_{i j}+b_{i j}+c_{i j}
$$

Each level $i$ is connected to the next lower level i-1 by the occupancy 
of orbital i. Each row of level $i$ can relate to up to four rows of Ievel $i-1$ by the four different cases $\left(s_{1}, s_{1}=0,1,2,3\right)$. Any such valid relation corresponds to an arc. Each arc can be represented by the three indices $(i, j, s)$ which will miquely determine its position within the DRT. Each vertex (at level i) identifies a particular component of Gelfand states containing this vertex and is deternined by the number of electrons and total spin of these electrons in the first i orbitals. The Shavitt graph is al so two-rooted in that single points determine its head or tail. The graph tail corresponds to the origin and the graph head uniquely deternines the number of electrons and the spin state. Each possible walk represented by a series of $n$ arcs fron the graph head to the graph tail corresponds to a particular configuration. In general the number of different arcs and distinct rows is much smaller than the nuber of configurations. There is a one-to-one correspondence between these walks and the Gelfand states of the Getfand-Zetlin basis. The selective elimination of certain arcs is used to generate a subset of the configurations of the full CI, such as all singly and doubly excited configurations from a given reference.

There is also a lexical ordering of states such that $m^{*}<m$ if and only if $s_{i}<s_{i}$ at the highest level, where $s_{i} \neq s_{i}$ for those states. Here $i$ refers to the state index and $s_{i}$ refers to the case values $\left(s_{i}=0,1,2,3\right)$. For each distinct row there are a number of $x_{r}$ of lower walks (possible paths to reach the graph tal1) and also a number of $\bar{x}_{r}$ of upper walks. The lexical ordering of states provides that for any upper walk fron a given distinct row all states corresponding 


$$
0+3, j 5+32
$$

to the different lower walks are sequential. Each given now of the Shavitt graph or DRT is also given an arc weight $(y)$ for each valid arc. The index $n$ for any state can be expressed as a sum of the arc weights over all the levels. Table I gives a sample DRT with all of the mentioned features.

The ntuerical analog of the Shavitt graph is the DRT (distinct row table) which in its simplest form is made up of different distinct rows in the Paldus representation. 
C. Matrix El ewents of the Unitary Group Generators

Matrix eloments between two Gelfand states can be expressed as

$$
\begin{aligned}
& \left\langle m^{\prime}|H| m\right\rangle=\sum_{i, j}\langle\dot{I}|\hat{i}| j\rangle\left\langle m^{\prime}\left|E_{i j}\right| m\right\rangle
\end{aligned}
$$

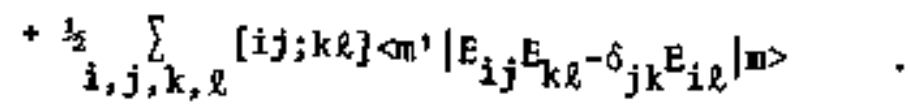

The spin tndependent generator $E_{i j}$ is given by

$$
e_{i j}=\sum_{\sigma} x_{i \sigma}^{\dagger} x_{j \sigma}
$$

where $x_{i \sigma}^{\dagger}$ and $x_{i \sigma}$ are creation and annihilation operators for an electron in orbital $i$ and spin state $\sigma$. The generator $E_{i j}$ when operating on a Gelfand state, renoves an electron from orbital $j$ and adds one to orbital $i$. Other propexties of these generators are:

$$
\left\langle\pi^{\prime}\left|E_{i i}\right| n\right\rangle=\delta_{n^{\prime}} n_{i},
$$

where $E_{1 i}$ is termed a weight generator, and

$$
\left\langle n^{\prime}\left|E_{i j}\right| n\right\rangle=\left\langle m\left|E_{j i}\right| n^{\prime}\right\rangle=0, \quad\left(i<j, m^{\prime} \gg m\right)
$$

due to the lexical ordering of states.

Direct formulas for the evaluation of matrix elenents of any generator have been derived by Gouyet et a1, ${ }^{43}$ and by Drake and Schlesinger, 44 and these lead to a factorization of generators and generator products into segnent contributions. Thus each different segment shape has certain coefficients associated with it that are determined by generator factorization. This factorization is one of 
the essential features that distinguish the GuGA method fron other unitary group CI nethods.

The natrix elenents of the generators are deternined by a factorization (of the natrix element) over the levels between and including the indices of the generator or generator product. The oneelectron generator matrix elenents can be given by

$$
\left.\measuredangle m \cdot\left|E_{p q}\right| m\right\rangle=\prod_{i=p}^{q} H\left\langle T_{i}, s_{i}^{i} s_{i}, A b_{i}, b_{i}\right\}
$$

where the different W segment values are simply predetermined coefficients for each possible segnent shape. Whenever two configurations in the graphical representation $\mathrm{n}^{\prime}$ and $m$ have a nonzero watrix element such that $\left\langle m\left|E_{j i}\right| m>\neq 0\right.$ (where $f>i$ ) are superimposed, they must coincide at all levels above level $j$, (referred to as an upper walk) and also coincide at all levels below level i (referred to as a lower walk). The distinct row at level $j$ is referred to as the loop head. The distinct row at level i-l is referred to as the loop tail. The separate walks between the loop head and loop tał are referred to as the $\mathbb{k}$ and $n$ ' branches of the loop. Within the loop each level comects with the next lower level by a given loop segment. For single generators there are only 18 valid segment shapes (or types). For generator products there are many nore such segnent shapes. The body of this loop is depicted by the behavior of the segment shapes between levels $i$ and $j$ inclusive. The coefflcient of integral $\langle i|\hat{h}| j\rangle$ is detexmined only by the body of the loop itself, thus different configuration pairs that share the sane loop body with different upper and lower walks will have the same one-electron 
integral contribution. By defining a loop only by its body, the number of times this given loop will contribute to the Haniltonian is given by the product of the number of upper walks from the loop head and the number of lower walks fron the loop tail $\left(\bar{x}_{h} \cdot x_{t}\right)$. A sample one-electron loop is depicted in Figure 2.

The two electron integral contribution of the Hamiltonian is given by

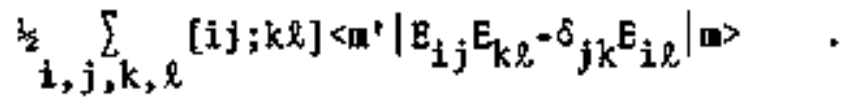

In our preliminary implementation the matrix element of generator products was determined by a factorization requiring internediate stat es such that

$$
\left\langle m^{\prime}\left|E_{p q} E_{r s}\right| m\right\rangle=\sum_{g^{\prime \prime}}\left\langle m^{\prime}\left|E_{p q}\right| n^{\prime \prime}\right\rangle\left\langle m^{\prime \prime}\left|E_{r s}\right| m^{\prime}\right\rangle .
$$

For levels where pq and rs do not overlap, the segment coefficients are identical to those of the single generator. An efficient recurstve schene was developed by Shavitt ${ }^{24}$ for the evaluation of these generator products within the overlap region. There are, however, several drawbacks to this approach (when colpared with newer approach), the most serious being that at any level within the overlap region up to three segment coefficients are needed when the loop-driven algorithm is used. Another difficulty is that the $\delta_{q r_{p s}}$ term is not included which results in calculation of extra contributions that cancet 2ater. 
Work by Paldus ${ }^{45}$ and Shavitt ${ }^{46}$ since the completion of our prelininary implementation have suggested that the matrix element generator products be represented by

$$
\begin{aligned}
& \left\langle m^{\prime}\left|\varepsilon_{p q, r s}\right| m\right\rangle=\left\{\underset{i\left(n_{0} \text { overlap }\right)}{\Pi\left(T_{i}, s_{i}^{\prime} s_{i}, \Delta b_{1}, b_{i}\right)} \cdot\right. \\
& \sum_{x=0,1} I_{i \text { (overlap) }} W\left(T_{i}, s_{i}^{\prime} s_{i}, \Delta b_{i}, b_{i}, x\right)
\end{aligned}
$$

where $\varepsilon_{\mathrm{pq}, \mathrm{rs}}$ is given by

$$
E_{p q, r s}=E_{p q} E_{r s}-\delta_{q r} E_{p s}
$$

This representation is superior to the previous nethod for several reasons. One obvious reason is that each segment in the overlap region now has at most two coefficients. The $x=0$ branch and the $x=1$ branch elucidate the relationship between direct and exchange terms for integrals that are related by index permutation. When the use of loop types is employed where two integrals are involved, the coefficients of both integrals can be determined by the sane two segment coefficients in the overlap region, resulting in a further reduction of work. One final reason that coefficients defined in this nanner are superior is that the $\delta_{\mathrm{q} \mathrm{r}^{\mathrm{E}} \mathrm{p}^{\mathrm{B}}}$ term is included, elininating ali of the cancellation of repeated terms present in the earlier version of the method. The implementation of these new coefficient values is one of the major reasons for the factor of two reduction of computational times from those of the already impressive preliminary implementation. 
III. METHODS ANO DEVELOPNENTS OF THE UNITARY GROUP APPROACH CI 


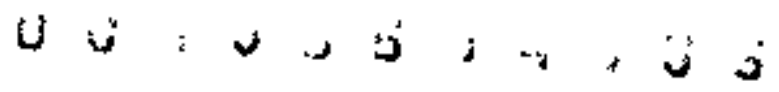

A. The Interacting Space

For any general $\mathrm{CI}$ nethod to be useful it nust be able to include those classes of configurations that are itoportant without being required to also include a vast set of insignificant configurations. One such inportant distinction concerns the Hartree-Fock interacting space, ${ }^{29}$ those configurations that potentially interact with the references, conpared with the full spin configuration space. Experience has demonstrated ${ }^{47}$ that these additional configurations within the full spin space are usimportant in terns of correlation energy and electronic properties, and their exclusion substantially reduces the computational effort required for the CI calculation.

In 1 ts earliest electronic formulations, the effectiveness of the GUGA was only apparent for full CI wavefunctions. This capability was thereafter considerably extended by Shavitt 24,25 to cases involving different levels of excitation relative to a single reference configuration, our contribution in turn was the extension of the GUGA to the muttireference 1nteracting space, Since the teron "interacting space" is not widely understood in this context, a specific exanple is in order.

The restricted Hartree-Fock wavefunction for the triplet ground state of methylene $\left(\mathrm{CH}_{2}\right)$ is of the form

$$
1 a_{1}^{2} 2 a_{1}^{2} 1 b_{2}^{2} a_{1} \alpha 1 b_{1} \alpha
$$

Now conslder a double excitation of the form $2 a_{1} 1 b_{2}+i j$. This gives rise to the now orbital occupancy 


$$
1 a_{1}^{2} 2 a_{1} 1 b_{2} \text { i j } 3 a_{1} 1 b_{1}
$$

with six unpaired spins. These six mpaired spins give rise to no fewer than nine triplet (S=1) spin eigenfunctions. Most conventional $C I$ methods require either none or all of these configurations to be included variationally. ${ }^{4}$ However, this 9 -dimensional spin space can be partitioned 29 in such a way that only two of the configurations have nonvanishing Hamiltonian matrix elenents with the Hartree-Fock reference configuration (16). The two "interact1ng" configurations are of the form

$$
1 a_{1}^{2}\left[2 a_{1} 1 b_{2} \text { i } j \Rightarrow{ }_{A_{1}}\right] 3 a_{1} \alpha 1 b_{1} \alpha
$$

In which the four arbitals in brackets have been coupled in the two possible ways yielding a singlet $(\$=0)$ state.

The seven configurations neglected above are exactly those which do not contribute in second-order perturbation theory. Further, numerical tests on methylene and other aolecules have shown that the interacting space does include all energetically inportant configurations. ${ }^{47}$ That is, although the interacting space includes typically less than half of all symetry-adapted configurations in a given double excitation space, it yields $\approx 99.9 \%$ of the correlation energy due to the conplete space.

Consider a singles and doubles CI calculation from a single doublet reference configuration that can be described by a single Sister deteminant

$$
1 \mathrm{a}^{2} 2 \mathrm{a}^{2} 3 \mathrm{a}^{2} \ldots \mathrm{nga}
$$


Host of the doubly excited conflgurations of this calculation (assuming a large enough problem) will have five unpaired spins. For a given orbital occlpancy the full $\mathrm{s}-1 / 2$ spin space will consist of five configurations. These conf1gurations can be expressed as linear conbinations of the ten possible $M_{s}=1 / 2$ slater detertinants. On the other hand, the interacting space will consist of only two of these configurations and can be represented by a linear conbination of six of the ten possible slater deteminants. (Four of the unpaired orbitals are singlet coupled and the fifth mpaired spin, corresponding to the unpaired reference orbital, is restricted to be of the same spin occupation as in the reference.)

lising the clebsch-Gordan expansion, the coefficients of the five Gelfand states expressed over determinants (ignoring the doubly occupied and unoccupied orbitals) are given in Table II. By inspection the first and second conflgurations are the only patr of configurations that can select the interacting space from the full spin space. Notice that for this combination the fifth unpaired spin must always be alpha occupied. This must correspond to the unpaired orbital of the reference configuration. In order to have the fifth unpaired spin to be the last for all possible double excitations that orbital must be the last (or the top) orbital of the distinct row table.

For higher spin states, such as triplets, to generate the interacting space all of the spin restricted orbitals must be at the top of the distinct row table. For a single open-shell singlet reference conflguration the spin restricted orbitals have two possible positions on the DRT, at either extrene. For the sake of continuity 
we chose to put them at the top also. The renainder of the orbitals may lie anywhere within the rest of the DRT except that all of the doubly occupied orbitals should be grouped together and all of the virtual orbitals should be grouped together. This is done so that it Is easy to keep track of the excitation levels, without adding additionat dinenstons to the DRT. For an optimized method the larger of these two blocks should be at the botton of the DRT. For calculat1ons of double zeta quality or better, the virtual space is larger than the doubly occupied space; thus within the DRT the orbitals are ordered (from top to botton): the active space, the doubly occupied space, and the virtual space. Renoved from the DRT are the frozen core orbitals and the deleted virtual orbitals.

To implement the use of the interacting space, each row of the DRT is given a type (T) classification. This value, which corresponds to the number of excitations accounted for, determines the maximum number of electrons that can be excited into the virtual space for all walks containing this distinct row. For a singles and doubles CI, a value of $T=0$ inplies that two electrons may be excited into the virtual space, because no excitations have occurred in the active space. A value of $T=1$ or $T=2$ implies that one or no electrons respectively may be excited into the virtual space. The $T$ value is only meaningful for those rows of the DRT that are above the interface between the doubly occupied and virtual space. For the one reference doublet interacting space, if the orbital that is unpaired in the reference is doubly occupied (s=3) or beta coupled (s=2), the T value is set to one, because either of these occupations accounts for one of the two 


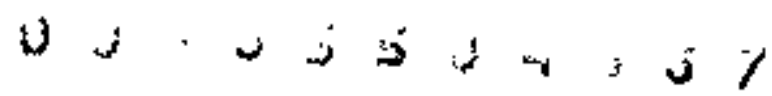

possible excitations. The $T$ values are determined by the active orbitals at the top levels of the DRT, and the same $T$ values renain unchanged along any walk within the doubly occupied space. All potential excitations are compared with the $T$ value to determine if that exc1tation is valid.

One problem with this approach to the interacting space is that it is possible for the two branches of a valid loop to end on two different rows that only differ by $\mathrm{T}$ values. The solution to this problem is to extend the loops for these cases until the rows colncide. This will occur at the level of the interface between doubly occtpied and virtual orbitals. This 1oop extension will not aiter the loop coefficient or the integrals that contribute to that loop, but is required so that the contribution will be added to the correct matrix elements. This extension is not a major conputational consideration since only a very snall percentage of the total loops will require this extension and all of the loops that require this extension are smal 1.

This simple algoritho can be used to generate the interacting space from higher spin states as well as from open-sheil singlet references. 


\section{B. The Multixeference Space}

Whereas aIl single and double excitations from a single reference configuration nay be adequate for some molecular systems, many of the more interesting chenical problens require dotble excitations from more than one reference for an adequate description. $3,11,12,15,20$ None of the other direct CI methods can handle these types of problens except for the most restrictive cases. ${ }^{48}$ on the other hand the graphical unitary group approach is ideally suited for such problems because the configuration space is determined only by the DRT, which can be as complex as desired, to handle almost any problem.

For nost cases the active orbltals, those orbitals that are either singly occupied or occupied differently by different references, must be adjacent within the distinct row table. For cases where the full spin space is desired, the active orbitals may be anywhere within the DRT, but with the nost opt1mized method they should be between the doubly occupied and virtual space. Whenever the interacting space is also required the active orbitals must be placed at the top of the DRT. If the active orbitals are at the top of the DRT, for either full spin space or multireference interacting space calculations. then the same $T$ values described in the interacting space section can be used to generate the multireference configuration space, For more complex multireference calculations, several different $T$ type parameters must be used. Typical1y the nuber of $T$ type parameters must be the same or less than the number of reference configurations, but these additional paraneters are only needed within the active orbital space of the DRT. If the active orbitals are placed between the occupled 
and virtual space, then another algorithm must be used. The system in its present form always restricts the active orbitals to be at the top of the DRT.

The method for implementing a given multireference calculation consists of two steps. First, a small model DRT without symbetry must be created describing the desired multireference configuration space; and secondly, an algorithm must be developed that can reproduce this model as well as the desired multireference configuration set in the general case. Once a given case has been worked out, the developed algorithe may be added to the rest of the system. The complexity of the model increases rapidly as the number of references increases, which can make this nethod quite difficult even for some three reference calculations. Table III gives an example of a model DRT for the interacting space fron two open-shell singlet reference configurations. An important future advancement of the GUGA method will be the automation of the multireference configuration space generation. Gnce this has been done the capabilities of this method in terms of types of calculations will be almost linitless.

The algorithn that will make the GUGA method completely generaI 1s still in the design stage. In its current form it can roughly be broken up into the following steps:

1. Generate the distinct row table (DRT) with some or nany unwanted configurations included.

2. Generate the indexing array.

3. Strike out unwanted configurations from the indexing array.

4. Restructure the indexing array in such a way as to maximize 
similarities (this will result in the simplest final DRT).

5. From this new indexing array generate the compressed form of the indexing array.

6. Using these two arrays and the old DRT, generate a new DRT that has only the wanted configurations present.

7. Use this DRT for the rest of the CI.

This algorithri currently works for unrealistically simple cases, but its generalization in an efficient manner that does not require a great deal of intermal storagej will be rather difficult. 


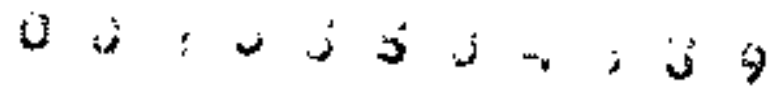

\section{c. Spatial Symmetry}

Orbital or spatia1 symetry can be treated in several ways within the structure of the GUGA method. At the simplest level symmetry can obviously be ignored. This will result in an unnecessarlly large formula tape as well as a much larger secular equation than necessary when symmetry is present. For example, if we are studying the ${ }^{3} B_{2}$ ground state of methylene $\left(C_{2 v}\right.$ point group), the number of unique configurations nay be increased by a factor of 5 if spatial syometry ts ignored.

At the first level of sophistication, symetry may be used only to eliminate those loops where the contributing integrals will be zero by symetry. This approach will result in the smallest possible formula tape and fastest formula tape generation (if inplemented properly); however the secular equation will stili be large. The Hamiltonian matrix will be blocked with regards to the different irreducible representations, but the entire natrix will present itself to whatever diagonalization scheme is used. Since most diagonalization schemes require at least two real vectors in core at any one time, this approach is only feasible for large core machines. Even if some clever diagonalization scheme is devised that can reduce the problem of the Iarge secular equation, it seems unlikely that the snall savings in the formula tape (compared with other symetry treatments) will warrant the increased difficulty in the diagonalization, especially if the formula tape is to be used more than once.

An innovative method proposed by Shavitt ${ }^{49}$ left the DRT unchanged fron that without spatial symonetry, except that for each row there are 
stored separate configuration counting indices for each symetry type of a nondegenerate point group. This results in the elinination of al1 configurations that are of a different irreducible representation than that of the reference. Each loop on the formula tape will have separate indexing indices for each symetry structure of that loop. We have carried this idea further by treating the different symmetry species of any row as if they were unrelated and only including those that have both a nonzero number of upper and lower walks. This provides for a simpler algorithm when constructing the loops except that a given loop may have to be created as many times as there are irreducible representations in the symetry point group. Instead af making each row more complex, additional rows are added to the DRT by giving each row a symmetry type classification, where the synmetry type of any row is the product of the inreducible representations of al1 singly occupied orbitals ( $s=1$ or $s=2$ ) of any lower walk. In principle this approach could multiply the complexity of the DRT by the number of symetry types. This is an important consideration since both the computational effort required as well as the formula tape Iength is heavily dependent on the complexity of the DRT, The number of different symmetry type classifications at any level of the DRT can be expressed by

$$
n_{\Gamma}(i)=\min \left[P_{u}(i), P_{\hat{x}}(i)\right]
$$

where $P_{u}$ and $P_{\ell}$ are the number of different symetry products of all possible combinations of the irreducible representations of all of the upper or lower orbitals, respectively. This number can vary greatly 
depending on the order of the orbltals with regards to symetry type. This suggests that the complexity of the DRT can be greatly reduced by a reordering of orbitals within each type of orbital space (active, doubly occupted, and virtual). The method adopted for our implenentation results in a rigorous minimization of

$$
\sum_{1=1}^{n} n_{\Gamma}(i),
$$

where $n$ is the number of levels. This is done by placing all of the symwetric oxbitals at the extremes of the DRT so that $\mathrm{n}_{\Gamma}$ of as many levels as possible will be unity. Similar considerations are used in the ordering of the rest of the orbitals. This is apparently also equivalent to the minimization of the number of distinct rows in the DRT. For favorable cases the DRT is only slightly mare complex than the DRT produced without symetry. 
D. The Importance of loop Types

The two-electron integral contribution of the Hamiltonian is

$$
\frac{1}{2} \underset{i, j, k, \ell}{[}[i j ; k \ell]\left\langle n '\left|\varepsilon_{i j, k \ell}\right| m\right\rangle \quad .
$$

The sum is over all ordering of the indices, and hence a given integral [ij;kl] where $i, j, k$, and $\ell$ are a11 different w111 appear eight times with coefficients in terms of generator products of:

$$
\begin{aligned}
& { }_{\text {r }} \varepsilon_{i j, k \ell} \quad{ }^{t} E_{k \ell, i j} \\
& { }^{1} E_{j i, k \ell} \quad{ }_{-3 \varepsilon} \varepsilon_{k \ell, j i}
\end{aligned}
$$

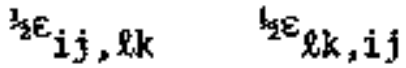

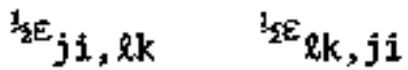

As demonstrated by Paldus ${ }^{50}$ and by Shavitt, ${ }^{24}$ since only matrix elements where $m^{\prime} \leq \mathrm{m}$ are of interest, the last four of these contributions are zero. Also since

$$
\varepsilon_{i j, k \ell}=\varepsilon_{k \ell, i j}
$$

the first pair of these contributions is identical and the second pair is also identical. The contribution of this integral may be expressed as

$$
[i j ; k l]\left\langle m\left|\varepsilon_{i j, k l}+\varepsilon_{j i, k \ell}\right| m>\quad(i<j<\ell, j \neq k<\ell)\right.
$$

A similar treatment of the remaining types of two-electron integrals 50,24 leads to the following results: 
U.

$$
\begin{aligned}
& {[i j ; j \ell]\left\langle m^{\prime}\left|\varepsilon_{j \ell, i j}+\varepsilon_{j i, j \ell}\right| m>\quad\{i<j<\ell\}\right.} \\
& {[i j ; i \ell]\left\langle m '\left|\varepsilon_{i j, i \ell}+\varepsilon_{i \ell, j i}\right| m>\quad(i<j<\ell)\right.} \\
& \left.[i \ell ; k \ell]<\mathbf{n}^{\prime}\left|\varepsilon_{i \ell, k \ell}+\varepsilon_{\ell i, k \ell}+\varepsilon_{\ell k, i \ell}\right| \nabla\right\rangle \quad\{i<k<\ell\} \\
& {[i j ; l \ell]<m^{\prime}\left|\varepsilon_{i j, l \ell}\right| m>\quad(i<j, i \neq l, j \neq l)} \\
& {[i \ell ; \ell \ell]\left\langle m^{1}\left|\varepsilon_{\ell \ell, i \ell}\right| m>\quad\{i<\ell)\right.} \\
& {[i i ; i \ell]\left\langle m^{\prime}\left|\varepsilon_{i j, i \ell}\right| m>\quad\right. \text { (i<l) }} \\
& {[i \ell ; i \ell]<\pi^{\prime}\left|\gamma_{2} \varepsilon_{i \ell, i \ell}+\varepsilon_{\ell i, i \ell}\right| m^{>} \quad \text { (i<l) }} \\
& {[i i ; \ell \ell]<m \cdot\left|\epsilon_{i i, \ell \ell}\right| m>}
\end{aligned}
$$

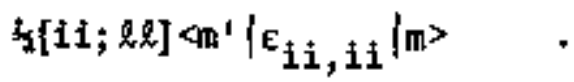

It should be mentioned here that this representation of integral contributions in terms of generators is not unique. Since the choice of representation will affect the loop types that are used in the loop generator, other representations should be considered.

A rearrangement of these equations leads to the important development of using loop types. By combining integral generator products that can contribute to the same matrix elenents (including the one-electron integrals), we find that there are fourteen different loop types.

$$
\begin{aligned}
& \text { 1) }\left\langle 0^{1}\left|[i k ; j \ell] \varepsilon_{k i, j \ell}+[i j ; k l] \varepsilon_{j i, k \ell}\right| m\right\rangle \quad(i<j<k<\ell) \\
& \text { 2) } \quad m||[i \ell ; j k] \varepsilon_{k j, i l}+[i j ; k \ell] \varepsilon_{i j, k \ell} \mid m>\quad(i<j<k<l) \\
& \text { 3) } m^{\prime}\left|[i k ; j l] e_{i k, j \ell}+[i \ell ; j k] \varepsilon_{j k, i \ell}\right| m>\quad(i<j<k<\ell) \\
& \text { 4) }\left\langle\mathrm{T}^{\prime}\left|[i j ; j \ell] \varepsilon_{j i, j \ell}\right| \mathrm{m}\right\rangle \quad(i<j<\ell) \\
& \text { 5) }\left\langle\pi^{\prime}\left|[i j ; j \ell] \varepsilon_{j \ell, i j}+[j j ; i \ell] \varepsilon_{j j, i \ell}\right| \omega\right\rangle \quad\{i<j<\ell\} \\
& \text { 6) <n' } \left.\left|[i k ; i l] \epsilon_{i k, i \ell}\right| m\right\rangle \quad(i<k<\ell) \\
& \text { 7) }<n^{\prime}\left|[i k ; i \ell] E_{i \ell, k i}+[i i ; k \ell] \varepsilon_{i i, k \ell}\right| m>\quad(i<k<\ell)
\end{aligned}
$$




$$
\begin{aligned}
& \text { 8) } \left.<m^{\prime} \mid[i \ell ; j \ell] \varepsilon_{i \ell, j \ell}\right\}^{m>} \quad(i<j<\ell) \\
& \text { 9) }<m '\left|[i \ell ; j \ell] \epsilon_{\ell i, j \ell}\right| m>\quad(i<j<\ell) \\
& \text { 10) }\left\langle\mathbb{I L}^{\prime}\left|[i \ell ; j \ell] \varepsilon_{\ell j, i \ell}+[i j ; \ell \ell] \varepsilon_{\ell \ell, i j}\right| m\right\rangle \quad(i<j<\ell] \\
& \text { 11) }\left\langle\mathbb{R}^{\prime}\left|[i \ell ; \ell \ell] \varepsilon_{\ell \ell, i \ell}+[i i ; i \ell] \varepsilon_{i \ell, i i}+\langle 1|\hat{h}| \ell\rangle E_{i \ell}\right| m\right\rangle \quad(i<\ell\} \\
& \text { 12) }\left\langle w^{\prime}\left|[i \ell ; i \ell] \varepsilon_{i \ell, i \ell} / 2\right| m>\right. \\
& \text { 13) }\left\langle m^{\prime}\left|[i \ell ; i \ell] \varepsilon_{\ell i, i \ell}+[i i ; \ell \ell] \varepsilon_{i i, \ell \ell}\right| m\right\rangle \\
& \text { 14) }\langle\mathrm{WI}|[\ell \ell ; \ell \ell] E_{\ell \ell, \ell \ell} / 2+\left\langle\ell|\hat{\mathrm{b}}| \ell>E_{\ell \ell} \mid \mathrm{m}\right\rangle \text {. }
\end{aligned}
$$

One obvious advantage of defining loop types in this manner is that loops now can represent a linear combination of integrals and this linear comblnation can be perforned at an early stage of the calculation. A schematic of each loop type is shown in figure $3 .^{46}$

Loop types 1, 2, and 3 are the most abundant, so most generated loop values will consist of a linear combination of two integrals. The importance of using loop types defined in this manner should not be underestimated. If loops were generated for each integral separately, not only would the effort required to generate the loops be doubled, but the same doubling would apply to the effort required each iteration of the diagonalization. Also, as stated previously, both integrals of any loop type that contains two integrals can be described by the same two coefficients $(x=0, x=1)$ in the region where the generators of a generator product overlap.

When two Gelfand states differ by two orbitals there will be at most one contributing loop to the matrix element of the states. The vast majority of nonzero matrix elements fit into this category. loops on the other hand can contribute to a great number of matrix 


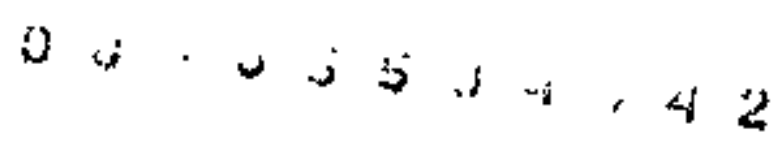

elements (but the average number of contributions per $100 \mathrm{p}$ is about two for the reported test calculations). Thus a Haniltonian stored in this manner is rore compact than the conventional form of CI matrix storage.

Once the loop type structure has been chosen, this information must be transferred into a form that can be used by the program. The loop searching naster tabie (Table IV) contains all of this information. Each loop is given four indices $i, j, k$, and $l$, where $i \leqslant f \leqslant k \leqslant i$. For loop type 1 all four indices are different and for loop type 14 all four are the same. Each loop starts at its lop leve1 $i$ and ends at its bottom level i-I. Any levels outside of this range have no effect on the generator coefficient values or integrals of the loop but would only affect the upper or lower walks of that loop and hence only affect which natrix elerents a given loop will contribute to. The value of any loop depends only on the shape of that loop and the starting spin value $\left(b_{q}\right)$ of the loop head,

Each loop type is broken into at most four sections depending on the number of differing indices. Loop types 1-7 all behave 1dentically within the $\ell$ to $k$ section. When generating these loops the work required for this section can be perforned once and used for any of the loops of these types. If each loop type was processed separately a sevenfold repetition of work would result in this section. Loop types that behave identically between the $t$ to $\mathrm{j}$ sections are:

1,2 , (and 7 if new loop types are used)

3 and 6

8 and 12

9, 10, and 13 . 
Loop types $\$ 1$ and 14 must be processed seperately since they have no sections in comon with any other loop types. Loop types that behave identically in the $j$ to $i$ section cannot be processed together but the same section of code can be used for each.

The use of loop types as a linear combination of integrals and overlaying then are essential features of the efficient implementation of the 1oop-driven algorithm. 


\section{E. The Loop-driven Methodology}

The loop-driven algorithn is without question the most important element accomting for the success of our GUGA nethod. The term "loop-driven algorith" is derived from the manner in which loops are generated. In a conventional CI (configuration driven), the next configuration on the configuration list determines what is done subsequently. With an integral driven approach it is the next integral on the list of integrals that determines what is processed naxt. In contrast to both of these approaches, with the loop-driven algorithm, the next loop that can be produced with the least effort is generated. Since most lops have large sections in conmon with many other loops, the savings are found in that the algorithm only generates portions of the next loop that differ from the previous loop. Generating loops in this ananer corresponds to neither a configuration driven approach nor an integral driven approach. Not only does this method illuminate vast numbers of previously unappreciated relationships between otherwise distinct Hamitonian matrix - lements, it also points out the relationships between different integrais that share one or more common indtces.

Figure 4 shows four sample two-electron loops. Once the first loop (solid lines) has been generated, the remaining three loops can be generated by simply finding and processing the segment shapes depicted by broken lines, usually with little additional effort. The four loops depicted also use different integrals that have iittle in combon with one another except some comon indices. Because of this, we clain that the integrats are processed simultaneously. While 
generating the loops for one integral, major portions of loops for other integrals are also being produced. The method used to find the loops is based on an exhaustive tree search wethod, 51 allowing a systenatic generation of all the loops for a given block of integrals. A block of integrais usually contains a11 two- and oneelectron integrals that share a common largest 1ndex. At each intermediate level all valid lower segment shapes are investigated. If the accepted segment represents a valid closure, then that loop is processed and the search continues at the previous level. If at any level a11 segment shapes have been exhausted, then the search a1so reverts to the previous level. In order to generate all of the loops, this tree search must be processed for every posstble loop head (each row of the DRT). The order in which distinct rows are processed in this manner is determined by the structure of the integral sorting arrays (see section III G.).

To process the above described tree search, pushdown stacks 51 are used. A pushdown stack is simply an array that spans the levels of the DRT. The use of these stacks is needed so that the progran can "remenber" where it left off at any level of the tree search when it returns to that level. Two of these stacks are coefficient stacks which are used to find the generator coefficients. Two are indexing stacks which are used to determine to which matrix elenents the generated $100 \mathrm{p}$ will contribute. Two of these stacks point to distinct rows of the DRT and are used to determine whether any given path to the next level is valid. An additional two stacks are segnent stacks which determine the loop type section, starting segment shape, 


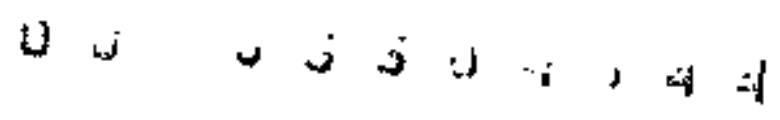

and the segment shape that was last tested. The last is a tracking stack used to determine the final offsets (in values) of the integrals to be used in deternining the loop value. Integral offsets are only updated whenever a $k, j$, or $i$ value is reached; thus the offsets are not needed to be stored in pushdown stacks.

To minimize the searching, the different loop types are overlayed in all areas where they behave identically (as deseribed in section III D.). Without this essential overlaying, the GUGA pethod would be at least a factor of two slower than it is in its present form. This is another manner in which the loop-driven algorithm provides insight into the Hamiltonian. Much additional unnecessary searching can be eliminated by recognizing at an early stage what segment shapes will not lead to valid loops despite the appearance of validity. In the first implenentation such tests were primarily used with regards to the total symmetry structure of the loop (the $m$ and $m$ ' branches of the loop must not differ by symmetry when the loop tail is reached). Since then, we have further reduced the anount of searching for valid segment shapes. Use of the new segment coefficients does not allow loops to end with both $n$ and $m^{\prime}$ branches having an $s$ value of zero at the bottom segment (the last orbital unoccupied in both Gelfand states). Thus, when there are no electrons remaining in all of the lower orbitals, there is no need to test any further segments along this line. Another reason that the anount of searching has been reduced is that by using the new segment coefficients, loop types 1 , 2 , and 7 now behave 1dentically in the loop region between $\mathrm{j}$ and $\mathrm{l}$. In the prelialnary 1mplementation, they only coincided in the region 
from $k$ to $l$. Since these types include the rost comon loops, the reduction of repetition in this area has led to improved tinings, both because fewer segment shapes are searched for and because fewer segments are accepted and processed.

The loop-driven algorithn is a completely general method and it will efficiently generate all of the loops for any distinct row table (DRT), regardless of the complexity and the level of excitations included in the DRT. 


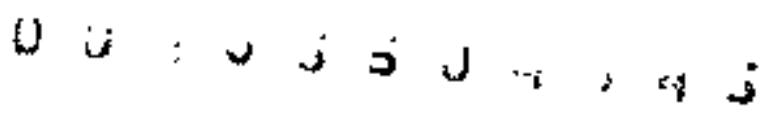

\section{F. The Loop Searching Master Table}

The loop searching master table (Table IV) contains all of the information about loop types and how they are overlayed. Within the loop-driven formalism, tt controls the path of the tree searching algorithm. In one sense this information completely defines the nature of the Hamiltonian and the DRT is only used as a template or backbone on which loops are generated. This infornation is in a similar forr to that needed by an efficient implementation of this method. (In fact a listing of a working progran using this nethod was consulted to produce this table.) Th1s table is also an invaluable tool to anyone attempting to create or modify a progran using the loop-driven algorithr.

The ISEG value refers to a particular searching section. For a given ISEG value all JSEG values within its range wi1l be investigated at that level within the DRT before reverting to a previous higher 1eve1. For example, when ISEG=3, JSEG values from 35 to 52 are tested. Each ISEG value corresponds to a particular segment type. Not only Is the shape of the segnent determined but also its range and position within possible valid loops. The search is always intiated at the loop head where ISEG=1.

The columns labeled IS( $\Delta b)$, by, and FS( $\Delta b)$ uriquely determine the shape of the seginent. These colunns are not needed by the searching algorithm but are included here to make the table more readable. The heading IS refers to the "initial shape" (top of segpent) and the heading FS refers to the "final shape" (bottom of segment); each identified by one or two letters. In this notation $R$ represents a 
raising generator and $L$ represents a lowering generator between the $n$ and in' branches of the loop at that intermediate level. A zero implies that the $\mathbf{n}$ and $n$ ' branches of the loop coincide at that intermediate level (a necessary condition for the loop head or tail). The segment shape is further specified by $a b^{b}$ value where $a b=b-b^{\prime}$. Whenever an IS or FS tern contains a $" /{ }^{n}$ this implies that the segment shape may be used in a loop type where a linear combination of integrals is reeded. When a compound integral track (two values) is present, the integral defined by the second track value often corresponds to the shape factor to the left of the "/". Whereas the IS and FS colurns refer to the starting and ending shape, the "by" columns define the body of the 100 p segment. The letters $R$ and $L$ again refer to raising and lowering genergtors respectively. A bar over or under a letter indicates the initiation or termination of a generator (top or bottom segment of the generator range) respectively. The letter $W$ indicates that a weight generator for this level is present. An asterisk on any generator simply means that a change of sign is introduced to the $x=1$ branch of the $100 p$ coefficient. Further details and use of this notation can be found elsewhere. 46

The coltun labeled "next" specifies the next ISEG block that Just be tested at the following lower level if the current segment at the present level is gccepted. A "next" value of zero implies that this segment if accepted will terminate a loop and that this loop should then be processed before continuing further with the search. The $s^{\prime}$ and $s$ values simply identify the case numbers for the $n^{*}$ and n branches of the $100 \mathrm{p}$. These values are compared with the arcs of 
the Shavitt graph at the current position to determine if the current segment is acceptable. The column labeled "tracks" gives the final offset values used in detemining the position of integrals within the current integral block. When a loop is terminated the current track value(s) is used when processing that 1oop. Wherever a segment is accepted where the track value is not specified, the track value(s) from the previous level is used as the current tracks value. A track value with only a single integer $\left(m_{1}\right)$ implies that only one integral will contribute to the loop and that the $X^{\prime}$ coefficient will be used to deterine the coefficient of that integral. For this case the loop value (v) is given by:

$$
r=X^{\prime} I\left(a^{r}+m_{1}\right)
$$

where 1 represents the array containing the current block of integrals and $a^{\prime}$ is the address offset within that block determined by $(i, j, k$, and $\ell$ ] and is given by:

$$
a^{\prime}=K_{k \ell}+J_{j}\left(\Gamma_{k} \times \Gamma_{\ell}\right)+I_{i}\left(\Gamma_{j} \times \Gamma_{k} \times \Gamma_{\ell}\right)
$$

where $K, J$, and $r$ are the integral offset arrays (see section III G.). When the tracks value contains two integers $\left(m_{1}, m_{2}\right)$ the $100 p$ value is given by:

$$
V=X^{\prime}\left[I\left(a^{\prime}+n_{1}\right)+Z^{\prime} I\left(z^{\prime}+m_{2}\right)\right] .
$$

When the tracks value contains two or three integers $\left(m_{1}, n_{2}, n_{3}\right)$ enclosed in parentheses only the $x^{\prime}$ value is used as the coefficient for all these integrals which implies that the loop value for three integrals can be expressed as: 


$$
v=X^{\prime}\left[I\left(a^{r}+m_{1}\right)+I\left(a^{\prime}+m_{2}\right)+I\left(a^{\prime}+m_{3}\right)\right]
$$

The column labeled "kjcond" contains information used to determine if a "k" or "j" value has been reached at the current level. When a "k" or "j" level has been reached its contribution to the integral address offset is determined. A k-condition of $-1,0$, and 1 inply the $\mathrm{k}$ level has not yet been reached, (the current segment is above the $k$ segment), the $k$ segnent has already been reached (the current $s$ egment is below the $k$ segment), and that the current level is the $k$ level segment, respectively. The distinction between $k$-condition values of $-I$ and $\theta$ is needed to test whether or not a block of integrals sharing a largest common index has been divided into subgroups (due to internal starage limitations for large calculations). The $j$-condition can have only two values, 1 and 0 . A value of 1 implies that the current level is the $j$ level segment. It should also be pointed out that the $f$ level segment camnot be above the $k$ level segment and that a valid loop may not terminate unless both the $j$ and $k$ level segments have been reached.

As stated previously (section II C.) the coefficient of any twoelectron integral in a particular loop can be represented by:

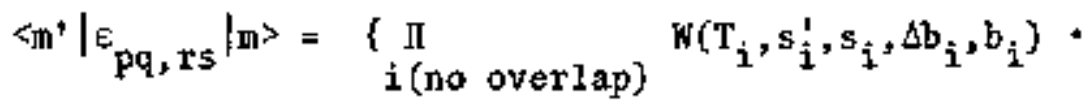

$$
\begin{aligned}
& \sum_{x=0,1} \underset{i \text { (overlap) }}{W\left(T_{i}, s_{i}^{\prime}, s_{i}, \Delta b_{i}, b_{i}, x\right)},
\end{aligned}
$$

where there is a different set of $\mathrm{W}$ coefficients for any loop segnent. These coefficlents are stored in equations under the heading of 
"coefficients". Within these equations there are two pushdown stacks ${ }^{51}(X$ and $Y$ ) and a third simple variable $Z$ '. The pushdoum stacks used here can sinply be thought of as arrays that span the levels of the DRT. During loop generation, when segment is accepted the composite contribution of all upper segments ( $X$ and/or $Y$ ) are updated with the contribution of the current loop segment, and the new conposite contribution is stored at the current level $\left(X^{\prime}\right.$ and/or $\left.Y^{\prime}\right)$. The $X$ stack is always used at every level and the $Y$ stack is only used for the $x=1$ term when both $x=0$ and $x=1$ terms are present. The $Z^{\prime}$ elenent is occastonatly changed. The segment coefficients are expressed in terms of the initial $b$ value of the $m$ branch of the loop using the definitions:

$$
\begin{aligned}
t & =1 / \sqrt{2} \\
A(p, q) & =\sqrt{\frac{b+p}{b+q}} \\
B(p) & =\sqrt{\frac{(b+p)(b+p+1)}{2}} \\
C(p) & =\sqrt{\frac{(b+p-1)(b+p+1)}{b+p}} \\
D(p) & =\sqrt{\frac{(b+p-1)(b+p+2)}{(b+p)(b+p+1)}} \\
E(p) & =\sqrt{\frac{2}{(b+p)(b+p+2)}} .
\end{aligned}
$$

The coefficlent codes listed in the 1ast column sinply assign an arbitrary number to each unique set of coefficient equations. This allows an easy and compact storage of the coefficient information through the use of a large "computed 50 To" within the loop generation al gorithn. 
It should be mentioned that the choice of segment coefficient and overall structure of this table is not umique, and a large number of decisions were made in its preparation. Nost of the credit in determining the segment coefficients presented here goes to Paldus ${ }^{45}$ and Shavitt. ${ }^{46}$ When some of the newer developments are Included such as repartitioning the Haniltonian relative to a "reference state" instead of relative to the "vacuun" as described by Shavitt, ${ }^{46}$ the $100 p$ searching master table must be expanded and adapted to allow for this additional corplexity. One major advantege of presenting the Hamitonian in this manner is that when a change of this nature is required, the loop generation algorithn need not be altered. It is sufficient to change only the information stored in the table. 
U : :

\section{Integral Sorting and Storage}

There are three reasons for resorting ${ }^{52}$ the one- and two-electron integrals for the GUGA method. The most important reasons is that nany loops require two or three integrals to deternine the loop value, and these different integrals must be in core at the sane time. Even better, if the different integrals needed were stored in adjacent positions, then only one address pointer would be required for any loop. Since loops are generated by the tree search method, all integrals used for any given tree search will have a largest integral index of $\ell$, the level of the loop head, It is therefore highiy advantageous to hold all integrals of a given largest index in core at any one tine, and they should all belong to the sane integral block if possible. Another reason for sorting the integrals is that once they are sorted, they can be stored in the nanner presented here. For some cases this implicit addressing storage method requires only half of the storage space required wth a conventional integral tape that contains a label and value for every integral. The last reasons mentioned here is that since a reordering of the orbitals is almost a necessity for the GUGA method, the integrals must undergo an indices change, unless the orbitals are reordered before the integral transformation. The transformation algorithn developed for use with GUGA CI applications creates and stores integrals in this manner thus eliminating an explicit sorting step later with the additional benefit of reduced externa1 storage requirements.

The integral sorting could bo avoided with an integral driven nethod, but as stazed previously this type of approach would result 
in a factor of 2 increase in the size of the fornula and diaganalization tapes. The integral oriven approach would also result in a manyfold repetition of work in the 1oop generation steps. Another way of understanding this difference is that the integral driven approach treats each integral separately whereas the loop-driven algorithen processes a block of integrals simultaneously. For these reasons this approach would be at best an order of magnitude less effective than the loop-driven algorithm, (unless loops are generated implicitly).

For a given loop head all integrals that could be required are stored, if possible, in the sane block. If there are more of these integrals than the block size, then these integrals are divided into different blocks in such a way that the repetition of work is nininized. The integral blocks are defined in such a way as to minimize the number of blocks required for a given available core with the above restriction,

Each integral is assigned a block and a position as follows:

(1) Each integral is given a type $(t, t=1-7)$ and a subtype (m, m=1-3) and an $i, j, k$, and $\ell$ value where $i \leqslant j \leqslant k \leqslant \ell$.

The possible types and subtypes are: 


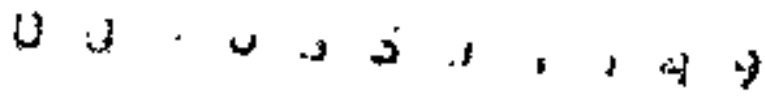

\begin{tabular}{|c|c|c|c|}
\hline$t$ & m & Integral & Condition \\
\hline \multirow[t]{3}{*}{1} & 1 & {$[\mathbf{i k} ; j \ell]$} & $i<j<k<l$ \\
\hline & 2 & {$[i j ; k \ell]$} & \\
\hline & 3 & {$[j k ; i \ell]$} & \\
\hline \multirow[t]{3}{*}{2} & 1 & {$[\mathbf{i j} ; \mathbf{j} \ell]$} & $i<j=k<\ell$ \\
\hline & 2 & {$[j j ; i l \ell]$} & \\
\hline & 3 & Not used & \\
\hline \multirow[t]{2}{*}{3} & 1 & {$[\mathbf{i k} ; \mathbf{i l i}]$} & $i=j<k<l$ \\
\hline & 2 & {$[i i ; k l]$} & \\
\hline \multirow[t]{3}{*}{4} & 1 & {$[i \ell ; j \ell]$} & $i<j<k=?$ \\
\hline & 2 & {$[\mathbf{i j} ; \ell \ell\}$} & \\
\hline & 3 & Not used & \\
\hline \multirow[t]{3}{*}{5} & 1 & {$[i l ; l l l]$} & $i<j=k=\ell$ \\
\hline & 2 & {$[\mathbf{i 1} ; \mathbf{i l}]$} & \\
\hline & 3 & $\langle i|\hat{\mathrm{h}}| \ell\rangle+$ (frozen core) & \\
\hline \multirow[t]{2}{*}{6} & 1 & {$[1 \ell ; i l]$} & $i=j<k=\ell$ \\
\hline & 2 & {$[i i ; \ell \ell]$} & \\
\hline \multirow[t]{2}{*}{7} & 1 & {$[\ell \ell ; l l l]$} & $\mathrm{i}=\mathbf{j}=\mathrm{k}=\mathrm{l}$ \\
\hline & 2 & $\langle\ell|\hat{\mathbf{h}}| \ell\rangle+($ frozen core $)$ & \\
\hline
\end{tabular}

The positions recorded as "not used" are kept as place holders (in cases ifj) so that the addressing can be defined simply by arrays. For large calculations these holders should only increase the integral space required by about $4 \%$. Integrals that correspond to frozen core or deleted virtual orbitals are not stored. There is also no space held for integrals that are zero by symmetry. 
(2) Given an $i, j, k, 2$, and $\mathrm{m}$ value, the address a within the proper block (determined by $k$ and $\ell$ ) is given by:

$$
a=K_{k \ell}+J_{j}\left(\Gamma_{k} \times \Gamma_{\ell}\right)+I_{i}\left(\Gamma_{j} \times \Gamma_{k} \times \Gamma_{\ell}\right)+m,
$$

where $\Gamma_{i}$ is the trreducible representation of orbital i. $K, J$, and $I$ are offset arrays generated based on the symmetry of each orbital and the number of orbitals. The integral addresses are generated during the loop generation. As $k, j$, and $i$ values are reached, the integral offsets are updated. This minimizes the amount of computational effort required in generating integral addresses. The $m$ values for any given loop are determined by the tracking pushdown stack.

Within this framework entire blocks of integrals are processed together; that is, all possible loops requiring these integrals are generated sequentially. This is also used very efficiently as a direct CI method ${ }^{7}$ where the storage of the diagonalization tape is avoided. 


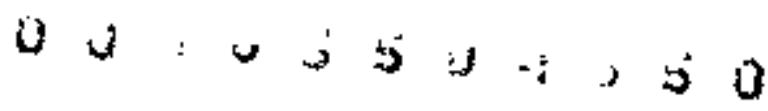

H. The Loop Breakdown Algorithm (Solution of the Upper Halk Problen)

In the early stages of the development of the GUGA CI method one of the najor stunbling blocks was that there was no simple way to easily find all of the Hamiltonian contributions of a given loop once it had been generated. Due to the nature of lexical ordering the lower walks of a loop were sequential, thus easily detemined. This problent was then reduced to the task of finding all of the upper walks for a given loop ("the upper walk problentr). At that tine there were several proposed ntethods to solve or circumvent this problem but of these all had serious drawbacks. One solution that would have been prohibitive in terms of computational efficiency, was to simp1y search for all upper walks whenever a loop was generated. Another solution with a similar deficiency would be to regenerate a given loop at different times for each upper walk. The only solution which appeared to be acceptable was to consult a list of upper walks from the loop head of each generated loop, but this approach was discarded in favor of the more efficient loop breakdown algorithn presented here.

Due to the nature of the lexical ordering ${ }^{24}$ of configurations (wa1ks), a11 configurations that pass through a given distinct row with a given upper walk are contiguous. All of these configurations can be simply referenced by a starting position and a length.

$$
\mathbf{m}=\mathbf{m}_{\mathbf{T}}+\mathrm{k}=1,2, \ldots, \mathrm{x}_{\mathbf{r}},
$$

where $m_{r}$ is the weight of the given upper walk and $x_{r}$ is the number of lower walks of that row. Whereas the lexical ordering of states greatly facilitates finding configurations of lower walks through any 
row, this is not so for upper walks. The upper walk problen is to find an effective algorithin that can generate all the upper walks for any loop. Consider a different ordering of states where all configurations passing through a given distinct row with a given lower walk are contiguous or, in other words, all upper walks are sequential. This ordering of states would be roughly equivalent to the lexical ordering of states if the DRT was inverted. This ordering of states, termed the "reverse lexical order," is related to the lexical ordering of states by the indexing array $Y$, such that

$$
\overline{\mathbf{n}}=\mathrm{Y}(\mathrm{m})
$$

where $\bar{m}$ is the reverse lexical order index and $m$ is the lexical order index of a given state. Each distinct row is also assigned a primary upper walk welght, ${ }^{2}$, and the number of upper walks, $\bar{x}_{r}$, as well as the number of lower walks, $x_{r^{*}}$ All states that pass through a given row are given by:

$$
\begin{aligned}
\mathrm{m}=\mathrm{Y}\left(z_{\mathrm{r}}+\mathrm{k}\right)+\mathrm{j} & =1,2,3, \ldots, \bar{x}_{\mathrm{r}} \\
\mathrm{k} & =1,2,3, \ldots, \mathrm{x}_{\mathrm{r}} .
\end{aligned}
$$

Here the final states are in reverse lexical order. To extend the use of the 1ndexing array to loops, all watrix element contributions $H_{\bar{n}, \mathrm{n}}$, can be found simply by

$$
\begin{array}{ll}
\overline{\mathrm{i}}=\mathrm{Y}\left(\mathrm{z}_{\mathrm{h}}+\mathrm{m}_{\ell}+\mathrm{k}\right)+\mathrm{j} & \mathrm{j}=1,2,3, \ldots, \bar{x}_{\mathrm{h}} \\
\overline{\mathrm{m}}{ }^{\prime}=\mathrm{Y}\left(\mathrm{z}_{\mathrm{h}}+\mathrm{m}_{\ell}+\mathrm{k}\right)+\mathrm{j} & \mathrm{k}=1,2,3, \ldots, \mathrm{x}_{\mathrm{t}},
\end{array}
$$


where $\mathbb{m}_{2}$ and $\mathrm{m}_{2}$ are the weight of each branch of the loop and the subscripts $b$ and $t$ refer to the loop head and tail rows, respectively. Agatn the final indices are in reverse lexical order. A given loop will contribute to the Hamiltonian $x_{t} \cdot \bar{x}_{h}$ times. This breakdown algorithn is needed in the diagonalization step and in the generation of the one- and two-particle density matrices, strce the information required for this algorithm can be stored tn a compact form with only three quantities needed:
1) $z_{h}+m_{\ell}$
2) $z_{h}+m_{l}^{\prime}$
3) $x_{t}$

Here $\bar{x}_{h}$ is not present because it is best stored as a flag since its value is constant for all loops from a given loop head. The final loop value Hust also be written as a real number.

Due to the nature of the indexing array $\gamma$ all contributions from any given loop will lie on the same super-diagonal (i,e, $\bar{n}-\bar{n}$, = $Y\left(z_{h}+w_{l}\right)-Y\left(z_{h}+n_{l}\right)$, which is constant $)$. This 10op breakdown algorithm allows a rapid determination of all matrix element contributions and its only drawback is that an integer indexing array that exactly spans the configuration basis must be held in core during the diagonalization. 


\section{ImpIicit Treatment of the External Space}

For a theoretical study in which only single and double excitatlons into the external space are allowed, it is possible to generate loops that are rostly or wholly within this space in a manner that is more efficient than the loop-driven algorith, When the external orbitals are placed at the botton of the DRT, the Shavitt graph is quite simple for reasons described earlier. At any external leve1 there can be (ignoring symmetry distinctions) at most four rows, designated $W$ (two electrons singlet coupled in lower orbitals), $x$ (two electrons triplet coupled in lower oxbitals), Y (one electron in lower orbitals), and $z$ (no electrons in lower orbitals). Because of this simple structure a11 loop shapes and coefficients in this area can be implemented implicitly. This idea was first suggested by siegbahn ${ }^{53}$ for an integral driven approach. of course the generation of loops for a given integral on denand by using the factorization of coefficients over levels is a slow and repetitious process. Thus the only hope for an integra1 driven approach is to generate the vast majority of loops in an implicit manner. This idea was carried further by shavitt, ${ }^{54}$ who worked out the shapes and details for all of the different loop types with the virtual orbitals correctly placed at the bottom of the DRT. We have implemented the generation of the all-external implicit loops in a manner simflar to the method suggested by Shavitt but with a few modifications. The method used is somethat analogous to the loop-driven algorithn, in that we proceed by bringing in a block of integrals (abc) for a given largest integral index (d) stored in the manner described in section III $G$. Once this is done one enters 


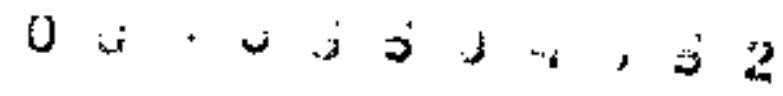

a set of three nested DO loops over the remaining indices (abc) where the number of operations in the inner loops has been nininized so that any symnetry pointer or offset that is used for different integrals is computed only once. In the next to innermost Do loop where the indices (bcd) have been established, the Ioops are essentially deterninted. The final offset is added and the GugA loops are processed in the innermost do loop. Also, in this formulation it is easy to treat those integrals with coefficients of $\pm l$ in a more efficient manner by avoiding unnecessary multiplications.

We find that the inplicit generation of loops is almost a factor of two faster than the loop-driven algorithm for the all-external space. For the partly external loops this ratio will not be as large as two-to-one. For Example $\mathrm{I}\left(\mathrm{BH}_{3}, 34\right.$ orbitals in $\mathrm{C}_{2 \mathrm{~V}}$ sugbroup) the loop-driven algorthm required 23.0 seconds to generate the allexternal loops and the implicit generation required 13.4 seconds to generate the same loops within the 30 virtual orbitals. The tine required for the remaining loops was 125.7 seconds, giving a total savings of $7 \%$ (neglecting time required for DRT generation and integral sorting) by the inplementation of the implicit generation of all-external loops when the ratio of external to intermal orbitals is 7.5 to 1 . When the implicit generation is also put into practice for loops with two or three external indices, the overall savings will be perhaps 30\% compared with the purely loop-driven algorithr. For Example II $\left(\mathrm{Ch}_{2}, 61\right.$ orbitals $\mathrm{C}_{2 \mathrm{~V}}$ point group) where the ratio of external to internal orbitals is 11 to I the percent savings in the loop generation phase is found to be $9.4 \%$ using the inplicit generation of the all-external loops. 
J. Diagona1ization Tape vs, Direct Method

As shown earliex, the GUGA method is quite sultable as a formala tape method, albeit the formula tape generation step is so fast that it may not be worth saving from one calculation to the next. In this section we discuss the calculations in which the formula tape generation 1s omitted and the diagonalization tape (symbolic formulas a1ready combined with molecular integrals) is generated directly. Since no sorting is required, it is also possible to implewent the loop-driven algorithm as a direct mothod, ${ }^{7}$ in which all of the loops are generated during each iteration of the diagonalization without losing any efficiency in terms of the slmultaneous treatment of integrals. For Example I $\left(\mathrm{BH}_{3}, 34\right.$ orbitals, $\mathrm{C}_{2 \mathrm{v}}$ subgroup) the conputation tine required to generate and process all of the loops (neglecting DRT generation, buffer packing and integral sorting) is 122.9 seconds for the loop-driven algorithm. The time required for the matrix multiplication $\sigma_{i}=\sum_{j} H_{i j} c_{j}$ at each iteration of the diagonalization is 32.2 seconds for an overall ratio of 3.8 to 1 . It should be mentioned again that, because of the nature of the loop-driven algorithm, each nonzero $H_{i j}$ has (on the average) just over one contributing loop, because loops are linear combinations of integrals, whereas for an integral driven method there will be almost two contributions per nonzero $H_{i j}$. Thus, the iteration time required for the multiplications in $\sigma_{i}=\sum_{j} H_{i j} c_{j}$ will be almost twice as large for an integral driven method.

The ratio of loop generation time to diagonalization iteration time is important because st will become cost effective to generate all of 


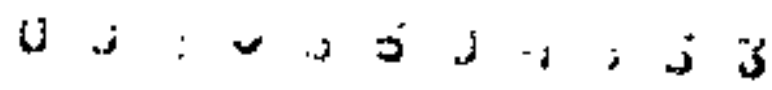

the loops each iteration of the diagonalization when this ratio becomes suall enough. For each machine the break-even point will depend on the particular charging algorithn used to calculate the cost. With the advantages of treating the all-external space inplicitly the ratio reduces to 3.5 to 1 . When the parts of other loops with three and two external indices are treated inplicitly, the savings nay be perhaps $30 \%$ over the purely loop-driven algorithm, and with that improvement the ratio will be reduced to 2.7 to 1 . It is our contention that further inprovenents elsewhere can bring this ratio to 2 to 1; that is, it will take only twice as long to create the complete faniltonian matrix as it does to multiply it by a vector. Even if this approach is not cost effective, for very large calculations, the external storage requirenent of the diagonalization tape may make the direct method preferable. Although many of our algorithms have been written with the direct method in mind, we cannot Implement this on our Harris niniconputer ${ }^{55}$ for any reasonably sized calculation because of severe internal (core) storage requirements. 


\section{K. Large Matrix Diagonalization Method}

For the GUGA method, a straightforward diagonalszation using the method of Davidson ${ }^{56}$ requires that two real vectors and one integer vector spanning the configuration basis, and the buffer space reside in core simultaneously. On a minicomputer such as ours ${ }^{19}$ (with only 20k of real woxds available to the user) this limits the size of the secular equation to at most 8000 configurations. By today's standards this is not considered to be large, and nary problems of current interest require considerably bore configurations than this. 57 To develop a nethod for ninicomputers that will allow very large matrices to be diagonalized, it is elear that the matrix nust be partitioned. One method of doing this is to read the entire Hamiltontan several times, using only the portions that correspond to the particular section that is currently being processed. However this rapidly becones prohibitive when the vector is broken up into $n$ sections if $n$ is more than two, since the number of passes is given by $n(n+1) / 2$. Clearly what is needed is to sort the matrix into $n(n+1) / 2$ sections, each of which can be read when the proper portion of each vector is in core. If $\mathrm{n}$ ts minimized in such a way that only two portions of vectors are needed at one time, most of the Haniltonian will need to be read and processed twice, since only the lower half of the Haniltonian is stored. Taking another approach, doubling the size of $n$ implies that each portion of the CI vector is half as large so that four portions can be held in core at once. This allows the step $g=$ Hc to be carried out by reading the matrix only once and processing a given $H_{i j}$ twice when $i \neq j$ (the only drawback befng an 
increased number of Hamiltonian sections\}.

Since $20 \mathrm{~K}$ real words are available to the user, after leaving some space for buffers each portion of the vector can at nost be about 4000 configurations. A rather fortuitous coincidence is that the Harris Slash Four word size is 24 bits. Half of a word is thus 12 bits, which can be used to specify an address fron 1 to 4096. Thus by choosing 4096 as our block size we also can specify a given element ( $I$ and $J$ ) with a single word.

Another fortuitous accurrence involves the use of the GUGA in reverse lexical ordering as described in the loop breakdown section. The average loop has more upper walks than lower walks, and each contribution for different upper walks can be specified by a starting matrix element and a length, which define other natrix elenent contributions along the given super-diagonal. These contributions will usually Ije sequentially within the same Hamiltonian block. The cortbination of storing $I$ and $J$ addresses in a single word and using reverse lexical ordering allows us to store an entire Hamiltonian in about half the space required for the corresponding conventional natrix. Since this method is limited onty by available disc space (up to about 30,000 configurations) this essential1y doubtes our capabilities.

The final "matrix" tape is also sma1ler than the unsorted diagonalization tape, so the Yoshimine sorting nethod ${ }^{58}$ we use allows the final matrix tape to be stored in the same external space that the original unsorted diagonalization tape is read from.

One of the largest variational wavefunctions determined stemined from a three reference interacting space treatment (two closed shell 
singlets, one open shell singlet) of $\mathrm{SO}_{2}$ that contains 23,613 configurations. This calculation required about 60 megabytes of external storage, or less than half what is available to any user on our system. Thus calculations on the order of 25,000 will become routine on our minicomputer. For this calculation, the diagonalization tape was generated in only 39 minutes at a real cost of about $\$ 5$. The time required to sort this matrix is 57 minutes; however, this sorting would not be necessary if enough core storage were available. Each iteration of the diagonalization step required 19 minutes and a total of 9 iterations were required to obtain the second root only. The diagonalization time is between 106 and $20 \%$ longer than it would be on a similar computer that could store and process two complete real vectors in care. When tncluding the sorting tine as well as the reduced efficiency, we find that the present diagonalization procedure increases the (unlinited central memory) diagonalization time for any given calculation by about 50s, Since the diagonalization step is not usually the nost time-consuming portion of a single calculation, we do not find this to be a major drawback. 
$0, i, j, j, j$

\section{Density Hatrix Analysis}

We have also developed a one-particle density matrix analysis which enables us to obtain one-electron properties at the CI level. Unlike conventional CI methods, where the optimum method for generating the density natrix is to read and process the one-electron portion of the formula tape, we do not have a separate one-electron portion of the fornula tape, nor a configuration list. Instead we slmply generate a11 of the one-electron loops and use the loop breakdown algorithon to rapidiy find all of the CI vector coefficients. Ne find that the time required to generate the one-particle density matrix by this method is negligible compared with the other steps involved in the CI. Once the one-particle density matrix is created, it can be transformed to the $A O$ basis and then passed to a one-electron properties routine. 
M. Avoidance of the Full Integral Transformation

Previous work by Pople and coworkers ${ }^{38}$ describes a method that partly avoids the explicit ( ${ }^{5}$ ) four-index transformation of atonic integrals to a molecular basis. In particular, their work shows how the integrals [ab;cd] never need to be computed (where all of the indices $(a, b, c, d)$ correspond to the external space). Their method allows the contributions of the all-external integrals to be included directly from the atomic integrals $[\lambda \mu ; v \sigma]$, usually with a reduction of necessary operations. This approach has some similarities to the self-consistent electron pair (SCEP) method of Meyer ${ }^{59}$ and bykstra et al. ${ }^{60}$

One of the drawbacks to Pople's Eormalism is that it is only applicable to CI methods that include up to double replacenents from a single reference slater determinant. We will show how this formal ism can be extended to a completely general CI procedure over spin-adapted Gelfand states of a multireference interacting basis, or any other set of Gelfand states where at nost two electrons are allowed into some external space.

Using Davidson's method for diagonalization, ${ }^{56}$ each iteration of a o vector must be found such that

$$
\sigma_{I}=\sum_{J} H_{I J} C_{J}
$$

The all-external two-electron integral contribution to the $\sigma$ vector can be expressed as

$$
\sigma_{I}^{e x t}=\sum_{J} \sum_{\lambda \xi \downarrow \sigma} C_{J} c_{\lambda a} c_{\mu b} c c^{c} c_{\sigma d}[\lambda \mu ; w] b_{I J}^{a b c d}
$$


0 i $v j 3+i, j 6$

where $C_{J}$ is the $C I$ coefficient of configuration $J$ and $[\lambda \mu$; $w]$ is an integral in the AD basis. The $\mathrm{b}_{\mathrm{IJ}}^{\text {abco }}$ term is simply a spin coupling coefficient where the indices $(a, b, c, d)$ are all restricted to the external orbitals. For a determinant based $C I$ the coupling coefficients $b_{\text {IJ }}^{\text {abcd }}$ will either be tuity or zero. On the ather hand, when a Gelfand configuration basis is used the coupling coefficients becone more complex. This additional complexity requires sone modification of the formalisn presented by Pople. 38

If two electrons occupy the external space, they can only be coupled in two ways (singlet or triplet coupled) if the ordering of orbitals is chosen in the manner described earlier (where all of the external orbitals are placed at the bottom of the Shavitt graph or DRT). If the electrons are triplet coupled, then this Gelfand state can only have a nonzero matrix contribution of the all-external integrals with other Gelfand states that also have two triplet coupled external electrons. These states are referred to as $X$ states and the sptn coupling coefficients are given by

$$
b_{I J}^{\text {abcd }}=b_{I_{u p}, J} \operatorname{sgn}[a-c] \operatorname{sgn}[b-d]
$$

where $I_{\text {up }}$ refers to a particular upper walk of configuration $I$ in the 1nternal space. The function $\operatorname{sgn}[x]$ is defined as

$$
\begin{aligned}
& \operatorname{sgn}[x]=1 \text { if } x>0 \\
& \operatorname{sgn}[0]=0 \\
& \operatorname{sgn}[x]=-1 \text { if } x<0
\end{aligned}
$$

For the $X$ states, the coefficients are already factored into an ac term 
and bd term.

States where the two external electrons are singlet coupled are referred to as states. The spin coupling coefficients for these states are given by

$$
\mathrm{b}_{\mathrm{IJ}}^{\mathrm{abcd}}=\delta_{\mathrm{I}_{\mathrm{up}}, \mathrm{J}_{\mathrm{up}}}\left[(1+\sqrt{2}-1) \delta_{\mathrm{ac}}\right)\left(1+(\sqrt{2}-1) \delta_{\mathrm{bd}}-\delta_{\mathrm{ac}} \delta_{\mathrm{bd}}\right] \text {. }
$$

For the $w$ states, it is not as easy to factor the spin coupling coefficient tnto ac and bd parts. By rewriting configuration $I$ as elther $u_{W_{W}}+$ ac or $u_{X_{X}}+a c$, equation $(68)$ can be rewritten as

$$
\begin{aligned}
& \sigma_{{u p_{W}+a c}^{e x t}=} \sum_{b d} \sum_{\lambda \mu v \sigma} c_{u p_{W}}+b d{ }_{\lambda a} c_{\mu b} c_{w c} c_{\sigma d}[\lambda \mu ; v \sigma] . \\
& {\left[\left(1+(\sqrt{2}-1) \delta_{a c}\right)\left(1+(\sqrt{2}-1) \delta_{b d}\right)-\delta_{a c} \delta_{b d}{ }^{3}\right.}
\end{aligned}
$$

and

$$
\begin{aligned}
& \sigma_{u p_{X}+a c}^{e x t}=\sum_{b d} \sum_{\lambda \mu \sigma \sigma} c_{u p_{X}+b d} c_{\lambda a} c c_{\mu b} c_{v c} c_{\sigma d}[\lambda \mu ; v \sigma] \\
& \operatorname{sgn}[a-c] \operatorname{sgn}[b-d] \quad
\end{aligned}
$$

Rather than first finding [ab;cd] as is usually done, these equations can be solved by first forming the intermediates $F_{W}^{\mu \sigma}, F_{W}^{\mu \sigma}$, and $F_{X}^{\mu \sigma}$. defined as

$$
\begin{aligned}
& F_{W}^{\mu \sigma}=\sum_{b>d}\left(c_{\mu b} c_{\sigma d}+c_{\mu d} c_{\sigma b}\right) c_{u p_{W} \rightarrow b d} \\
& F_{W}^{\mu \sigma}=\sum_{b} \sqrt{2} c_{\mu b} c_{\sigma d} c_{u p_{W} \rightarrow b b}
\end{aligned}
$$

and 


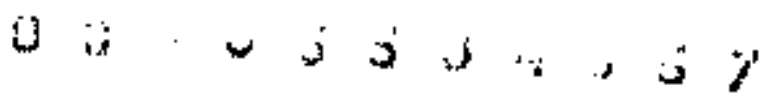

$$
F_{X}^{\mu \sigma}=\sum_{b>d}\left(c_{\mu b} c_{\sigma d}-c_{\mu d} c_{\sigma b}\right) c_{u p_{X}+b d} .
$$

The next step, which will be the most time-consuting, conbines the atomic integrals with these $F$ terms to form

$$
\begin{aligned}
& \tilde{\mathrm{F}}_{W}^{\lambda \nu}=\sum_{\mu \sigma} \mathrm{F}_{W}^{\mu \sigma}[\lambda \mu ; \omega \sigma] \\
& \tilde{\mathrm{F}}_{W}^{\lambda \nu,}=\sum_{\mu \sigma} F_{W}^{\mu \sigma},[\lambda \mu ; v \sigma]
\end{aligned}
$$

and

$$
\tilde{F}_{X}^{\lambda \nu}=\sum_{\mu \sigma} F_{X}^{\mu \sigma}[\lambda \mu ; \nu \sigma] .
$$

Using the $\tilde{\mathrm{f}}$ intermediates the $\sigma^{\text {ext }}$ contribution can easily be found as

$$
\begin{aligned}
\sigma_{u p_{X} \rightarrow a c}^{\mathrm{ext}}= & \sum_{\lambda v}\left[( \tilde { F } _ { X } ^ { \lambda v } + \tilde { F } _ { X } ^ { \lambda \nu \mu } ] \left(c_{\lambda a} c_{v e}+c_{\lambda c} c_{v a}+\right.\right. \\
& \left.(\sqrt{2}-2) c_{\lambda a} c_{v a} \delta_{a c}\right)-\frac{\sqrt{2}}{2} \tilde{F}_{X}^{\lambda \nu}{ }^{\lambda \nu}\left(c_{\lambda a} c_{v a} \delta_{a c}\right)
\end{aligned}
$$

and

$$
\sigma_{u p_{X} \rightarrow a c}^{e x t}=\sum_{\lambda v} \tilde{F}_{X}^{\lambda \nu}\left(c_{\lambda a} c_{\nu c}-c_{\lambda c} c_{\nu a}\right)
$$

As stated previously, the most time-constming step is described by equations (77-79) and it is of the order $\left(n^{2} n^{4}\right)$ where $n$ and $n$ are the number of internal orbitals and the number of basis functions respectively. The step that this avoids reduces the integral transformation from an $\left(\mathrm{m}^{5}\right)$ process to an $\left(\mathrm{nm}^{4}\right)$ process. Therefore, whether this type of approach is optimum for a given CI problem will depend on the relative comparison of $\mathrm{n}^{2}$ and $\mathrm{m}$ as well as other factors. It should 
also be remembered that the procedure presented here must be performed every iteration of the diagonalization. As it will be shown in a later section, the real advantage of this type of an approach will come about for analytic gradients of CI energies. 
U.

IV. ANALYTICAL GRADIENTS FOR CI ENERGIES

One of the nost pronising aspects of the GUGA formalism will be in the determination of analytical gradients (first derivatives) of configuration interaction energies with respect to changes in muclear positions.

The current conventional approach to calculating energy derivatives at the CI Ievel is through the use of the finite-difference method. This is done by performing several distinct calculations (at least two) at nearby geometries for each degree of freedom that is needed. The desired energy derivative for any perturbation can be approxinated by

$$
\frac{\partial E(x)}{\partial x} \approx \frac{E(x+\Delta x)-E(x)}{\partial x}
$$

Whereas the nethods required to obtain $\mathrm{CI}$ derivatives in this manner are much simpler than those required to obtain analytic CI gradients, there are several drawbacks to this approach. The nost obvious drawback is that it is an approximation. If the step size $\Delta x$ is too large, the contanination effects of higher derivatives could becone toportant. On the other hand, $\pm f$ the step size is too small, roundoff effects could doninate. The problem of roundoff errors at the CI Ievel are much more pronounced than those at the SCF level (due to the nature of the four-index transfornationj. Perhaps a nore serious drawback is that flnding energy derivatives in this manner can be quite costly. Usually three calculations are required to obtain any derivatives accurately, thus for any (non-linear) molecule the approxi- 
mate cost to find all of the derivatives can be expressed as

$$
(3 \mathrm{~N}-6) \times 3 \times \mathrm{I}_{1}
$$

where $I_{1}$ is the cost of a single calculation. Using the nethods presented here, the cost required to obtain the identical results would be

$$
I_{1}+I_{2}
$$

where $I_{2}$ is the cost to obtain all of the energy derivatives, once the CI calculation has been completed. Since (as it will be shown) that $I_{2}$ is approximately equal to $I_{1}$ for most cases, and for difficult cases $I_{2}$ could be twice as large as $I_{1}$, savings of an order of magnitude could easily be found for a molecule as large as $\mathrm{C}_{4} \mathrm{H}_{6}$ using analytic gradient methods.

In this section, the theory behind these new nethods will be presented, as well as some details and insight into the first implementation (which at the time of this writing is near completion). 
(1) :

\section{A. Theory of CI Energy Gradients}

The GUGA method will be extremely useful iл determining analytical gradients ${ }^{61}$ (first derivatives) of Cl energies with respect to changes in nuclear positions. It is clear that not only can this method be implenentedin such a way as to be nore efficient than performing several distinct CI calculations, but also in such as way as to be flexible enough to bo useful for a general large scale multireferente CI with any desired configuration set.

For any $\mathrm{CI}$ wavefunction

$$
\phi=\sum C_{I} \phi_{I}
$$

the energy can be written as

$$
E=\sum c_{I} c_{J}\left(a_{I J}^{i j j}\langle i|\hat{h}| j\rangle+b_{I J}^{i j k \ell}[i j ; k \ell]\right)
$$

The derivatives of the electronic energy can then be expressed as

$$
E^{a}=\frac{\partial E}{\partial x_{a}}=\sum c_{I} c_{j}\left[a_{I J}^{i j}\langle j|b| j\rangle^{a}+b_{I j}^{i j k l}[i j ; k l]^{a}\right),
$$

since the CI coefficients are variationally determined, and thus the formula for the gradient w1Il not involve their differentials. 61 In equation (87) $a_{I J}^{i j}$ and $b_{I J}^{i j k l}$ are simply spln coupling constants. ${ }^{63}$ The derivatives of the integrals are given by 


$$
\begin{aligned}
\langle i|\hat{b}| j\rangle^{a} & =\sum_{\lambda \mu} c_{\lambda i} c_{\mu j}\langle\lambda|\hat{b}| \mu\rangle^{a}+\sum_{\lambda \mu}\left(c_{\lambda i}^{a} c_{\mu j}+c_{\lambda i} c_{\mu j}^{a}\right)\langle\lambda|\hat{h}| \mu\rangle \\
& =\sum_{\lambda \mu} c_{\lambda i} c_{\mu j}\langle\lambda|\hat{h}| \mu\rangle^{a}+\sum_{r}\left(U_{r i}^{a}\langle r|\hat{h}| j\rangle+U_{r j}^{a}\langle i|\hat{h}| r\rangle\right)
\end{aligned}
$$

and by

$$
\begin{gathered}
{[i j ; k l]^{a}=\sum_{\lambda \mu \nu \sigma} c_{\lambda 1} c_{\mu j} c_{v k} c_{\sigma l}[\lambda \mu ; v \sigma]^{a}=\sum_{r}\left[u_{r i}^{a}[r j ; k l]+\right.} \\
\left.u_{r j}^{a}[i r ; k l]+u_{r k}^{a}[i j ; k l]+u_{r l}^{a}[j j ; k l]\right)
\end{gathered}
$$

where $u_{r i}^{a}$ denotes the first order changes in the nolecular orbitals. ${ }^{62}$ To compute the energy gradients in this nanner one needs, in addition to the tnformation required in the ordinary $C I$, the derivatives of all of the atomic integrals $\left\langle\lambda|\hat{\mathrm{h}}| \mu^{\mathrm{a}}\right.$ and $[\lambda \mu ; \psi \sigma]^{\mathrm{a}}$ as well as the $\mathrm{U}_{\mathrm{r} \text { I }}^{\mathrm{a}}$ coefficients.

It is true that the contribution of the atomlc integral derivatives $\left[\lambda \mu ; v_{0}\right]^{a}$ can be obtained simply by a four-index transfornation, out this would require a separate $\left(m^{5}\right)$ transformation process for each derivative under consideration. We will present two methods here to avoid this problem. The first method shows how an $\left(m^{5}\right)$ transformation may be performed once and used for all of the different derivatives and the second method (given in section IV C.) denonstrates that much of even this one remaining $\left(\pi^{5}\right)$ transformation can be avoided.

By combining equations (88) and (89) with equation (87) the energy gradient can be expressed in two texms as 
U.

$$
\begin{aligned}
& \mathrm{E}^{\mathrm{a} \cdot}=\sum_{\lambda \mu \nu \sigma} \sum_{i j k \ell} \sum_{I J} c_{I} c_{J^{b}} b_{I J}^{i j k \ell} c_{\lambda i} c_{\mu j} c_{\nu k} c_{\sigma \ell}[\lambda \mu ; \nu \sigma]^{a}+ \\
& \sum_{\lambda \mu} \sum_{i j} \sum_{I J} c_{I} C_{J} a_{I J}^{i j} c_{\lambda i} c_{\mu j}\langle\lambda|\hat{h}| \mu\rangle^{a}
\end{aligned}
$$

and

$$
\begin{aligned}
& E^{a \cdots}=\sum_{i j r} \sum_{I J} C_{I} C_{J} a_{I J}^{i j}\left\langle u_{r i}^{a}\langle r|\hat{h}| j\rangle+U_{r i}^{a}\langle 1|\hat{h}| r\rangle+\sum_{i j k \ell r} \sum_{I J}\right. \\
& \mathrm{C}_{1} \mathrm{C}_{\mathrm{J}} \mathrm{b}_{\mathrm{IJ}}^{\mathrm{ijk \ell}}\left(\mathrm{u}_{\mathrm{ri}}^{\mathrm{a}}[\mathrm{rj} ; \mathrm{kll}]+\mathrm{u}_{\mathrm{rj}}^{\mathrm{a}}[i \mathrm{ir} ; \mathrm{kl}]+\mathrm{v}_{\mathrm{rk}}^{\mathrm{a}}[i j ; \mathrm{rl}]+\mathrm{v}_{\mathrm{rl}}^{\mathrm{a}}[i j ; \mathrm{kr}]\right)
\end{aligned}
$$

where the total energy derivative is given by

$$
E^{a}=E^{a-}+E^{a}+
$$

The optimum method for evaluating these equations is by first computing the one- and two-particle dersity matrices ${ }^{64}$ given respectively by

$$
G_{i j k \ell}=\sum_{I J} C_{I} C_{J} b_{I J}^{i j k \ell}
$$

and

$$
Q_{i j}=\sum_{I J} C_{I} C_{J} a_{I j}^{i j}
$$

The coupling coefficient $b_{\mathbf{I}}^{\mathbf{i j k} \ell}$ and $a_{I J}^{i j}$ ean be found rapidly by using the 1oop-driven algorithm. The density matrices are used in two different ways to find both the gradient contributions of $\mathrm{E}^{\mathrm{a}}$, and $\mathrm{E}^{\mathrm{a}}$..

To find the contribution $\mathrm{E}^{\text {as }}$ given by equation $(90)$, the density matrices are transformed to the AO basis by 


$$
Q_{\lambda \mu}=\sum_{i j} c_{\lambda \mathbf{i}} c_{\mu j} Q_{i j}
$$

and by

$$
G_{\lambda \mu v \sigma}=\sum_{i j k \ell} c_{\lambda i} c^{c_{j j}} c_{v k} c_{\alpha \ell} G_{i j k \ell}
$$

This step will be of the order $\left(n^{5}\right)$ and is similar to the four-index transformation, except that the transformation here is fron the wo to the AO bas1s which requires less effort than the reverse process whenever orbitals are either deleted or treated as frozen core orbitals. By substituting equation (95) and (96) into (90), $\mathrm{E}^{\mathrm{a} \text {. }}$ reduces to

$$
E^{a}=\sum_{\lambda \mu \nu \sigma} G_{\lambda \mu \nu \sigma}[\lambda \mu ; \nu \sigma]^{a}+\sum_{\lambda \mu} Q_{\lambda \mu}\langle\lambda|\hat{h}| \mu\rangle^{a} .
$$

The transformation of the density matrices is processed only once and the result is used for all of the different derivatives. Also, the integrals $[\lambda \nu ; v \sigma]^{a}$ are never stored. As they are generated, its product with its corresponding density matrix element is imediately added to energy derivatives terms.

The derivative contribution $\mathrm{E}^{2,2}$ given by equation (91) is found by first generating the Lagrangian $x_{\text {ir }}$ given by

$$
X_{i r}=\sum_{j k \ell} 4 G_{i j k \ell}[r j ; k \ell]+\sum_{j} 2 Q_{i j}\langle r|\hat{h}| j\rangle
$$

which is determined by an $\left(\mathrm{m}^{5}\right)$ process that is performed once. The effort required for this step is about one fourth of the effort required in a four-index transformation. This Lagrangian is an extrenely 


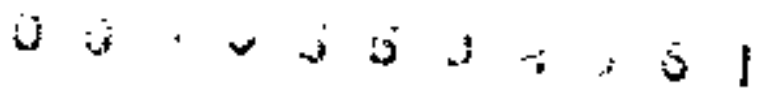

useful entity. When the density matrices are symetric, that is

$$
G_{i j k \ell}=G_{i j \ell k}=G_{j i k \ell}=G_{j i \ell k}=G_{k \ell i j}=G_{k \ell_{j i}}=G_{\ell k i j}=G_{\ell k j i}
$$

and

$$
Q_{i j}=Q_{j i}
$$

then the Lagrangian given by equation (98) can greatly simplify equation (91) and becomes

$$
E^{a \cdots}=\sum_{i r} x_{i r} u_{r i}^{a}
$$

which is processed when the $u_{r i}^{a}$ coefficients have been determined by the CPHF equations (see section IV F.). It should also be noted that this lagrangian $x_{i r}$ given by equation (98) has other uses, particularly when used in an iterative MCSCF schene to find an optimum unitary transformation that will yield a "better" set of orbitals based on the CI wavefunction. Also, when the MCSCF wavefunction is converged, the lagrangian will be symmetric (i,e. $x_{i r}=x_{r i}$, He are currently developing and implementing this MCSCF method, and we will present a more detailed description in section IV D.

The advantages of the above theoretical developrents cannot be overestimated. Practical investigations 65,40 emphasize the enormous difficulty of locating transition states, let alone reaction paths, for organtc molecules. The possibility of having accurate forces for CI wavefunctions will mean that advanced numerical techniques ${ }^{66}$ can be applied to these forces and force constants to find reaction paths and transition states with a nininum of computational effort. 


\section{B. Generation of the One- and Two-Particle Density Matrix}

The use of one- and two-particle density matrices allows the energy contribution to be found easily for all ( $3 \mathrm{~N}$ ) different derivatives, since the density matrices are independent of any particular derivative. In addition to determining CI energy derivatives with respect to nuclear position they can also be combined with any other set of integral derivatives to find other CI enengy derivatives (e.g. electric field strength). The one- and two-particle density matrices are given by equations (93) and (94). The coupling coefficlents $b_{I J}^{i j k \ell}$ and $a_{I j}^{i j}$ are exactly those that are used in determining the CI energy, which were defined by loops. The simplest method for generating the density natrices is to generate these sane loops. The onty difference here is that once a loop has been generated it is processed differently. This allows the density matrix to be generated wth the loop-driven algorithm. The effort required to compute the density natrices in this manner is only slightly longer than the effort required to generate the corresponding diagonalization tape.

In the diagonalization tape generation step, loop coefficients are combined with appropriate integrals to form an overail $100 \mathrm{p}$ value. This Ioop value is then used a number of times, determined by the loop breakdown algorithm, for each diagonalization iteration. In generating the density matrices, this process is reversed. When a loop is generated, the loop breakdown algorithn is used first to determine the total loop contribution (d). This total loop contribution is simply a sum of the products of $C_{I}$ and $C_{J}$ for each separate loop contribution and is given by: 
0 i

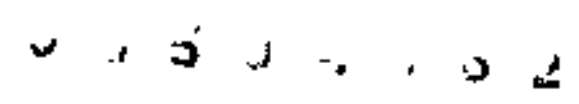

$$
d=\sum_{j}^{\bar{x}_{h}} \sum_{k}^{x_{t}} C_{Y\left(z_{h}+m_{l}+k\right)+j} \cdot C_{Y\left(z_{h}+m^{+} l^{+k}\right)+f}
$$

where $n_{\ell}$ and $m^{\prime}{ }_{\ell}$ are the we1ghts of each branch of the 1oop, $z_{h}$ is the primary upper walk weight, $\bar{x}_{h}$ and $x_{t}$ are the number of upper and lower walks respectively, and $Y$ is the indexing array. Once the total loop contribution has been determined, its product with the loop coeffi. clents is added to correct density matrix elenent terns.

The density matrix elements here play the same role that the mo integrals play in the diagonalization tape generation step. These elements are stored in the same manner and a particuiar density matrix elenent can be found with the integral storage offset arrays by equation (59). Using the same storage method allows a block of density matrix elements to be computed simultaneously with the loopdriven algorithm. 


\section{Transformation and Partial Transformation of the Two-Particle Density Hatrix}

Since the density matrices are generated in the mo basis and the integral derivatives are created in the $\mathrm{AO}$ basis, either the integral derivatives, or the density matrices must be transformed to the other basis. If only one derivative (or degree of freedon) is investigated, then either choice is acceptable. On the other hand, when several sets of integral derivatives are to be computed, it is much more efficient to transform the density matrices to the AO basis as expressed by equations (95) and (96), The nethods required to process these equations are almost identical to the methods for the four-index integral transformation step, with only minor modifications. One such modification is that the initial steps required for this transfornation step, which include reading and sorting the wo one- and two-electron integrals, are identical to the initial steps of the methods required to generate the Lagrangian. Thus the computational mothods for these rather distinct steps can be efficiently conbined together.

Arother important modification involves the storage structure of the firal Ao density matrix elements. Since the integral derivatives are computed using an adapted version of HONDO whith generates integral derivatives with the method of Rys polynomials, ${ }^{67}$ the density matrix elements must be stored by the same blocking structure that the integrals are generated by, This can be done by simply adding an additional sorting step after the transformation. A more efficient method involves producing the transformed AO density matrix elements in the sequence that they are needed. This avoids an extra major sorting step with only a modest increase in the conplexity in the 
U.

transformation algorithm.

It is also possible to eliminate a large portion of this transformation given by equation (96). This advancement which describes an aIternate nethod to conpute the contribution of the all-external two-electron density matrix elements, is analogous to the avoidance of the full integral transformation in section III $M$. By conputing the intermediates $\mathrm{F}_{\mathrm{W}}^{\lambda \nu}, \mathrm{F}_{\mathrm{W}}^{\lambda \nu}, \mathrm{F}_{\mathrm{X}}^{\lambda \nu}$ as given by equations (74-76), the allexternal contribution of the two-particle density matrix over $\mathrm{AO}^{\prime} \mathrm{s}$ can be expressed as

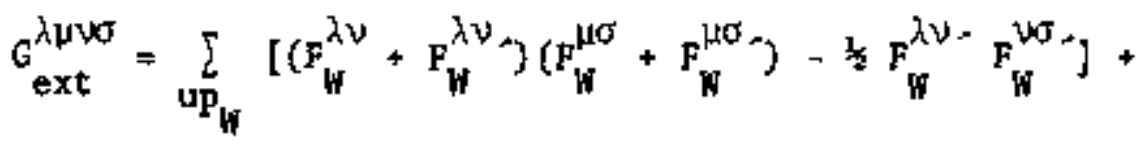

$$
\begin{aligned}
& \sum_{\mathrm{x}} \mathrm{F}_{\mathrm{X}}^{\lambda \nu} \mathrm{F}_{\mathrm{X}}^{\mu \sigma \sigma}
\end{aligned}
$$

This step is of the order $\left(n^{2}{ }^{4}\right)$ and could greatly reduce the effort required to obtatn $\mathrm{E}^{\mathrm{B} \text {, }}$ given by equation (97).

Another potentially very useful approximation is the use of frozen core orbitals and deleted virtual orbitals. The approximation presented here treats the frozen core orbitals as they would act in a gradient procedure at the SCF leveI. In other words the contribution of $\mathrm{E}^{\mathrm{a}}$ given by equation (101) is replaced for frozen core orbitals by

$$
E_{\text {core }}^{\text {as. }}=\sum_{i=\text { frozen core }} \varepsilon_{i} s_{i i}^{2}
$$

where $\varepsilon_{1}$ is the orbital energy of orbital $i$ and $S^{2}$ is the overlap derivative matrix. This is identical to the expression used for all doubly occupied orbitals in an SCF gradient procedure. The contribution of $\mathrm{E}^{\mathrm{a}}$ is ignored for deleted virtual orbitals. The use of 
this approxination implies that any 10 density natrix elenents that represent these orbitals need not be computed provided that these same orbitals were frozen core or deleted virtual orbitals within the CI calculation. This approximation can substantially reduce the (m) process required for both the density satrix transformation and the generation of the Lagrangian. It is important that the $\mathrm{E}^{\mathrm{a} \text {, term }}$ given by equation (97) is not altered by this approximation. Since the NO density matrix elenents that represented frozen core orbitals were never computed, their contribution to the AO density matrix must be included directly. This can be accomplished by expressing the full AO density matrices as

$$
\begin{aligned}
& G_{\lambda \mu \nu \sigma}=G_{\lambda \nu v \sigma}^{1}+2 P_{\lambda \mu} P_{V \sigma}+P_{\lambda \nu} P_{\mu \sigma}-1_{2} P_{\lambda \sigma} P_{\mu \nu}+P_{\lambda \mu} Q_{\nu \sigma}^{\prime}+Q_{\lambda \mu}^{\prime} P_{\nu \sigma} \\
& -1_{4} P_{\lambda \nu} Q_{\mu \sigma}^{\prime}-1_{4} Q_{\lambda \nu}^{\prime} P_{\mu \sigma}-4 P_{\lambda \sigma} Q_{\mu \nu}^{\prime}-\frac{1}{2} Q_{\lambda \sigma}^{\prime} P_{\mu \nu}
\end{aligned}
$$

and

$$
Q_{\lambda \mu}=Q_{\lambda \mu}^{+}+2 P_{\lambda \mu}
$$

where $Q^{\prime}$ and $G^{\prime}$ are the one- and two-particle density matrices in the AO basis constructued only fron those orbitals that are inciuded in the $C I$ and $P_{\lambda \mu}$ is the frozen core density natrix defined as

$$
P_{\lambda \mu}=\sum_{i=\text { frozen core }} c_{\lambda i} c_{\mu j}
$$

The usefulness of this approximation will depend on a number of factors. The main trade-off here is between computation efficiency and accuracy. It is hoped that the error introduced by this approximation will be less than one micro Hart ree for most orbitals that can 
$0 j \cdot 2+j, j, j$

be considered to be part of a frozen core. The capability to apply this approxination already exists within oux CI gradient system. 


\section{Generation and Use of the Lagrangian Matrix}

The initial step in generating the lagrangian given by equation (98), is to process a sort on both the density matrix elenents and the $M O$ integrals. This sort is needed because the density natrix elements $G_{i j a b}$ for all ij for a fixed ab must be present with the wo integrals [kl;ab] for all $\mathrm{k} \ell$. The contribution to the Lagrangian for this fixed $a b$ is then found by a simple watrix multipiy

$$
x_{i r}(a b)=\sum_{j} G_{i j a b}[r j ; a b] .
$$

The methods to process this matrix multiply are slightly nodified because both $G_{i j a b}$ and $[k l ; a b]$ are stored in lower triangular form. The procedure to generate the Lagrangian is conbined with the procedure to transforn the density matrices to eliminate an extra sorting step. The diagonal elements of the Lagrangian are roughly equivalent to the product of the orbital energy and the orbital occupancy of that orbital. The off-diagonal elements correspond to the interaction between two orbitals.

Once the Lagrangian is formed it is used to find the CI energy gradient contribution through equation (101). As mentioned earlier, it is also possible to use the Lagrangian to find the MCSCF wavefunction. $^{68}$ The CI energy can be expressed in a simple form as

$$
\left.E_{C I}=\sum_{i j k \ell} G_{1 j k \ell}[i j ; k \ell]+\sum_{j j} Q_{i j}<\dot{i}|\hat{h}| j\right\rangle
$$

If a unitary transformation was applied to the orbital basis to find a "better" set of orbitals, the CI energy could then be approximated by 
$0 ;, 2, j, j, j, j$

$E \hat{\imath}_{i j k \ell} \sum_{a b c d} a_{i j k \ell} J_{a 1} U_{b j} U_{c k} U_{d \imath}[a b ; c d]+\sum_{i j} \sum_{a b} Q_{i j} U_{a i} U_{b j}\langle a|\hat{h}| b\rangle$

This is only an approxination because the density matrices $G$ and $Q$ depend on the orbital basis in a complex nanner. If the unitary transformation $\mathrm{U}$ was close to unity, then this approximation would be fairly good. This suggests an iterative procedure to find the MCSCP wavefunction. If for any CI calculation the transformation $\underset{\sim}{\mathrm{U}}$ could be found that ninintzes the energy through equation (110), then the $C I$ calculation could be repeated in this new basis (i.e. compute the correct density matrix for this new basis). This pracedure could then be repeated until the transformation ratrix approaches unity, which should also yield the MCSCF wavefunction.

The problen then becones to find a procedure that can deternine the "best" unitary transformation each iteration within this procedure. By assuming that $\mathrm{U}$ is fairly close to unity, $\mathrm{U}$ can be represented as

$$
\underset{\sim}{\mathrm{U}}=\underset{\sim}{\mathbf{v}}+\underset{\sim}{\mathrm{U}}
$$

When this is substituted into equation (110) and all higher terms in $\mathrm{U}^{1}$ are ìnored, then the energy can be further approxinated as

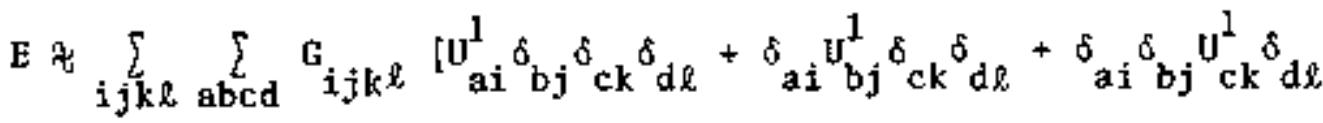

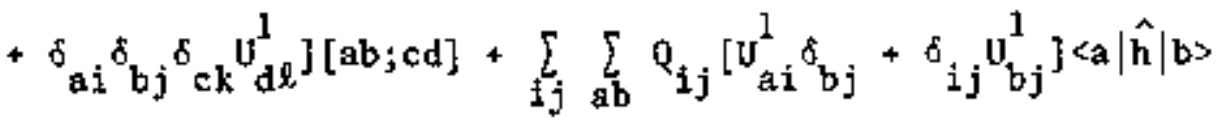

whtch can be simplified to

$$
\left.E \approx \sum_{i j k \ell r} G_{i j k \ell}\left(4 v_{i r}^{1}\right)[r j ; k \ell]+\sum_{i j r} Q_{j j}\left(2 v_{i r}^{1}\right)\langle r|\hat{h}| j\rangle\right) .
$$


Since the Lagrangian is defined as

$$
x_{i r}=\sum_{i j k \ell} 4 G_{i j k \ell}[r j ; k \ell]+\sum_{j} 2 Q_{i j}\langle r|\hat{h}| j\rangle
$$

equation (112) can be further reduced to

$$
E \approx \sum_{i r} x_{i r} U_{i r}^{1}=\sum_{i r} x_{i r}\left(u_{i r}-\delta_{i r}\right)
$$

Misinizing the energy through equation (115) is equivalent to finding the unitary transformation $\mathrm{U}$ that minimizes

$$
E^{\prime}=\sum_{i r}^{L} x_{i r} U_{i r}
$$

This can be done a number of ways. One such approach involves the use of a penalty function which blows up rapldly when $U$ becomes non-unitary. This is done by solving a sinilar problem such as mininizing

$$
E_{p}^{\prime}=\sum_{i r} x_{i r} J_{i r}+P(\underset{\sim}{T})
$$

where $\mathrm{P}$ is the peralty function and $\mathrm{T}$ is given by

$$
T=U_{\sim}^{+} U
$$

One example of a penalty function is

$$
P(T)=a\left(\sum_{i} \exp \left[\left(1-T_{1 i}\right)^{2}\right]+\sum_{i \neq j} \exp \left[\left(T_{i j}\right)^{2}\right]-N^{2}\right)
$$

where $\mathrm{N}$ ts the dimension of $\mathrm{T}$. This penalty function will be zero if $U$ is unitary and be large whenever $U$ deviates sufficiently from a unitary matrix. Equation (117) can now be solved as an unconstrained 


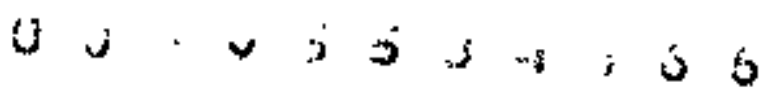

problen. Another approximate method which is much simpler involves the direct diagonalization of a matrix constructed from the $X$ matrix that will give a good unitary transformation.

The computational effort required for each iteration of this MCSCF scheme will be roughty the same as with other MCSCF procedures $[i * \theta$. dominated by the integral transformation step). We have also implemented the capability to treat certain orbitals as part of a frozen core or as deleted virtuals by not allowing these orbitals to "mix" with the renaining orbital space in the MCSCF iterations. This approximation can substantially reduce the $\left(\mathrm{H}^{5}\right)$ integral transformation effort required each iteration.

The MCSCP procedure presented here allows the rigorous optimization of orbitals for any set of configurations that can be used in a CI calculation. These techniques can also be advanced for excited state calculations. We expect that MCSCF calculations involving 10000 configurations will become routine with our system. 


\section{E. Generation and Use of the Integral Derivatives}

The integral dertvatives are generated using an adapted version of the HONDO SCF gradient progran. ${ }^{67}$ The only portions of this program that have been modified are the section that interfaces the progran to the rest of the progran system and the sections of the program that process the conputed integral derivatives. Each integral $\langle\lambda|h| \mu\rangle^{\theta}$ and $[\lambda \mid i w]^{a}$ are processed in two different ways to find the contribution to both $\mathrm{E}^{a}$, and $\mathrm{E}^{\mathrm{a}}$, given by equations (97) and (101). The integral derivatives are first combined with the appropriate density matrix elenent and processed through

$$
E^{2}=\sum_{\lambda \mu} Q_{\lambda \mu}\langle\lambda|h| \mu\rangle^{a}+\sum_{\lambda \mu \nu \sigma} G_{\lambda \mu \nu \sigma}[\lambda \mu ; v \sigma]^{a} .
$$

The integral derivatives are also required to process the coupled. perturbed Hartree-Fock equations. For a closed shell singlet calculation they are processed through

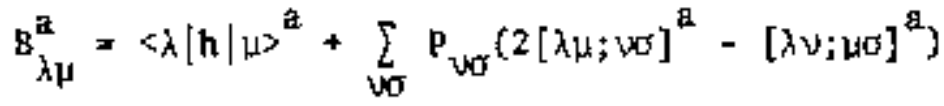

which is simply a Fock matrix constructed from int egral derivatives instead of ordinary integrals. For other types of SCF reference states more than one of these Fock natrices constructed from integral derivatives will be required. In any case, there must be at least one such matrix for each of the ( $3 \mathrm{~N})$ nuclear perturbation under consideration. Since all of these matrices must be maintained within internal storage (core), this applies a modest limit to the size of our calculations. The use of these Fock matrices constructed from integral derivatives will be described in the next section. 


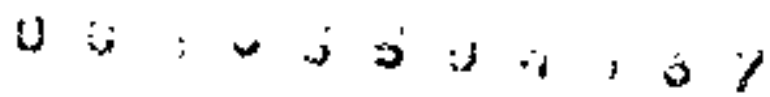

F. Coupled Perturbed Hartree-Fock

The CPHF equations are solved to find the energy derivative contribution of $\mathrm{E}^{\mathrm{a} \alpha}$ given by equation (101). The first order changes in the molecular orbitals $v_{i r}^{a}$ are given by Gerratt and Mil1s ${ }^{62}$ for closed shell RHF as

$$
U_{r s}^{a}=\left(F_{r s}^{a}-\varepsilon_{s} s_{r s}^{a}\right) /\left(\varepsilon_{s}-\varepsilon_{r}\right)
$$

where $\varepsilon_{r}$ is the orbital energy of orbital $r, s^{a}$ is the derivative of the overlap matrix, and $\underset{\sim}{\mathrm{F}}$ is the derivative of the Fock matrix given by

$$
\begin{aligned}
F_{r s}^{a} & =\left\langle r|h| s^{a}+\sum_{k}^{o c c}\left(2[r s ; k k]^{a}-[r k ; s k]^{a}\right)\right. \\
& +\sum_{k}^{o c c} \sum_{b}^{v i r t}(4[r s ; k b]-[r k ; s b]-[r b ; s k]) v_{k b}^{a} \\
& =\sum_{k}^{o c c} \sum_{l}^{o c c} s_{k l}^{a}(2[r s ; k l]-[r k ; s \ell])
\end{aligned}
$$

The difficulty with this representation is that $U$ appears on both sides of this equation. Straightforward methods to solve this equation have been applied by Pople and coworkers. ${ }^{41}$ We find it more useful to rewrite equation (101) in a symnetric and antisynmetric contribution

$$
E^{a}=\sum_{i r} x_{i r}^{+}\left(-s_{i r}^{a} / 2\right)+\sum_{i r} x_{i r}^{-} u_{i r}^{a-}
$$

where

$$
x_{i r}^{+}=\left(x_{1 r}+x_{r 1}\right) / 2,
$$




$$
\begin{aligned}
& x_{i r}=\left(x_{i r}-x_{r i}\right) / 2, \\
& v_{i r}^{a-}=\left(v_{i r}^{a}-v_{r i}^{a}\right) / 2
\end{aligned}
$$

and

$$
v_{i r}^{a+}=\left(u_{i r}^{a}+u_{r i}^{a}\right) / 2=-s_{i r}^{a} / 2
$$

The second part of equation (128) is given since $\mathrm{v}_{i r}^{\mathrm{a}}$ is constrained such that

$$
v_{i r}^{a}+u_{r i}^{a}+s_{i r}^{a}=0
$$

Rewriting equation (122) in this new basis we find that $\mathrm{u}_{\mathrm{rs}}^{\mathrm{a}-}$ is given by

$$
\mathrm{u}_{r s}^{\mathrm{a}-}=\left[z_{r s}^{\mathrm{a}}-\left(\varepsilon_{s}+\varepsilon_{r}\right)\left(s_{r s}^{\mathrm{a}} / 2\right)\right] /\left(\varepsilon_{s}-\varepsilon_{r}\right)
$$

For this representation $\mathrm{F}_{\mathrm{rs}}^{\mathrm{a}}$ is given by equation (123), but in the new representation it is better expressed in a form dependent on $\mathrm{u}^{\mathrm{a}-}$ as

$$
\begin{aligned}
\mathbf{F}_{r s}^{\mathrm{a}} & =\mathrm{B}_{\mathrm{rs}}^{\mathrm{a}}+\sum_{\mathrm{k}}^{\mathrm{occ}} \sum_{\mathrm{b}}^{\operatorname{virt}}(4[\mathrm{rs} ; \mathrm{kb}]-[\mathrm{rk} ; \mathrm{sb}]-[\mathrm{rb} ; s \mathrm{k}]) \mathrm{u}_{\mathrm{kb}}^{\mathrm{a}-} \\
& =\sum_{\mathrm{k}}^{\circ \mathrm{cc}} \sum_{\mathrm{t}}^{\mathrm{all}}(4[\mathrm{rs} ; \mathrm{kt}]-[\mathrm{rk} ; \mathrm{st}]-[\mathrm{rt} ; s \mathrm{k}])\left(s_{\mathrm{tk}}^{\mathrm{a}} / 2\right)
\end{aligned}
$$

where $B_{r S}^{a}$ is obtained by the transformation

$$
\mathbf{B}_{r s}^{\mathbf{a}}=\sum_{\lambda \mu} \mathbf{c}_{\lambda \mathbf{r}} \mathrm{c}_{\mu s} \mathbf{B}_{\lambda \mu}^{a}
$$

and $B_{\lambda \mu}^{a}$ is given by equation (121). The $\mathrm{V}^{\mathrm{a}+}$ contribution has been included in the last term. 
U.

As demonstrated by Pople, ${ }^{41} \mathrm{y}^{\mathrm{a}-}$ can be found by solving the coupled equations

$$
(I-A) v^{a-}=U_{-0}^{a}
$$

where

$$
A_{(a i, b j)}=(4[a i ; b j]-[a j ; b i]-[a b ; i j]) /\left(\varepsilon_{i}-\varepsilon_{a}\right)
$$

and

$$
\begin{aligned}
& U_{D_{(a 1)}^{a}}^{a}=\left[c_{a}|h| i\right\rangle^{a}-\sum_{k}^{o c c}\left(2[a 1 ; k k]^{a}-[a k ; i k]^{a}\right) \\
& -\frac{\left(\varepsilon_{i}-\varepsilon_{q}\right)}{2} s_{a i}^{a}-\sum_{k}^{a c c} \sum_{t}^{a l l} s_{t k}^{a}\left(\frac{t a}{2}\right)(4[a i ; t k]- \\
& -[a k-t i]-[a t ; i k])] /\left(\varepsilon_{1}-\varepsilon_{a}\right) \text {. }
\end{aligned}
$$

The matrix $\underset{z}{A}$ is a non-symetrical square matrix that has the dimensions of the product of the number of occupied orbitals (excluding frozen core orbitals used with the frozen core approximation) and the number of virtual orbitals (again excluding the deleted virtuals). $\underline{u}^{\text {an }}$ and $\mathrm{U}^{2-}$ are column vectors of the sane dimension. This equation must be solved for each different derivative under consideration.

Once equation (133) has been solved the complete v $^{\text {a- }}$ matrix can be found by equations (130) and (131), except that we are not really interested in computing the $\mathrm{U}^{\mathrm{a} *}$ natrix, but only its contribution to the energy gradient given by equation (124). The total contribution to the energy gradient is found in a straightforward manner for all terms except the contribution derived from the second ters of equation (131). This is the only tern which requires the solution of the CPHF equations and its contribution can be expressed as 


$$
\begin{aligned}
z^{a}-s & =2 \sum_{r>s}\left[x_{r s}^{-} /\left(\varepsilon_{s}-\varepsilon_{r}\right]\left[\sum_{k}^{o c c} \sum_{b}^{\operatorname{virt}} u_{b k}^{a-}(4[r s ; b k]\right.\right. \\
& -[r k ; b s]-[r b ; s k])]
\end{aligned}
$$

Which is treated separately, thus avoiding the explictt calculation of $\mathrm{U}^{\mathrm{a}-}$ for the parts that do not connect the occupied and virtual spaces.

Pople ${ }^{41}$ presented an innovative iterative method to solve the coupled equations given by equation (133). His method has many similarities to the Dayidson diagonalization nethod, ${ }^{56}$ except that the method solves a set of simultaneous equations. (In fact, to implement this method we simply made minor modifications to our diagonalization routine.) For each nuclear variable he defines an increasing set of orthogonal vectors given by

$$
\underset{\sim 0}{\mathbf{a}}={\underset{\sim}{\mathrm{d}}}_{0}^{\mathrm{a}-}
$$

and

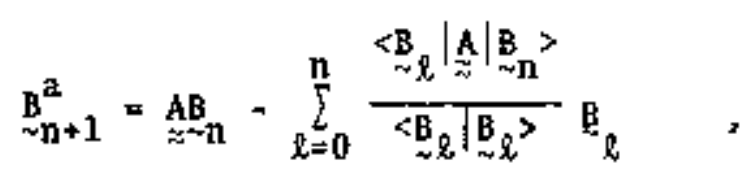

where $n$ increases by one each iteration. The solution is approximated by solving a much smaller set of simultaneous equations given by

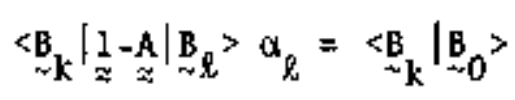

for 2 and $k=0, n$ and then

$$
\operatorname{u}_{n=1}^{a-} \sum_{x=0}^{n} a_{\ell} \quad B
$$

The time-consuring step for this procedure is the effort required to 


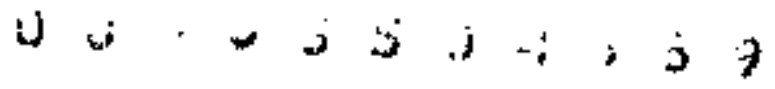

multiply the matrix $\underset{\approx}{A}$ by the vector $\underset{\sim}{B}$ each iteration.

This concept can be improved even further by considering that the different solutions to equation (133) for each different derivative will not be orthogonal. Thus, by approximating each solution with the same set of orthogonal vectors it is possible to reduce the computation effort for this step. The savings can then be found in that the number of matrix multiplies per iteration can be stbstantially reduced (typically half of the number of nuclear variables) with only a modest increase in the number of iterations. This is done by defining an orthonormal set of $n$ vectors $\underset{\sim i}{b}$ and vector products $a_{i}$ given by

$$
\underset{\sim i}{\sigma}=\underset{z}{(1-A)})_{\sim i}
$$

For each nuclear variable, solve a small set of coupled equations

$$
\left\langle\underset{\sim k}{b} \mid \sigma_{\ell}\right\rangle \alpha_{\ell}^{a}=\underset{\sim k}{b}\left|\underset{\sim}{v^{a-}}\right\rangle
$$

for $k$ and $l=1, n$. The approximate solution to equation (133) is given by

$$
\mathrm{u}^{\mathrm{a}-} \sum_{\mathrm{k}=1}^{\mathrm{n}} \mathrm{a}_{\mathrm{l}}^{\mathrm{a}} \mathrm{b}_{\mathrm{d}}
$$

and the correction vector to improve convergence is given by

$$
\underset{\sim n}{b^{a}}=b_{\sim n}^{a} \cdots-\sum_{\ell=1}^{n}<b_{d} \mid b_{n}^{a}>>b_{k}
$$

where

$$
b_{n}^{a}=\sum_{l=0} \alpha_{l}^{a} \sigma_{l}
$$

This will yield a set of (3N) new correction vectors which are neither normatized nor orthogonal to each other. From this set, approximately 
(3N/2) orthonormal vectors are produced and added to the set of $\pi$ vectors. These vectors, which are linear combinations of the $b_{n}^{a}$, vectors, are chosen so as to nost improve the nuclear degrees of freedon which had poorer convergence (i.e, where $\left.\vec{b}_{n}^{a}-b_{n}^{a}\right\rangle$ is large), thus after several such iterations all (3N) solutions will be equally converged. The initial orthonormal set of $b_{i}$ vectors is obtained by orthogonalizing and normalizing the $\mathrm{v}_{0}^{\mathrm{a}-}$ vectors. 


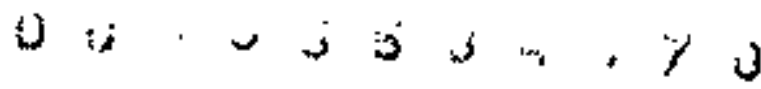

v. GRAPHICAL INITARY GROUP APPROACH CI AND CI ENERGY GRADIENTS

AS A SYSTEM OF PROGRAMS

The methods and algorithns described in the earller sections have been conbined into an effective system of prograns. The system is referred to as the Berkeley Unitary Group Approach Configuration Interaction (BUGACI) system of programs. Figure 6 shows a siaplified schematic of the information flow within this system.

The input to this systere (other than direct user input) is a set of symmetry orbital (SO) integrals, and a flle that contains the SCF transformation natrix and other orbttal information. The progran DRTGEN generates all of the distinct row table arrays as well as the integral sorting arrays. Thts is the only program in the systen that requires any appreciable user supplied input (cards) beyond simple option paraneters. The DRT file is used by many programs in the system.

The program TRANS performs the integral transformation. The algorithm used in this transformation progran differs sonewhat from conventional appraaches in that extra sorting steps have been eliminated, integrals are always stored with lmplicit addressing, and the effective working space is doubled using a scratch file as virtual menory. The "frozen core" contribution is included through the use of a frozen core Fock thatrix as a one-electron effective Hamiltonian. One current drawback to this program is that symmetry is not included explicitly.

The program NENCORE is used to increase the number of frozen core orbitals by including the new frozen orbitals into the effective Hamittonian. Since this process results in a loss of information, this program can only be used to increase the number of frozen core 
orbitals.

The program BUGME generates the diagonalization tape with the loop-driven algorithm. This progran has the capability of generating three sequential tapes; one for diagonal loops, one for off-diagonal loops, and the last one is used to store only "important" off-diagonal loops. The diagonalization program (DIAG) can iterate rapidly reading only the diagonal elements and the inportant off-diagonal loops and when convergence is near, the progran can switch to the full offdiagonal Joops tape. The program DIRECT avoids the storage of the diagonalization tape, and constructs all of the loops each diagonalization 1teration.

After the CI has been completed, the progran DENSITY can obtain the one-particle density matrix which can be used to obtain CI properties (CIPROPS), or used to obtain a set of natural orbitals. This set of natural orbitals can be used to transform the 10 integral list to a new basis (the original integral tape is always used to elirinate excessive error accumulation, with the program NOTRANS. This progran is a1most fdentical to TRANS and is used for natural orbital iterations in this way.

The program TWOPDN generates the one- and two-particle density matrices fron the CI vector. These can be used to find an optimum orbital transformation matrix with the program SUPERCI. By transforming the integrals to this optimum basis and iterating, the MCSCF wavefunction can be found.

The one- and two-particle density batrices can also be used to obtain CI energy gradients with respect to all nuclear perturbations. This is done by transforming the MO density matrices to the AO basis 
$0, i, j ; 1,1$

with the program TRANSDU, and combining then with the integral derivatives generated by the program CIDER. The progran CIDER also conputes the integral derivative fock matrices required to solve the CPHF equations which are processed in the progran CICPHF. The progran CICPHF combines the separate energy derivative contributions and produces the final result. The gradient system in its present form is restricted to closed shell RHF wayefunctions, or converged MCSCF wavefunctions.

This systen of programs has not beer completed at the time of thas writing, and will tndoubtedly undergo substantial modifications, inproverents, and additions in the future. It is expected (and hoped) that this system of programs will be maintained and used for a number of years. 
VI. APPLICATIONS 


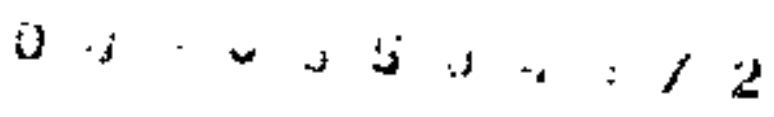

\section{A. Exarples and Tining Results}

One of the more inportant aspects to this work is the reporting of a series of examples including timing breakdowns and comparisons between the current version and the preliminary version of this method, as well as with other CI methods. Also reported, in addition to timing results, are some statistical data on generated loops. All timing resuls are given in minutes on the Harris 6024/4 (about a factor of 27 slower than the CDC 7600 ).

Since our prelininary implementations, we have more than doubled the efficiency of the prograns (excluding the diagonalization timings and integral transformation). There are a great number of factors that contribute to this increase in efficiency. The more important of these are: 1) the use of the new segment coefficients, 2) reduction in searching for acceptable segments due to the use of new coefficients and an additional tost to determine if enough electrons remain in lower orbitals, 3) elimination of the buffer packing and unpacking required in the formula tape version, 4) elimination of coding loop coefficients and the assoctated packing operations, 5) use of the implicit treatment of the all-external loops, and 6) an sssortment of other minor inprover ments in many other areas.

The first example involves the closed shell ground state of $\mathrm{BH}_{3}$ in $\mathrm{C}_{2 v}$ symmetry. It is a small calculation and reported here primarily for the comparison of CI methods as well as detailed timing breakdown of all aspects of the dlagonalization tape generation depicted in Figure 5. Although this is a rather small calculation, it is not expected that the relative proportions of the different sections will 
change substantially as one goes to larger calculations. The second example involves the ${ }^{1} \mathrm{~A}_{1}$ state of $\mathrm{CH}_{2}$ with a fairly extensive basis set. This example gives a direct comparison of the integral driven CI nethod ${ }^{9}$ and the GUGA method for a noderately 1 arge calculation. The thitd exanple is a calculation of the ${ }^{1_{B}}$ (V state) of ethylene. Example three consists of the Hartree-Fock interacting configuration space from three open-shell singlet reference configurations with a double zeta plus polarization diffuse functions basis set.

The fourth example represents a rather large calculation on the $2^{2} A^{\prime}$ state of $\mathrm{SO}_{2}$ at an unsymetrical geonetry $\left[\mathrm{C}_{s}\right.$ symnetry $]$. For this calculation the interacting space from three reference configurations was included after 7 care orbitals and the 7 highest virtagls were deleted. For the basis set used (double zeta plus polarization functions on the sulfur atom] 23,613 spin-adapted Gelfand states were required. Since only $20 K$ real words are available to the user on the Harris miniconputer, the large diagonalizat ion techniques described in section III $L$. were required.

These examples represent only a small subset of the types of calculations possible with the GJGA method as currently implemented.

The timing comparisons given in Examples 1-3 indicate definite trends. One obvious trend is that as the problem size gets 1 arger the GUGA becones increasingly superior to the other methods. This increase in efficiency can be traced to an increase in the size of the secular equation (as opposed to an increase in the number of molecular orbitals). This increase in efficiency can also be understood very easily if the loop statistics are compared, As the number 
of configurations increase, the average number of total contributions for each loop increases. Each of these contributions must be dealt with separately for the conventional $C I$ method and the integral driven CI method. For the GUGA method the time required depends heavily on the nubber of loops generated, not the number of contributions per loop. For this same reason, calculations with little or no spatial symmetry will enjoy an even greater efficiency. Another trend that should be mentioned is that the GIGA nethod becones more efficient compared with the other CI methods, for a given number of configurations, as the number of electrons included in the CI increases.

The loop statistics given in Table $V$ for each case separate the diagonal loops, those loops that contribute to diagonal matrix elements, from the off-diagonal loops. Since the diagonal loops are on1y processed once for a given calculation and the off-dlagonal loops are processed each iteration, it is the off-diagonal loops that determine the computational effort required for the diagonalization. For the lower walks, two values are reported; the average number of lower walks and the percentage of loops with only one lower walk. This is done for upper walks, as woll as for the total number of loop contributions. The large percentage of loops with total contributions of unity suggests that an alternate method of storing and processing these loops is worth considering. 


\section{B. Vertical Electronic Spectrum of Ketene}

Three years ago Dykstra and Schaefer reported a careful SCF study of the low-lying electronic states of ketene ${ }^{69}\left(\mathrm{C}_{2} \mathrm{H}_{2} \mathrm{O}\right)$. However, ro consideration was given to the effects of electron correlation. Using the present GUGA method, it is possible to reliably evaluate correlation effects in little more than the time required for the original SCF studies.

The standard Huzinaga-Dunning contracted Gaussian double zeta basis set was adopted, ${ }^{70}$ as in the previous work of Dykstra and Schaefer and that of Harding and Goddard. ${ }^{71}$ With the three 1s-2ike orbitals frozen (doubly occupled in all configurations), CI was carried out including all single and double excltations. Only the vertical (ground state equilibrium) geonetry was consldered, and

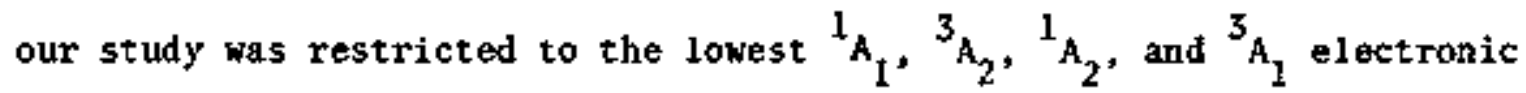
states.

The numbers of spin and space adapted configurations included were 5089, 6300, 6234, and 5981, respectively. For the largest calculation, the ${ }^{3} \mathrm{~A}_{2}$ state, integral computation required 19 minutes, 20 SCF iterations required 29 minutes, the integral transformation 9 minutes, and the unitary $\mathrm{CI}$ a total of 54 minutes. Thus we see that the tine for the CI step is comparable to that for the telatively routine SCF calculation. Since the formula tape required 23 mintues, further CI calculations on the ${ }^{3} A_{2}$ potential energy surface would require on Iy 40 minutes each, including the four-index transformation of two-electron integrals.

The SCF and CI total energies obtained here are -151.67244 and 
$0 ;, 3,1,1,4$

$-151.94659\left({ }^{\mathrm{l}_{\mathrm{A}}}\right),-151.56305$ and $-151.81489\left({ }^{3} \mathrm{~A}_{2}\right),-151.55581$ and $-151.81331\left({ }^{1} A_{2}\right),-151.49968$ and -151.74846 Hartree $\left({ }^{3} A_{1}\right)$. The best previous variational calculations on ketene were those of Harding and Goddard, who report a CI ground state energy of -151.8271 Hartree, or about 0.12 Hartree above the present unitary CI result.

Relative energies are presented in Table VI and compared with those of Dykstra and Schaefer, Marding and Goddard, and the experimental electron inpact results of Prueholz, Flicker, and Kuppermann. ${ }^{72}$ our results provide very strong support for the theoretical predictions of Harding and Goddard, ${ }^{71}$ the largest difference being $0.06 \mathrm{eV}$. In addition the agreenent with the electron impact experiments is as good $( \pm 0.1 \mathrm{eV})$ as could reasonably be expected at this level of theory. 


\section{CONCLUDING REMARKS}

This work has demonstrated that the loop-driven graphical unitary group approach is superior to state-of-the-art conventional configuration interaction (CI) techniques for the description of electron correlation in molecules. Further developments described herein yield an additional factor of two improvement in the effictency of this new method when compared with the preliminary implenentation. Posstbly even nore important is the fornulation of a nethod for a aralytical evaluation of the gradlent of the potential energy. The latter method allows the evaluation of all first derivatives of the potential energy with an amount of effort not more than 2-3 tines that required to obtain a single CI energy on the potential surface.

Some of the material contained in this work has been presented previous $1 y .73,74$ 


\section{REFERENCES}

1. G. Das and A.C. Wahl, Phys, Rev. Lett, 24, 440 (1970).

2. C.F. Bender, S.V. O'Neil, P.K. Pearson, and H.P. Schaefer, Science $176,1412(1972)$.

3. G.C. Lie, J, Hinze, and B. Liu, J. Chem. Phys, $\underline{59}$, 1872 (1973).

4. I. Shavitt, "The Hethod of Configuration Interaction," Modern Theoretical Chemistry, Vol, 3, edited by H.F. Schaefer (Plenum, New York, 1977), pp. 189-275.

5. W. Meyer, Int. J, Quantim Chem, \$s, 341 (1971).

6. R. Ahlrichs, H. Lischka, V. Staemmler, and W. Kutzelnigg, J. Chein. Phys, 62, 1225 (1975).

7. B. Raas, Chen. Phys, Lett, 15, 153 (1972).

8. R.P. Hausman, S.D. Bloon, and C.F. Bender, Chem. Phys, Lett. 32 , $483(1975)$.

9. R.R. Lucchese and H.F. Schaefer, J. Chen. Phys, 68, 769 (1978).

10. C.E. Dykstra, H.F. Schaefer, and W. Meyer, J. Chem. Phys. 65, 2740 (1976).

11. P.S. Bagus, B. Liu, A.D. McLean, and M. Yoshinine, Nave Mechanics: The First Fifty Years, edited by W.C. Price, S.S. Chissick and T. Ravensdale (Butterworth, London, 1973), pp. 99-118.

12. R.J. Buenker and S.D. Peyerimhoff, Theor. Chim. Acta 35, 33 (1974).

13. M.F. Guest and W.R. Rodwell, Mo1. Phys. 32, 1075 (1976); J. Kendrick and I.H. Hillier, Mo1. Phys. 33, 635 (1977).

14. L.E. Meturchie and E.R. Davidson, J. Chem. Phys. 65, 2959 (1977).

15. L.B. Harding and W.A. Goddard, J. Chen. Phys. 67, $1777(1977)$.

16. P.J. Hay and T.H. Dunning, J. Chem. Phys. 67, 2290 (1977). 
17. C.W. Bauschlicher and I. Shavitt, J. Am. Chem. Soc. 100, 739 (1978).

18. R. Seeger, R. Krishnan, and J.A. Pople, J. Chem. Phys. 68, 2519 $(1978)$.

19. B.R. Brooks and H.F. Schaefer, Int. J. Quantum Chem. 14, 603 (1978).

20. C.F. Bender and H.F. Schaefer, J. Am. Chen. Soc. 92, 4984 (1970).

21. M. Moshinsky, in Group Theory and the Many-Body Problen (Gordon and Breach, New York, 1968).

22. I.M. Gelfand and M.L. Zetlin, Dok1. Akad. Nauk SSSR 71, 825, 1017 (1950).

23. J. Paldus, J. Chem, Phys. 61, 5321 (1974); Int. J. Quantum Chem. Symp. 9, 165 (1975); Phys. Rev. A 14, 1620 (1976).

24. (a) I. Shavitt, Int. 3. Quantun Ches. Symp. 11, 131 (1977);

(b) 12,5 (1978).

25. I, Shavitt, "A Short Course on Matrix Elerent Evaluation Using the Unitary Group," Theoretical Atomic and Kolecular Physics Group, Lawrence Livermore Laboratory, May 30 - June 2, 1978.

26. M. Kotani, A. Ameniya, E. Ishiguro, and T. Kimura, Tables of Molecular Integrals (Maruzen, Tokyo, 1955).

27. P.o. Löwdin, Rev. kod. Phys. 36, 966 (1964).

28. H.F. Schaefer and F.E. Harris, J. Comput. Phys. 3, 217 (1968).

29. A. Bunge, J. Chem. Phys, 53, $20(1970)$.

30. R.R. Lucchese, B.R. Brooks, J.H. Meadows, W.C. Swope, and H.F. Schaefer, J, Comput. Phys, 26, 243 (1978),

31. See, for example, S.F. Boys, Proc. Roy. Soc. (London) A217, $325(1953)$. 
32. P. Pulay, pages 152-185 of Volune 4, Modern Theoretical Chemistry, edited by H.F. Schaefer (Plenum, New York, 1977).

33. P. Pulay, Mo1. Phys. 17, 197 (1969); 18, 473 (1970).

34. D. Poppinger, Chem. Phys. Lett. 34, 332 (1975); 35, 550 (1975).

35. H,B. Schlegel, S. Wolfe, and F. Bermardi, J. Chen. Phys, 63, $3632\{1975\}$.

36. A. Kormornicki, K. Ishida, K. Morokma, R. Ditchfield, and $M$. Conrad, Chem, Phys, Lett. 45, 595 (1977).

37. M. Dupuis and H.F. Kıng, J. Chen. Phys. 68, 3998 (1978).

38. Pople, J.A. Seeger, R., and Krishnan, R., Intern. J, Quantum Cher. 11, $149(1977)$.

39. J.S, Binkley, J.A. Pople, and W.J. Hehre, to be published.

40. J.D. Goddard, N.C. Handy, and H.F. Schaefer, to be published.

41. J.A. Pople, invited lecture at March 1979 Sanibel Sympasium, Paln Coast, Florida.

42. J. Gerratt and I. Mills, J, Chem. Phys, 49, 1719 (1968).

43. J.-F. Gouyet, R. Schrammer, and T.H. Seligman, J. Phys. A $\underline{8}, 285$ $\{1975)$.

44. G.W.F. Drake and W, Schlesinger, Phys. Rev. A 15, 1990 (1977).

45. ป. Paldus, Physica Scripta 00, 0000 (1979).

46. I. Shavitt, Annual Report on "New Methods in Computational Quantum Chenistry and their Application on Modern Super-Computers" to NASA Ames Research Center, Jume 29, 1979.

47. C.F. Bender and H.F. Schaefer, J. Chern. Phys. 55, 4798 (1971);

R.R. Lucchese and H.F. Schaefer, J. Amer. Chem. Soc. 99, 6766 (1977). 
48. B.0. Roos and P.E.M. Siegbahn, Int. J. Quantun Chen. (1978).

49, I. Shavitt, Chem. Phys. Lett. 00, 0000 (1979).

50. J. Paldus, Theoretical Chemistry: Advances and Perspectives 2 , $131(1976)$.

51. D.E. Knuth, "Sorting and Searching," The Axt of Computer Programiing, Vol. 3 (Addison-Wesley, Reading, MA, 1973).

52. M. Yoshimine, J. Comput, Phys. 11, 449 (1973).

53. P. Siegbahn, J. Chem. Phys, 70, 0000 (1979) .

54. I. Shavitt, private comunication.

55. P.K. Pearson, R.R. Lucchese, W.H. Miller, and H.F. Schaefex, pages 17I-190 of Minicouputers and large Scale Conputations, Symposium Series 57, editor P. Lykos (American Chemical Society, Hashington, D.C., 1977).

56. E.R. Davidson, J. Conputational Phys. 17, 87 (1975).

57. See for example, G.H.F. Diercksen, W.P. Kraemer, and B.O. Roos, Theoret, Chim. Acta 36, 249 (1975).

58. J. Paldus, in Theoretical Chemistry: Advances and Perspectives, Vo1. 2, edited by H. Eyring and D.J. Henderson (Academic, New York, 1976), p. 131 .

59. W. Meyer, J. Chem. Phys. 64, 2901, (1976).

60. C.E. Dykstra, H.F. Schaefer, and W. Meyer, J, Chem. Phys. 65 , $2740(1976)$.

61. At the SCF level of theory, such gradient procedures are well established. See P. Pulay, pages 152-185 of Volune 4, Modern Theoretical Chemistry, editor H.F. Schaefer (Płenum, New York, 1977). 
62. J. Gerratt and I, Nills, J, Chen. Phys. 49,1719 (1968).

63. B, Roos, Chen. Phys. Lett. 15, 153 (1972).

64. B.T. Sutcliffe, Hethods of Nolecular Quantum Mechanics (Academic Press, London, 1969) .

65. J.A. Pople, R. Krishnan, H.B. Schlegel, and J.S. Binkley, Intern. J. Quantum Chen. 14, 545 (1978) .

65. M.C. Flanigan, A, Komornicki, and J,W. McIver, pages $1-47$ of Volume 8, Modern Theoretical Chemistry, editor G.A. Segal (Plenum, New York, 1977).

67. M, Dupuis and H.F. King, J. Chem. Phys. 68, 3998 (1978).

68. J. Hznze, J. Chen. Phys, 59, 6424 (1973).

69. C.E. Dykstra and H.F. Schaefer, J. An. Chen. Soc. 98, 2689 (1976).

70. S, Huzinaga, J. Chem. Phys, 42, 1293 (1965); T.H, Dunning, J. Chen. Phys. 53, 2823 (1979).

71 , L.B. Harding and W.A. Goddard, J. An. Chen. Soc, 98, 6093 (1976).

72. R.P. Frueholz, W.M. F1icker, and A. Kuppermann, Chem. Phys, Lett. $38,57(1976)$.

73. B.R. Brooks and H.F. Schaefer, J. Chen. Phys. 70, $\$ 092$ (1979).

74. B.R. Brooks, H.D. Laidig, P. Saxe, N.C. Handy, and H.F. Schaefer, To be published In the Proceedings of Nobel Symposium 46 (1979). 


\section{FIGURE CAPTIONS}

Figure 1. A sample Shavitt graph that describes the full CI configuration space for a systea with 3 electrons and 4 orbitals.

Figure 2. A sample one electron 1oop that deptets the contribution of the integra1 $(i|\hat{h}| j)$ to the matrix element $\left\langle w^{\prime}|H| n\right\rangle$.

Figure 3. A simplified schematic of all 14 loop types (and subtypes). Symbols $R, L$, and $W$ refer to raising, lowering, and weight generators respectively. The solid lines refer to the $n$ branch of the loop and the dashed lines refer to the $n$ ' branch. Intermediate lines are partially indicated to show their connections by dots where significant, oven though they are not used in the new coefficient representation. Loop types 9, 10a, and 13a are restricted to avoid contributions to $\left\langle\mathrm{m}^{\prime}|\mathrm{H}| m\right\rangle$ where the lexical order of $m^{\prime}>m$.

Figure 4. Pour sample loops that share a cotmon loop head and depict the effectiveness of the loop-driven algorithm. Once any loop has been generated (solid arcs), only the differing portions of additional loops (dashed arcs) need to be processed.

Figure 5. A detailed timing breakdown in minutes of the major sections of the current verion of the GUGA method for Example I. Also included is the time required to perform the step $\sigma=$ Fic each iteration of the diagonalization. 


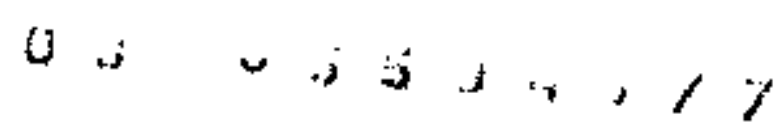

Figure 1.

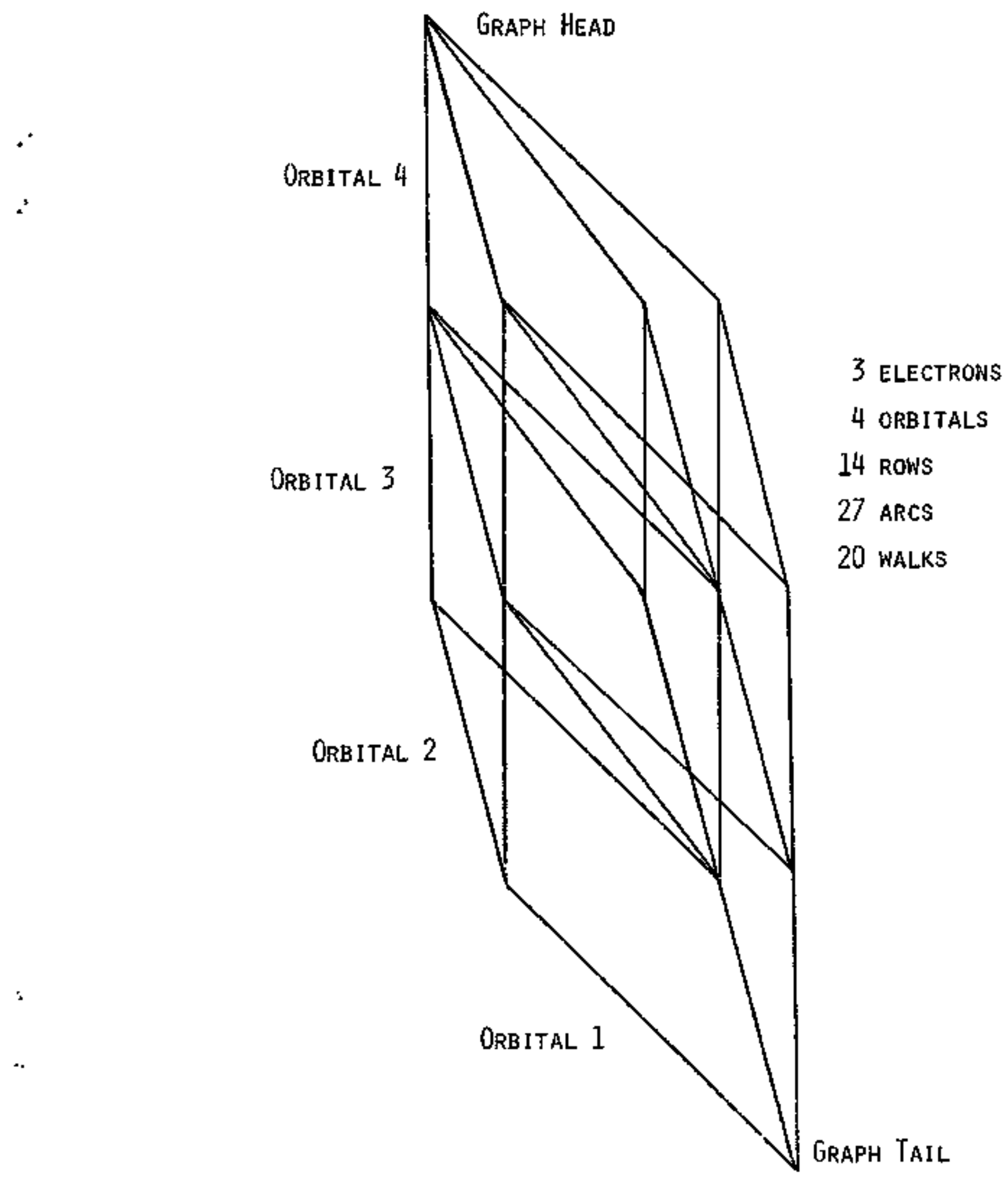


Figure 2.

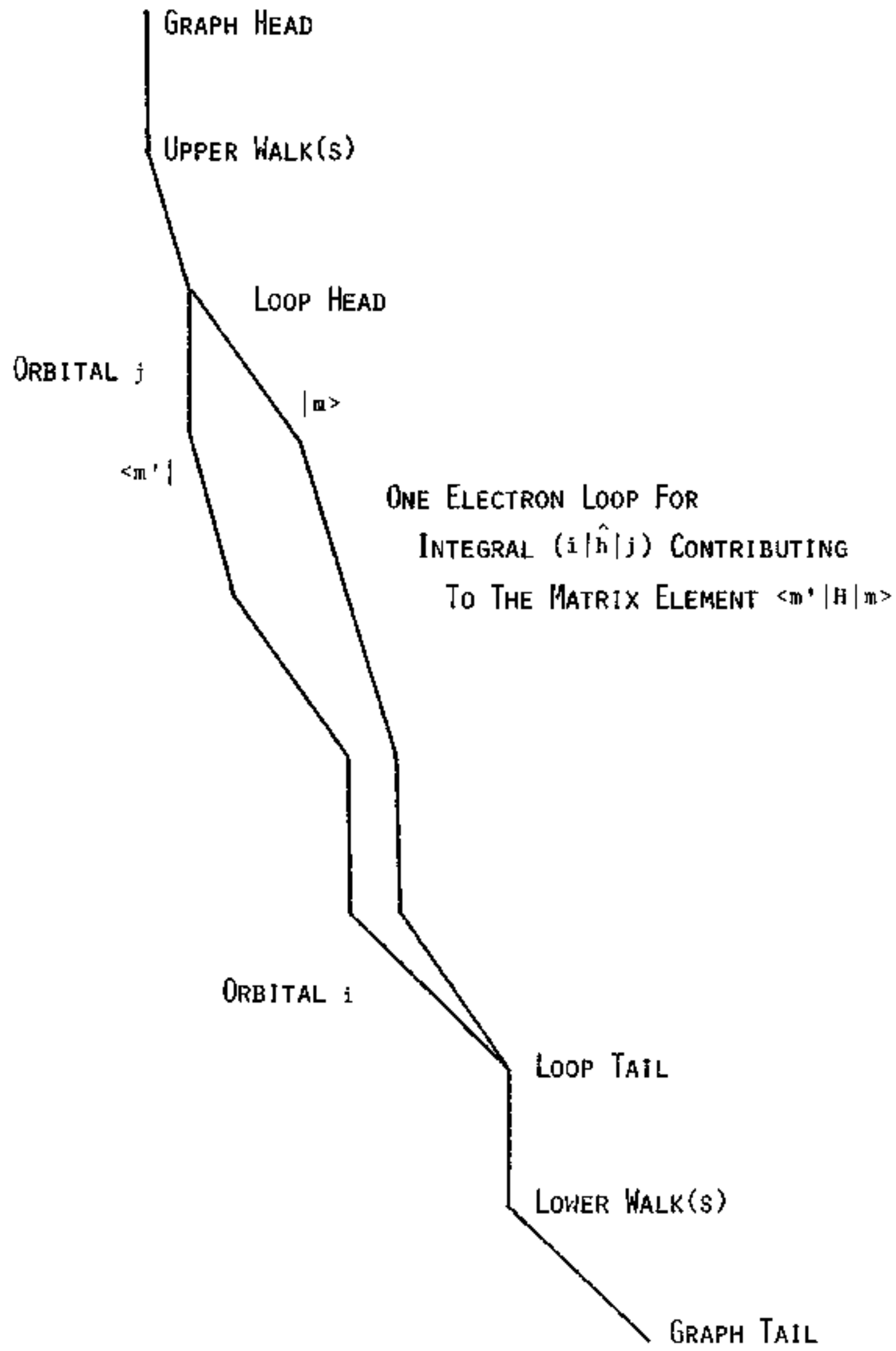


i i $4: 59-7,73$

Figure 3.

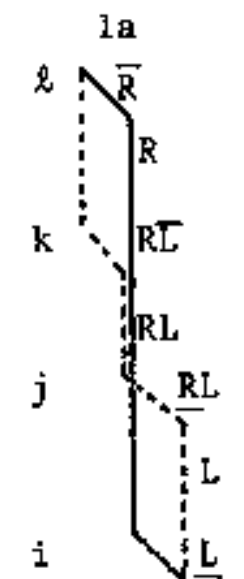

$E_{k i, j \ell} \quad \varepsilon_{j i, k \ell}$ [ik;ji]

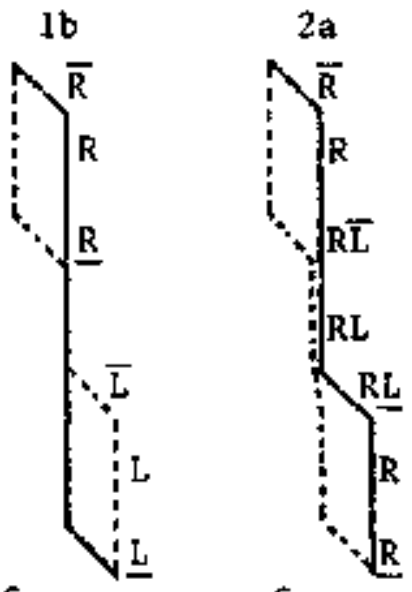

$\varepsilon_{k j, i \ell}$

[il;jk]

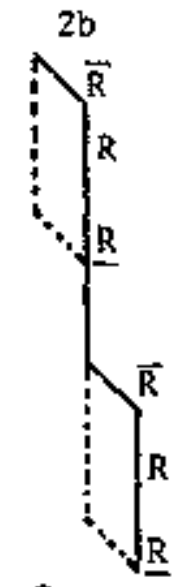

$E_{i j, k \ell}$

[ij;kl]

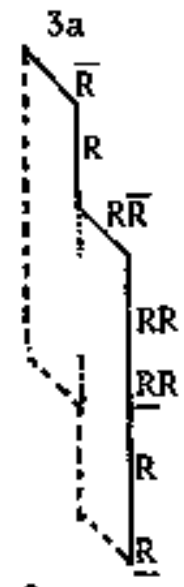

$\epsilon_{i k, j \ell}$

[ik;jl]

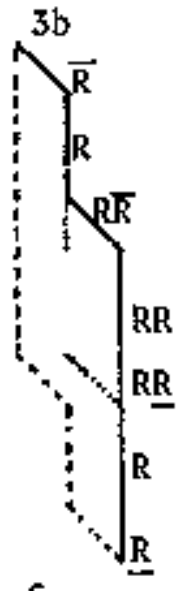

$\varepsilon_{j k, i l}$ [il;jk]
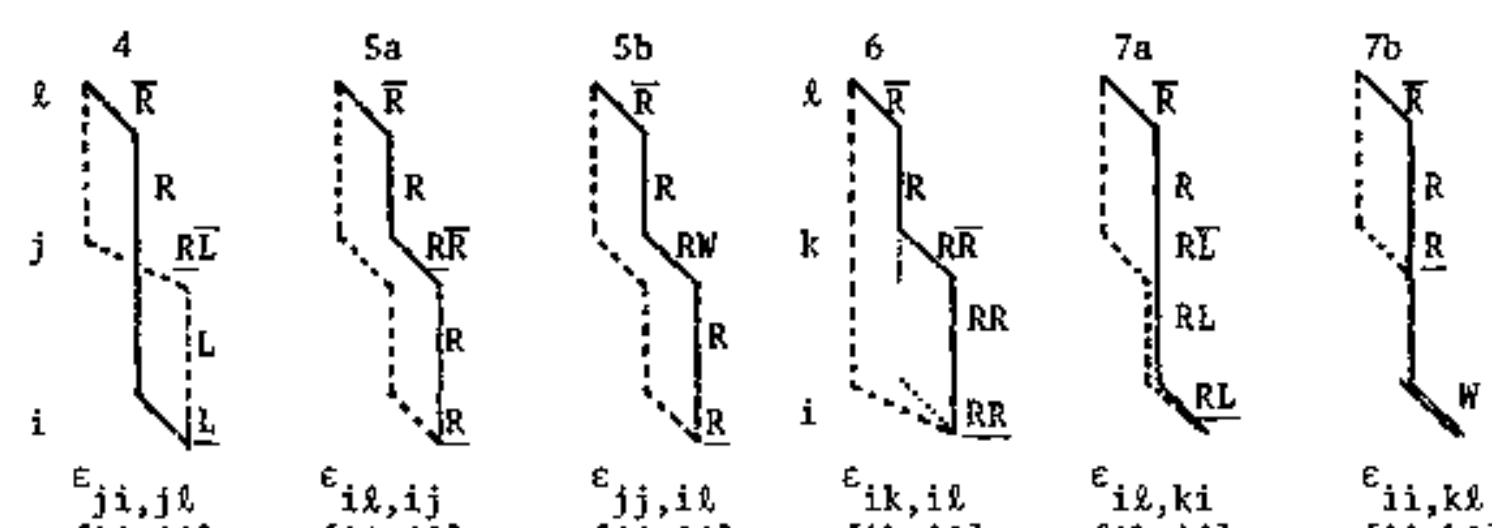

[ij;jl]
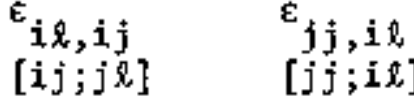

$E_{i k, i \ell}$

$[j j ; i l]$

[ik;il]

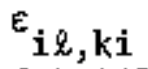

[ik;ilu]

$\varepsilon_{i \mathbf{i}, \mathrm{k} \ell}$

[iz;kl]

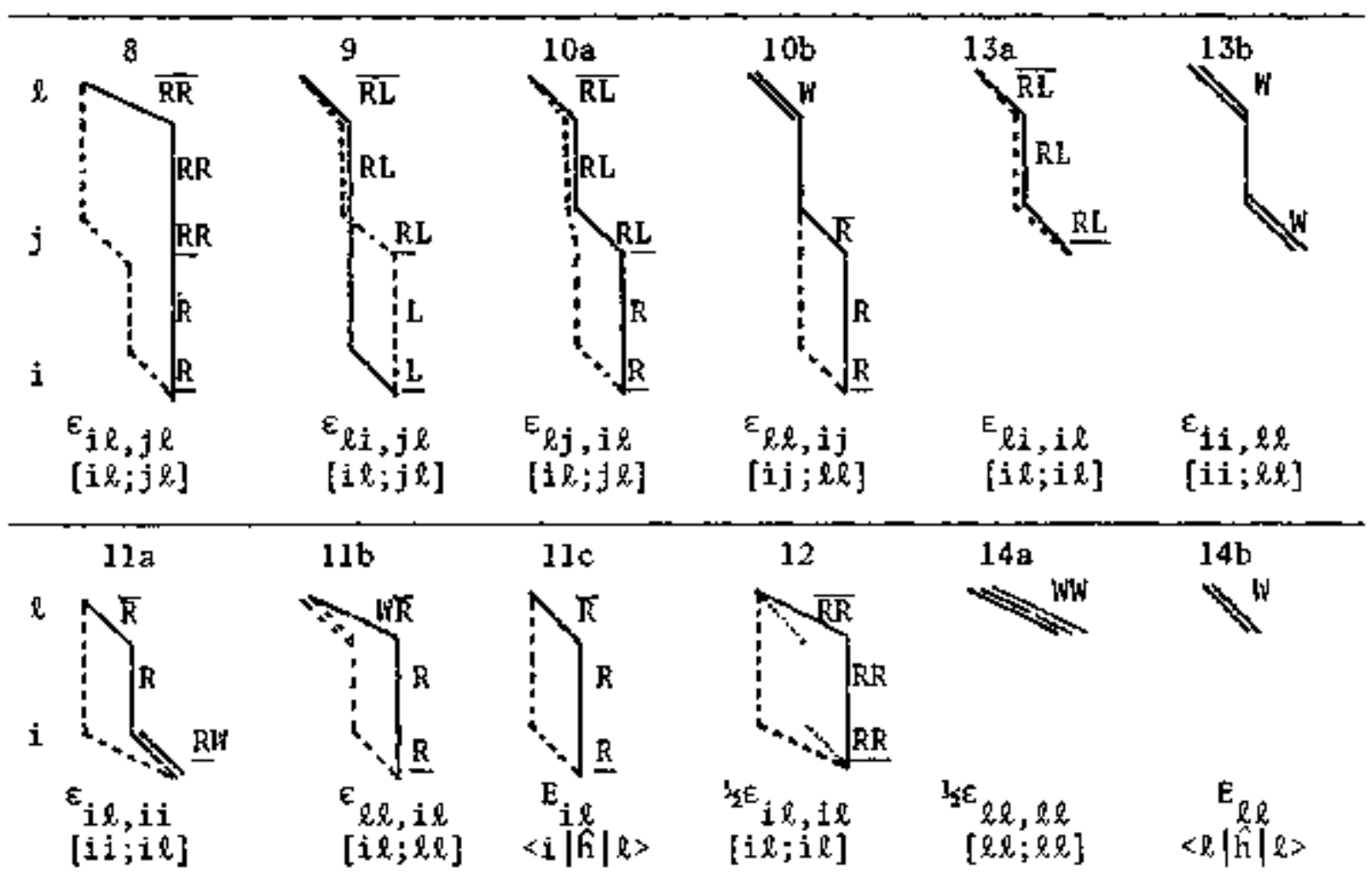


Figure 4.

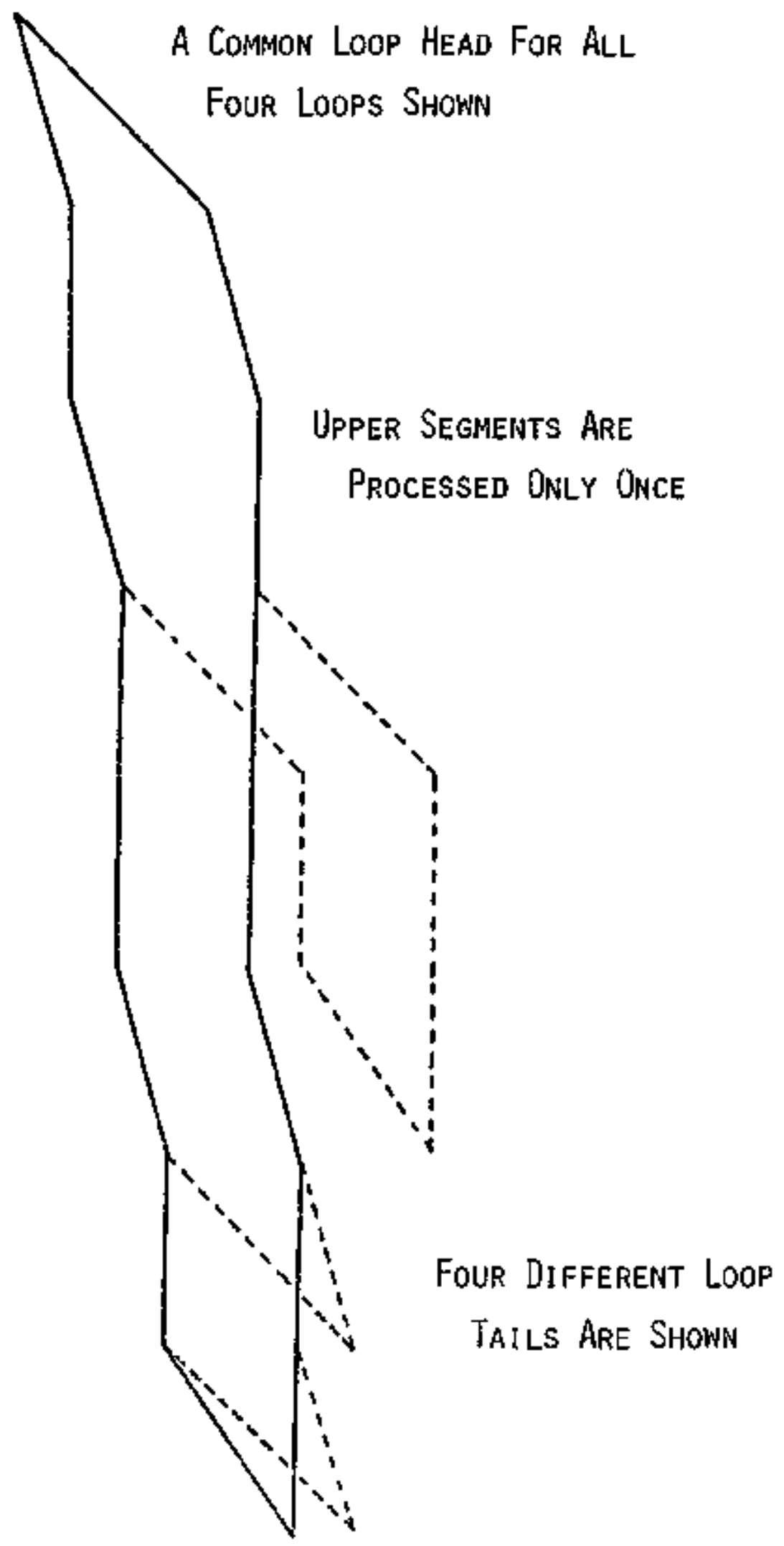


Figure 5.

\section{DIAGONALIZATION TAPE PROGRAM}

\begin{tabular}{|c|c|c|}
\hline A & 0.27 & $\begin{array}{l}\text { Generation of alstinct row table, } \\
\text { setup of arrays }\end{array}$ \\
\hline B & 0.45 & First half of integral sort \\
\hline C & 0.10 & Second half of integral sort \\
\hline D & 0.50 & Searching for new segments \\
\hline $\mathbf{E}$ & 0.98 & Processing accepted segments \\
\hline$F$ & 0.41 & Processing generated loops \\
\hline G & 0.38 & Pack loops into buffers \\
\hline
\end{tabular}

DIAGONALIZATION PROGRAM

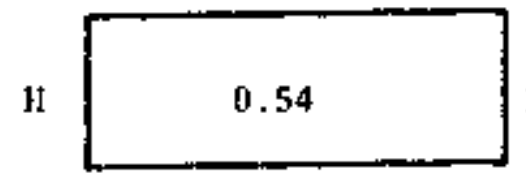

Each iteration of the diagonalization 
Figure 6. The BUGACI Systen of Programs

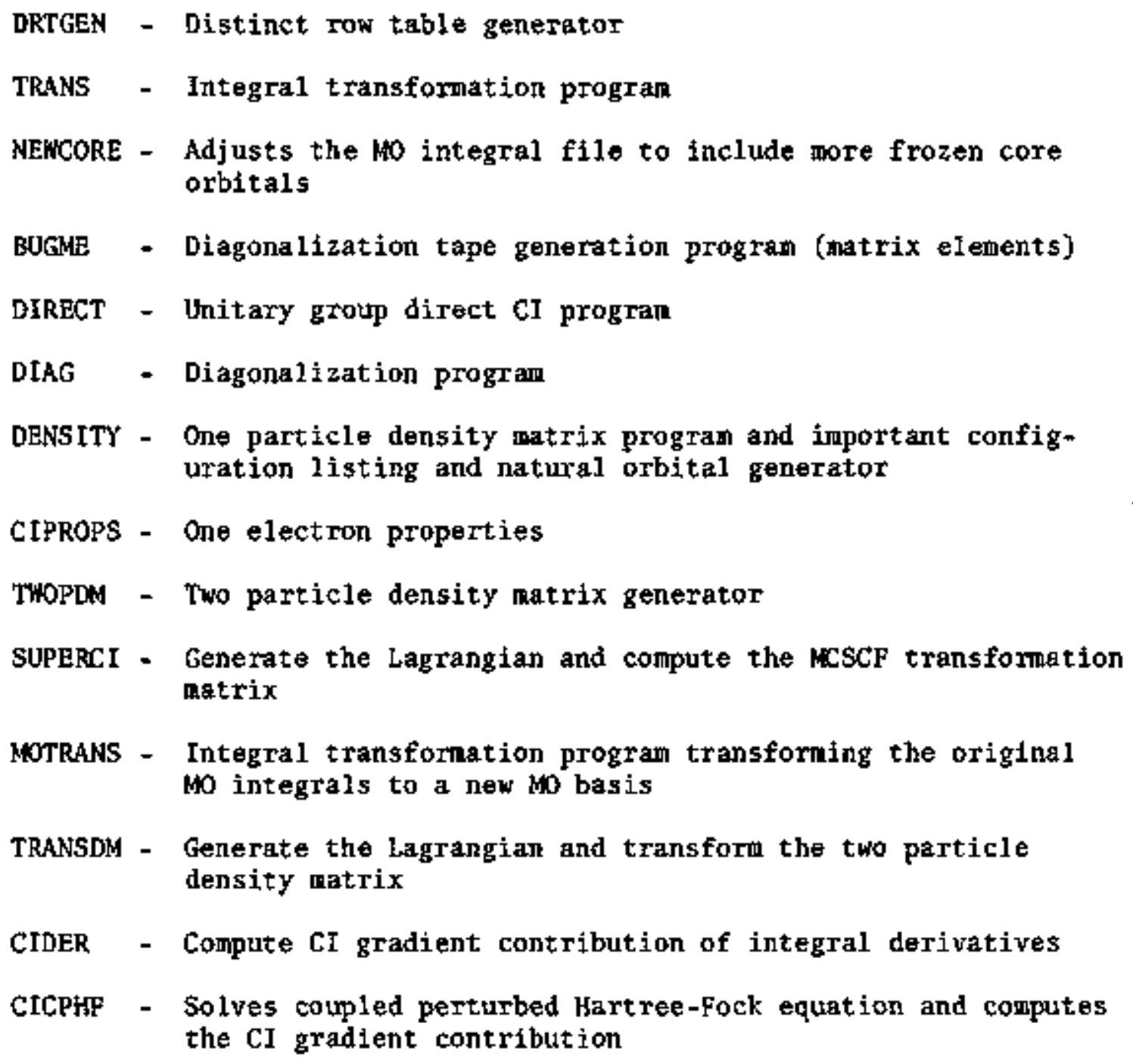




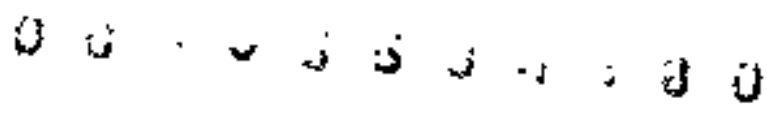

The BUGACl System of Programs

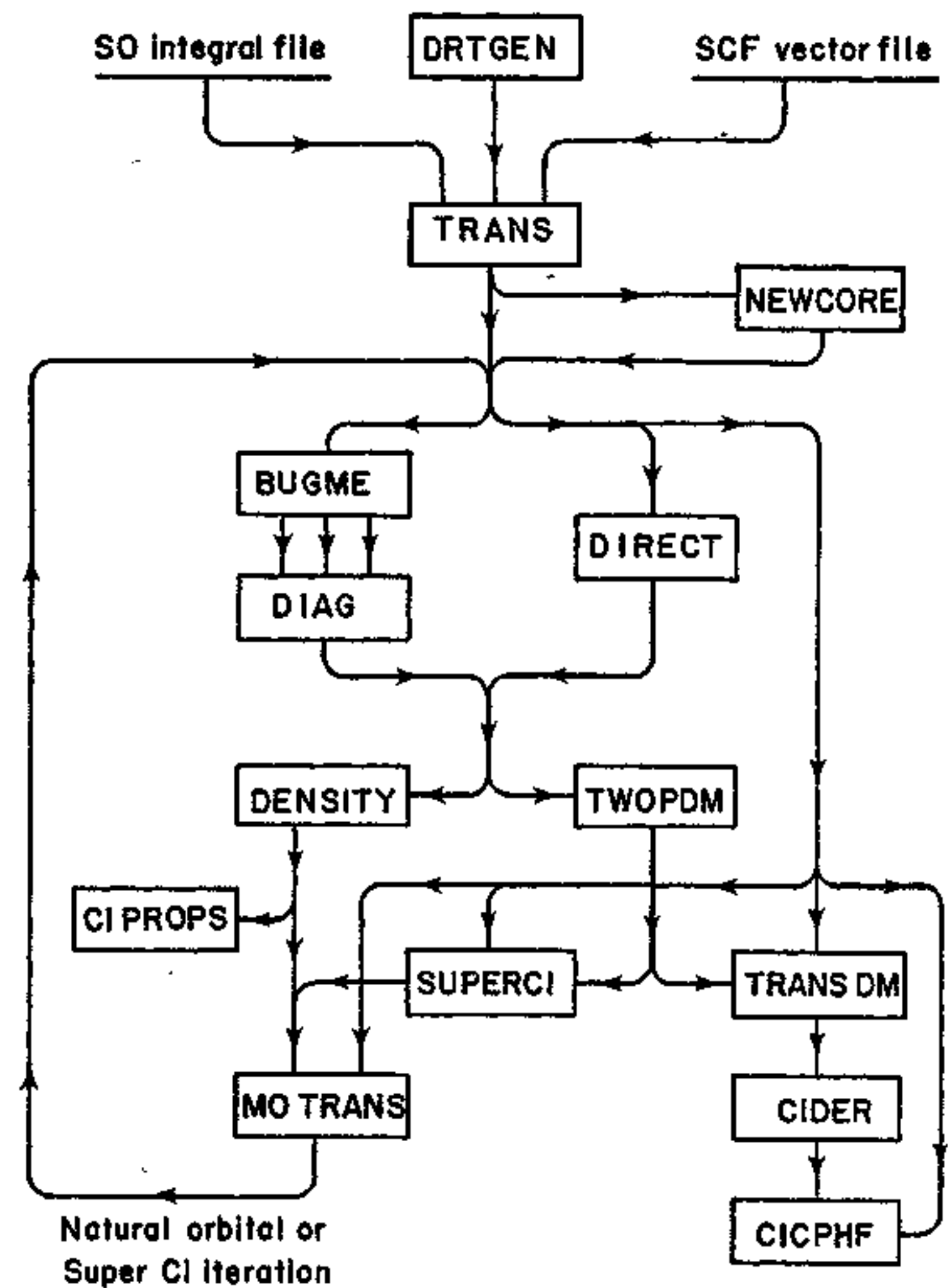


Example I. $\mathrm{BH}_{3}$ Ground State $\mathrm{C}_{2 \mathrm{v}}$ Subgroup

Extended basis get, 34 basis functions, 2355 configurations

$\begin{array}{ll}\text { Integrals over basis functions } & 9.01 \text { win } \\ \text { SCF (11 Iterations) } & 6.23 \text { min } \\ \text { Integral Transformation } & 9.08 \text { min }\end{array}$

Timing Comparisons: 
Example II. $\mathrm{CH}_{2}\left(\mathrm{C}_{1}\right) \mathrm{C}_{2 \mathrm{~V}}$ Symmetry

$$
1 a_{1}^{2} 2 a_{1}^{2} 3 a_{1}^{2} 1 b_{2}^{2}
$$

Extended basis set, 61 basis functions

(58 molecular orbitala), 7310 configurations

$$
\text { Integral Transformation } \quad 174.32 \text { nin }
$$

Timing Comparisons:

\begin{tabular}{lccc|c} 
& $\begin{array}{c}\text { Formula Tape } \\
\text { Generation }\end{array}$ & $\begin{array}{c}\text { Diagonalization } \\
\text { Tape Construction }\end{array}$ & Diagonalization & $\begin{array}{c}\text { Totals for One } \\
\text { Calculation }\end{array}$ \\
\hline $\begin{array}{l}\text { Integral Driven } \\
\text { Direct CI }\end{array}$ & No Formula Tape & 89.72 min & 51.02 min & 140.74 min \\
$\begin{array}{l}\text { Pretiminary GUGA } \\
\begin{array}{l}\text { Current Vergion } \\
\text { of GUGA }\end{array}\end{array}$ No Formula Tape & 14.00 min & $25.95 \mathrm{~min}$ & $69.13 \mathrm{~min}$ \\
\hline
\end{tabular}

Timing Breakdown of Current Version:

Distinct row table generation

Integral sorting

Loop generation (with implicit extecoal)

Loop generation (purely loop-driven)

Matrix mutiply each diagonalization Iteration
81.0 seconds

379.2 seconds

966.8 seconds

1066.8 secotds

219.7 seconds 
Example III. $\mathrm{C}_{2} \mathrm{H}_{4}{ }^{1_{B}}{ }_{1 u}$ (V state) $\mathrm{D}_{2 \mathrm{~h}}$ Symmetry

3 open shell singlet reference configurations

$$
\begin{aligned}
& 2 s_{g}^{2} 2 b_{1 u}^{2} 1 b_{l u}^{2} 3 e_{8}^{2} 1 b_{3 g}^{2} 1 b_{3 v} 1 b_{2 g} \\
& { }^{1 b_{3}}{ }^{2 b_{2}} \\
& { }^{1 \mathrm{~b}} 3 \mathrm{u}^{3 \mathrm{~b}_{2} \mathrm{~g}}
\end{aligned}
$$

Double zeta plus polarization and diffuse functions basis set, 44 basis functions ( 36 molecular orbitals included in the CI) 7022 configurations (interacting space on1y)

\begin{tabular}{|c|c|c|c|c|}
\hline & $\begin{array}{c}\text { Formula Tape } \\
\text { Generation }\end{array}$ & $\begin{array}{l}\text { Diagonalization } \\
\text { Tape Construction }\end{array}$ & Diagonal1zation & $\begin{array}{l}\text { Totals for One } \\
\text { Calculation }\end{array}$ \\
\hline Conventional CI & $211.9 \mathrm{~min}$ & 17.4 wisn & $16.9 \mathrm{ufn}$ & תin \\
\hline Preliminary GUGA & 18.9 min & 4.2 nin & 14.2 वain & $37.3 \mathrm{~min}$ \\
\hline $\begin{array}{l}\text { Current Version } \\
\text { of GUGA }\end{array}$ & No Formula Tape & 10.2 min & $14.1 \mathrm{~min}$ & $24.3 \mathrm{~min}$ \\
\hline
\end{tabular}

T1ming Comparisons:

$$
\text { Integral Transfornation } 28.31 \text { min }
$$

Timing Breakdown of Curreot Version:

D1stinct row table generation

Integral sort 1ng

Loop generation (with impilctt extemaI)

Loop generation (purely loop-driven)

Matrix multip1y each diagonalization iteration
73.1 seconds

46. I geconds

493.6 seconds

501.0 seconds

74.8 seconds 


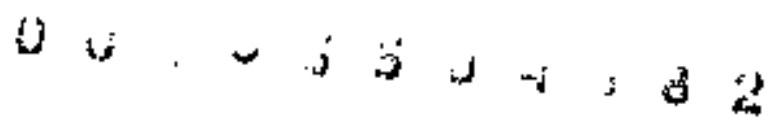

Examplo IV, $\mathrm{SO}_{2} 2^{1} A^{\prime} \mathrm{C}_{\mathrm{s}}$ symuetry

3 single reference configurations

$$
\begin{array}{r}
7 a^{2} 8 a^{2} 9 a^{2} 10 a^{2} 11 a^{2} 12 a^{2} 13 a a^{2} 2 a{ }^{2} 3 a^{\prime 2} \\
3 a^{\prime \prime} 4 a^{\prime \prime} \\
4 a^{\prime 2}
\end{array}
$$

Double zeta plus sulfur d function basis set; 44 basis functions (29 orbitals included in the $\mathrm{CI}$ ) 23,613 configurations (interacting space only)

Integral Transformation

Diagonalization Tape Construction

Sorting Diagonalization to Forr a Partitloned Hamiltonian

Diagonalization (9 iterations)
77.96 min

38.59 min

$57.51 \mathrm{inin}$

$164.39 \mathrm{~min}$ 
Table I. A snall sample distinct row table (DRT). $n=4, N=3, S=k$

\begin{tabular}{lllllllllllllll}
$\mathrm{i}$ & $\mathrm{j}$ & $\mathrm{a}$ & $\mathrm{b}$ & $\mathrm{c}$ & $\mathrm{k}_{0} \mathrm{k}_{1} \mathrm{k}_{2} \mathrm{k}_{3}$ & $y_{0} y_{1} y_{2}$ & $y_{3}$ & $\mathrm{x}$ & $\mathrm{x}$ \\
\hline 4 & 1 & 1 & 1 & 2 & 1 & 2 & 3 & 4 & 0 & 8 & 14 & 17 & 20 & 1 \\
3 & 1 & 1 & 1 & 1 & 1 & 2 & 3 & 4 & 0 & 2 & 5 & 6 & 8 & 1 \\
& 2 & 1 & 0 & 2 & 2 & - & 4 & 5 & 0 & - & 3 & 5 & 6 & 1 \\
& 3 & 0 & 2 & 1 & 3 & 4 & - & - & 0 & 1 & - & - & 3 & 1 \\
& 4 & 0 & 1 & 2 & 4 & 5 & - & - & 0 & 2 & - & - & 3 & 1 \\
2 & 1 & 1 & 1 & 0 & - & 1 & - & 2 & - & 0 & - & 1 & 2 & 1 \\
& 2 & 1 & 0 & 1 & 1 & - & 2 & 3 & 0 & - & 1 & 2 & 3 & 2 \\
& 3 & 0 & 2 & 0 & - & 2 & - & - & - & 0 & - & - & 1 & 2 \\
& 4 & 0 & 1 & 1 & 2 & 3 & - & - & 0 & 1 & - & - & 2 & 4 \\
& 5 & 0 & 0 & 2 & 3 & - & - & - & 0 & - & - & - & 1 & 2 \\
1 & 1 & 1 & 0 & 0 & - & - & - & 1 & - & - & - & 0 & 1 & 3 \\
& 2 & 0 & 1 & 0 & - & 1 & - & - & - & 0 & - & - & 1 & 9 \\
& 3 & 0 & 0 & 1 & 1 & $*$ & - & - & 0 & - & - & - & 1 & 8 \\
0 & 1 & 0 & 0 & 0 & - & - & - & - & - & - & - & - & 1 & 20 \\
\hline
\end{tabular}

${ }^{\mathrm{a}}$ Reference 24 . 


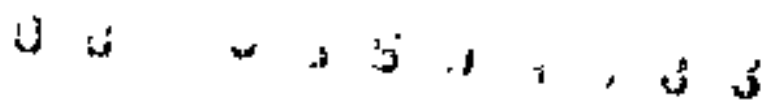

Table II. Coefficients of the five $S=1 / 2$ Gelfand states (configura tions) represented by Paldus tableaux expressed over slater deterninants for five unpaired electrons.

$$
[\mathrm{p}]=\left[\begin{array}{lll}
\underline{2} & 1 & 2 \\
2 & 0 & 2 \\
1 & 1 & 1 \\
1 & 0 & 1 \\
0 & 1 & 0 \\
0 & 0 & 0
\end{array}\right],\left[\begin{array}{lll}
\underline{2}^{*} & 1 & 2 \\
2 & 0 & 2 \\
1 & 1 & 1 \\
0 & 2 & 0 \\
0 & 1 & 0 \\
0 & 0 & 0
\end{array}\right],\left[\begin{array}{lll}
2 & \underline{3} \\
2 & 2 & 2 \\
1 & 1 & 1 \\
1 & 1 & 1 \\
1 & 0 & 1 \\
0 & 1 & 0 \\
0 & 0 & 0
\end{array}\right],\left[\begin{array}{lll}
2 & 1 & 2 \\
1 & 2 & 1 \\
1 & 1 & 1 \\
0 & 2 & 0 \\
0 & 1 & 0 \\
0 & 0 & 0
\end{array}\right],\left[\begin{array}{lll}
2 & 1 & 2 \\
1 & 2 & 1 \\
0 & 3 & 0 \\
0 & 2 & 0 \\
0 & 1 & 0 \\
0 & 0 & 0
\end{array}\right]
$$

\begin{tabular}{|c|c|c|c|c|c|}
\hline 1B $2 \beta \quad 3 \alpha \quad 4 \alpha \quad 5 \alpha$ & 0 & $-2 / \sqrt{12}$ & 0 & $1 / 3$ & $-1 / \sqrt{18}$ \\
\hline $1 \beta 2 \alpha 3 \beta \quad 4 \alpha 5 \alpha$ & $1 / 2$ & $1 / \sqrt{12}$ & $-1 / \sqrt{12}$ & $-1 / 6$ & $-1 / \sqrt{18}$ \\
\hline $1 \alpha 2 \beta \quad 3 \beta \quad 4 \alpha 5 \propto$ & $-1 / 2$ & $1 / \sqrt{12}$ & $1 / \sqrt{12}$ & $-1 / 6$ & $-1 / \sqrt{18}$ \\
\hline $182 \alpha 3 \alpha 4 \theta 5 \alpha$ & $-1 / 2$ & $1 / \sqrt{12}$ & $-1 / \sqrt{12}$ & $1 / 6$ & $1 / \sqrt{18}$ \\
\hline $1 \alpha 2 B \quad 3 \alpha 4 B \quad 5 \alpha$ & $1 / 2$ & $1 / \sqrt{12}$ & $1 / \sqrt{12}$ & $1 / 6$ & $1 / \sqrt{18}$ \\
\hline $2 \alpha 2 \alpha 3 \beta 4 \beta 5 \alpha$ & 0 & $-2 / \sqrt{\mathrm{I} 2}$ & 0 & $-1 / 3$ & $1 / \sqrt{18}$ \\
\hline $1 \beta 2 \alpha 3 \alpha 4 a 5 \beta$ & 0 & 0 & $2 / \sqrt{12}$ & $-1 / 3$ & $1 / \sqrt{18}$ \\
\hline $1 \alpha 2 \beta \quad 3 \alpha 4 \alpha 5 \beta$ & 0 & 0 & $-2 / \sqrt{12}$ & $-1 / 3$ & $1 / \sqrt{18}$ \\
\hline $1 \alpha 2 \alpha 3 \beta 4 \alpha 5 \beta$ & 0 & 0 & 0 & $2 / 3$ & $1 / \sqrt{18}$ \\
\hline $1 \propto 2 \alpha 3 \alpha \quad 4 \beta \quad 5 \beta$ & $\theta$ & 0 & 0 & 0 & $-1 / \sqrt{2}$ \\
\hline
\end{tabular}

*Hartree-Fock interacting configurations 
Table III. A sample distinct row table (ORT) that describes the configurations of the Hartree-Fock interacting space from the two open-shell reference configurations:

$$
\begin{aligned}
& 1 a^{2} 2 a^{2} 3 a \quad 4 a \\
& 1 a^{2} 2 a^{2} \text { 3a } 5 a
\end{aligned}
$$

for a seven orbital calculation without spatial symetry.

i j a b \& $\quad$ T T $\quad \mathrm{T}^{\prime} \quad \mathrm{k}_{0} \mathrm{k}_{1} \mathrm{k}_{2} \mathrm{k}_{3} \quad \mathrm{x} \quad \overline{\mathrm{x}}$

$\begin{array}{llllllllllllllllllllllll}7 & 1 & 3 & 0 & 4 & 0 & 0 & 1 & - & 2 & 3 & 177 & \end{array}$

\begin{tabular}{|c|c|c|c|c|c|c|c|c|c|}
\hline $\begin{array}{r}1 \\
2 \\
3\end{array}$ & $\begin{array}{lll}3 & 0 & 3 \\
2 & 1 & 3 \\
2 & 0 & 4\end{array}$ & $\begin{array}{l}0 \\
0 \\
1\end{array}$ & $\begin{array}{l}0 \\
0 \\
1\end{array}$ & $\begin{array}{l}1 \\
2 \\
5\end{array}$ & $\overline{4}$ & 3 & & 6 & \\
\hline
\end{tabular}

Orbital 3a active in references

Orbital $4 a$ active in references

$\begin{array}{rllllllllllrl}5 & 1 & 3 & 0 & 2 & 0 & 0 & 1 & - & 2 & 4 & 18 & 1 \\ 2 & 2 & 1 & 2 & 0 & 0 & 2 & 3 & 5 & 6 & 39 & 1 \\ 3 & 2 & 1 & 2 & 0 & 1 & 2 & 4 & 5 & 7 & 21 & 1 \\ 4 & 2 & 0 & 3 & 0 & 1 & 3 & - & 6 & 8 & 28 & 1 \\ 5 & 2 & 0 & 3 & 1 & 1 & 4 & - & 6 & 8 & 18 & 1 \\ 6 & 2 & 0 & 3 & 1 & 2 & 4 & - & 7 & * & 7 & 1 \\ 7 & 1 & 2 & 2 & 1 & 1 & 5 & 6 & - & 9 & 15 & 1 \\ 8 & 1 & 1 & 3 & 1 & 2 & 6 & 8 & 9 & - & 14 & 2 \\ 9 & 1 & 0 & 4 & 2 & 3 & 8 & - & - & - & 3 & 1\end{array}$

\begin{tabular}{|c|c|c|c|c|c|c|}
\hline 41 & 301 & 0 & $-\quad-$ & - & 1 & 3 \\
\hline 2 & 211 & 0 & - & 2 & 3 & 10 \\
\hline 3 & 202 & 0 & 1 & 3 & 5 & 15 \\
\hline 4 & 202 & 1 & - & 4 & 6 & 5 \\
\hline 5 & 121 & I & - & - & 8 & 4 \\
\hline 6 & 112 & 1 & 4 & 8 & 9 & 10 \\
\hline 7 & 112 & 2 & -7 & - & 10 & 2 \\
\hline 8 & 103 & 2 & $7=$ & 10 & 11 & 3 \\
\hline 9 & 022 & 2 & -10 & $=$ & - & 1 \\
\hline
\end{tabular}

Orbital $5 a$ active in references 


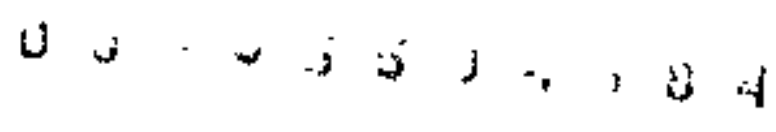

Table III, (continued)
i j
a b c $\quad T^{\prime} T^{\prime}$
$k_{0} k_{1} k_{2} k_{3}$
x $\bar{x}$

Orbital la doubly occupied in references

\begin{tabular}{|c|c|c|c|c|c|c|c|}
\hline 31 & 201 & 0 & - & - & $=$ & 1 & 3 \\
\hline 2 & 120 & 0 & - & - & - & 2 & 1 \\
\hline 3 & 111 & 0 & - & 1 & 2 & 3 & 6 \\
\hline 4 & 111 & 1 & - & - & - & 3 & 2 \\
\hline 5 & 102 & 0 & $I$ & - & 3 & 4 & 6 \\
\hline 6 & 102 & 1 & - & - & 3 & 4 & 3 \\
\hline 7 & 102 & 2 & - & - & - & 4 & 1 \\
\hline 8 & 021 & $I$ & . & 3 & - & - & 2 \\
\hline 9 & $\begin{array}{lll}0 & 1 & 2\end{array}$ & 1 & 3 & 4 & - & - & 3 \\
\hline 10 & 012 & 2 & - & 4 & - & - & 1 \\
\hline 11 & $\begin{array}{lll}0 & 0 & 3\end{array}$ & 2 & 4 & $=$ & - & - & 1 \\
\hline
\end{tabular}

Orbital 2 a doubly occupied in references

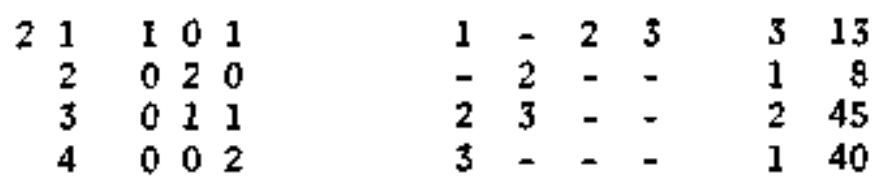

Orbital 6a unoccupied in references

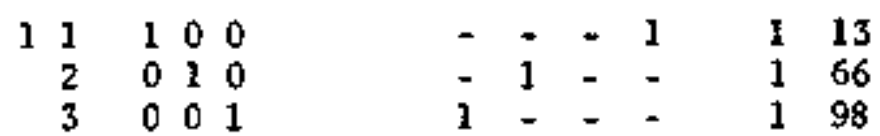

Orbital 7a unoccupied in references

$$
01000 \quad-\quad-\quad-\quad 1177
$$


Table IV. The Loop Searching Master Table

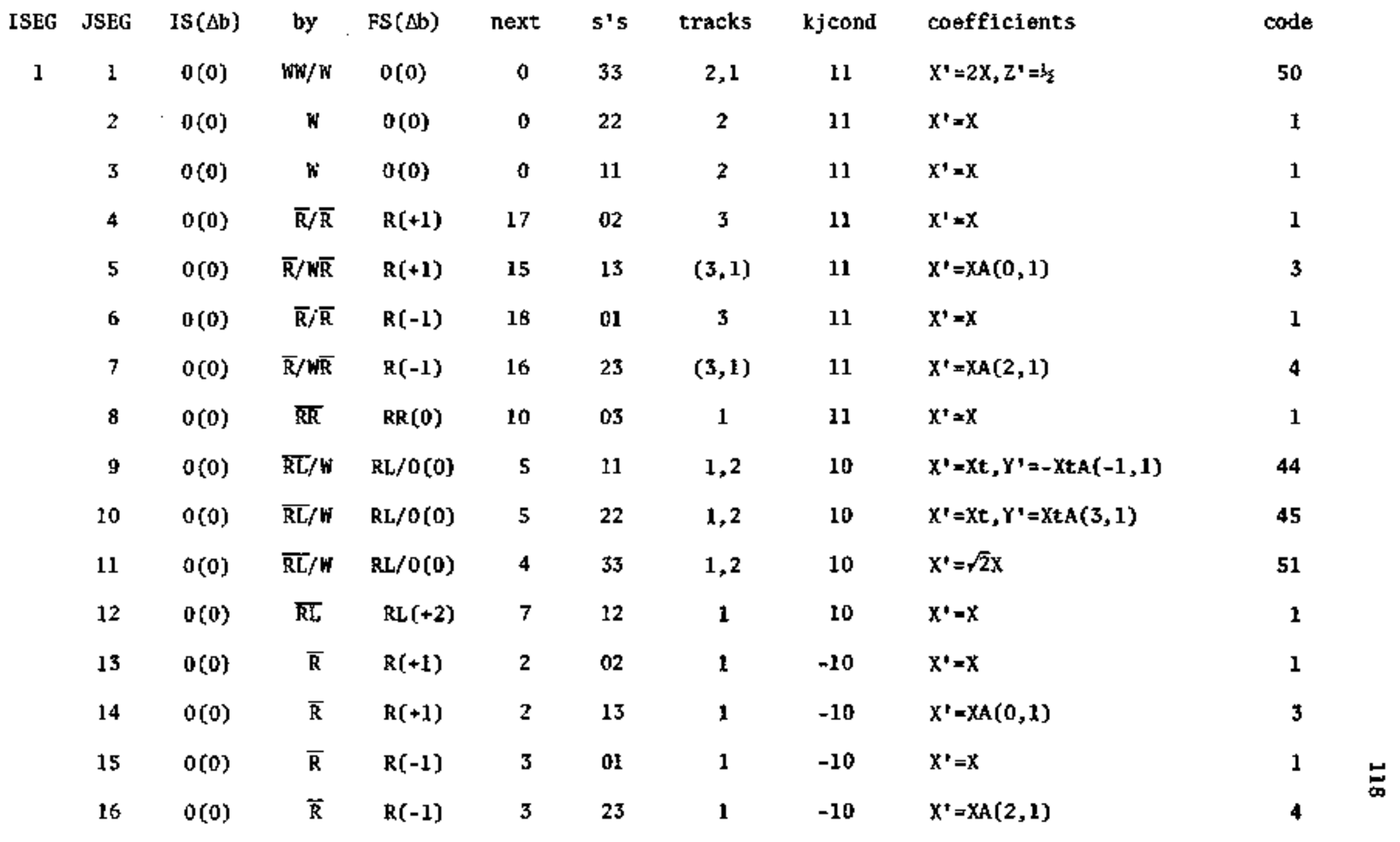




\begin{tabular}{|c|c|c|c|c|c|c|c|c|c|c|}
\hline ISEG & JSEG & IS $(\Delta b)$ & by & $F S(\Delta b)$ & next & $\mathbf{s}^{\prime} \mathbf{s}$ & tracks & kjcond & coefficients & code \\
\hline \multirow[t]{18}{*}{2} & 17 & $R(+1)$ & $\mathrm{R} \overline{\mathrm{L}}$ & $L(+1)$ & 20 & 30 & $\pi$ & 11 & $X^{\prime}=X A(1,0)$ & 5 \\
\hline & 18 & $R(+1)$ & $W R / \underline{R R}$ & $R(+1)$ & 21 & 22 & 2,1 & 11 & $X^{\prime}=-X, Z^{\prime}=-1$ & 52 \\
\hline & 19 & $R(+1)$ & WR/ $/ \bar{R}$ & $R(+1)$ & 21 & 33 & 2,1 & 11 & $X^{\prime}=-2 X, 2^{\prime}=-x_{2}$ & 53 \\
\hline & 20 & $R(+1)$ & WR & $\mathrm{R}(+1)$ & 21 & 11 & 2 & 11 & $x^{r}=x C(0)$ & 6 \\
\hline & 21 & $R\{+1\}$ & $\mathrm{WR} / \overline{\mathrm{R}} \underline{\underline{R}}$ & $R(-1)$ & 22 & 21 & 2,1 & 11 & $\mathrm{X}^{*}=\mathrm{X} / \mathrm{b}, \mathrm{Z}^{\prime}=\mathrm{b}$ & 54 \\
\hline & 22 & $R(+1)$ & $R \bar{R}$ & $R R(0)$ & 11 & 01 & 1,3 & 10 & $X^{\prime}=X t A(1,0), Y^{\prime}=-X t A(-1,0)$ & 40 \\
\hline & 23 & $R(+1)$ & $\mathbf{R} \overline{\mathbf{R}}$ & $\operatorname{RR}(0)$ & 11 & 23 & 1,3 & 10 & $X^{\prime}=-X t, Y^{1}=X t A(2,0)$ & 41 \\
\hline & 24 & $R(+1)$ & $\mathrm{R} \overline{\mathrm{R}}$ & $\mathrm{RR}(+2)$ & 12 & 02 & 1,3 & 10 & $X^{\prime}=x$ & 1 \\
\hline & 25 & $R(+1)$ & $\mathrm{R} \overline{\mathrm{R}}$ & $\mathrm{RR}(+2)$ & 12 & 13 & 1,3 & 10 & $X^{\prime}=X A(-1,0)$ & 7 \\
\hline & 26 & $R(+1)$ & $\mathbf{R} \overline{\mathbf{L}} / \underline{\mathbf{R}}$ & $\mathrm{RL} / 0(\mathrm{O})$ & 6 & 20 & 1,2 & 10 & $X^{\prime}=X t, Y^{\prime}=X t A(2,0)$ & 46 \\
\hline & 27 & $R(+1)$ & $\mathrm{R} \overline{\mathrm{L}} / \underline{\mathrm{R}}$ & $\mathrm{RL} / O(0)$ & 6 & 31 & 1,2 & 10 & $X^{1}=X t A(1,0), Y^{\prime}=X t A(-1,0)$ & 47 \\
\hline & 28 & $R(+1)$ & $\mathrm{R} \overline{\mathrm{L}}$ & $\mathbf{R L}(+2)$ & 7 & 10 & 3 & 10 & $x^{1}=x$ & 1 \\
\hline & 29 & $R(+1)$ & $\mathbf{R} \overline{\mathbf{L}}$ & $\mathrm{RL},(+2)$ & 7 & 32 & 3 & 10 & $X^{\prime}=-X A(1,0)$ & 8 \\
\hline & 30 & $R(\neq 1)$ & $\mathbf{R}$ & $R(+1)$ & 2 & 00 & - & -10 & $X^{\prime}=X$ & 1 \\
\hline & 31 & $R(+1)$ & $\mathbf{R}$ & $R(\div 1)$ & 2 & 11 & - & -10 & $X^{\prime}=X C(0)$ & 6 \\
\hline & 32 & $R(+1)$ & $\mathbf{R}$ & $R(+1)$ & 2 & 22 & - & -10 & $X^{1}=-X$ & 2 \\
\hline & 33 & $R(+1)$ & $\mathbf{R}$ & $R(+1)$ & 2 & 33 & r & -10 & $X^{\prime}=-X$ & 2 \\
\hline & 34 & $R(+1)$ & $\mathbf{R}$ & $R(-1)$ & 3 & 21 & - & -10 & $X^{\prime}=X / b$ & 9 \\
\hline
\end{tabular}

$c$

s.

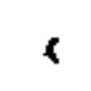

-

6.

i

-

t. 


\begin{tabular}{|c|c|c|c|c|c|c|c|c|c|c|}
\hline $\begin{array}{c}\text { ISEG } \\
3\end{array}$ & JSEG & $\operatorname{IS}(\Delta b)$ & by & $\mathrm{FS}(\Delta \mathrm{b})$ & next & s's & tracks & kjcond & coefficients & code \\
\hline \multirow[t]{18}{*}{3} & 35 & $R(-1)$ & $\underline{\mathrm{R}} \overline{\mathrm{L}}$ & $L(-1)$ & 19 & 30 & - & II & $x^{\prime}=x_{A}(1,2)$ & 10 \\
\hline & 36 & $R(-1)$ & $W R / \bar{R} \underline{R}$ & $R(-1)$ & 22 & 11 & 2,1 & 11 & $X^{\prime}=-X, Z^{*}=-1$ & 52 \\
\hline & 37 & $R(-1\}$ & $\mathrm{WR} / \overline{\mathrm{R}} \underline{\mathrm{R}}$ & $R(-1)$ & 22 & 33 & 2,1 & 11 & $X^{\prime}=-2 X, Z^{\prime}=-\mathrm{l}_{\mathbf{z}}$ & 53 \\
\hline & 38 & $\mathrm{R}(-1)$ & WR & $R(-1)$ & 22 & 22 & 2 & 11 & $X^{\prime}=X C(2)$ & 11 \\
\hline & 39 & $R(-1)$ & $\mathrm{WR} / \overline{\mathrm{R}} \underline{\mathrm{R}}$ & $R(+1)$ & 21 & 12 & 2,1 & 11 & $x^{\prime}=-x /(b+2), z^{t}=-(b+2)$ & 55 \\
\hline & 40 & $R\{* 1\}$ & $\mathbf{R} \bar{R}$ & $\operatorname{RR}\{0\}$ & 11 & 02 & 1,3 & 10 & $X^{\prime}=X t A(1,2), Y^{\prime}=X t A(3,2)$ & 42 \\
\hline & 41 & $R(-1)$ & $\mathbf{R} \overline{\mathbf{R}}$ & $\operatorname{RR}(0)$ & 11 & 13 & 1,3 & 10 & $X^{\prime}=-X t, Y^{\prime}=-X t A(0,2)$ & 43 \\
\hline & 42 & $\mathbf{R}(-1)$ & $\mathbf{R} \overline{\mathbf{R}}$ & $\operatorname{RR}(-2)$ & 13 & 01 & 1,3 & 10 & $x^{\prime}=x$ & 1 \\
\hline & 43 & $\mathrm{R}(-1)$ & $\mathbf{R} \overline{\mathbf{R}}$ & $\operatorname{RR}(-2)$ & 13 & 23 & 1,3 & 10 & $X^{\prime}=X A(3,2)$ & 12 \\
\hline & 44 & $R(-1)$ & $\mathrm{R} \overline{\mathrm{L}} / \underline{\mathbf{R}}$ & $\mathrm{RL} / 0\{0\}$ & 6 & 10 & 1,2 & 10 & $X^{\prime}=X t, Y^{1}=-X t A(0,2)$ & 48 \\
\hline & 45 & $R(-1)$ & $\mathrm{RL} / \underline{\mathrm{R}}$ & $\mathrm{RL} / 0(0)$ & 6 & 32 & 1,2 & 10 & $X^{\prime}=X \operatorname{XA}(1,2), Y^{+}+-X t A(3,2)$ & 49 \\
\hline & 46 & $R(-1)$ & $\mathbf{R} \overline{\mathbf{L}}$ & $\mathrm{RL}(-2)$ & 9 & 20 & 3 & 10 & $x^{\prime}=x$ & 1 \\
\hline & 47 & $R(-1)$ & $\mathbf{R} \mathbf{L}$ & $\operatorname{RL}(-2)$ & 9 & 31 & $\mathbf{3}$ & 10 & $X^{\prime}=-X A(1,2)$ & 13 \\
\hline & 48 & $R(-1)$ & $\mathbf{R}$ & $R(-1)$ & 3 & 00 & - & -10 & $x^{\prime}=x$ & 1 \\
\hline & 49 & $R(-1)$ & $\mathbf{R}$ & $\mathbf{R}\{-1)$ & 3 & $1 \mathbf{l}$ & - & -10 & $x^{\prime}=-x$ & 2 \\
\hline & 50 & $R(-1)$ & $\mathbf{R}$ & $R(-1)$ & 3 & 22 & - & -10 & $x^{\prime}=X C(2)$ & 11 \\
\hline & 51 & $R(-1)$ & $\mathbf{R}$ & $R(-1)$ & 3 & 33 & - & -10 & $x^{4}=-x$ & 2 \\
\hline & 52 & $R(-1)$ & $\mathbf{R}$ & $R(+1)$ & 2 & 12 & - & -10 & $\mathrm{X}^{\prime}=\mathrm{X} \mathrm{X} /(\mathrm{b}+2)$ & 36 \\
\hline
\end{tabular}




\begin{tabular}{|c|c|c|c|c|c|c|c|c|c|c|}
\hline ISEG & JSEG & IS $(\Delta b)$ & by & $\mathrm{FS}(\Delta \mathrm{b})$ & next & $s^{\prime} s$ & tracks & $\mathrm{kj}$ cond & coefficients & code \\
\hline \multirow[t]{11}{*}{4} & 53 & $\mathrm{RL} / 0(0)$ & $\underline{\mathrm{RL}} / W$ & $0(0)$ & 0 & 11 & $=$ & 01 & $X^{\prime}=-X t, z^{\prime}=-2$ & 77 \\
\hline & 54 & RL $/ 0(0)$ & $\underline{\mathrm{R} L} / \mathrm{W}$ & $0(0)$ & 0 & 22 & - & 01 & $X^{\prime}=-X t, Z^{\prime}=-2$ & 77 \\
\hline & 55 & $\mathrm{RL} / 0(0)$ & $\underline{\mathrm{RL}} / \mathrm{W}$ & $O(0)$ & 0 & 33 & - & 01 & $X^{\prime}=-\sqrt{2} X, Z^{1}=-2$ & 78 \\
\hline & 56 & RL $/ 0(0)$ & $\mathrm{Rl} / \mathrm{R}$ & $R(+1)$ & 21 & 02 & - & 01 & $X^{\prime}=-X t, Z^{\prime}=-2$ & 77 \\
\hline & 57 & $\mathrm{RL} / 0(0)$ & $\mathrm{RL} / \mathrm{R}$ & $R(+1)$ & 21 & 13 & - & 01 & $X^{\prime}=-X t A(0,1), Z^{r}=-2$ & 79 \\
\hline & 58 & $\mathrm{RL} / 0(0)$ & RL/R & $R(-1)$ & 22 & or & - & 01 & $X^{\prime}=-X t, Z^{\prime}=-2$ & 77 \\
\hline & 59 & $\mathrm{RL} / 0(0)$ & $\mathrm{RL} / \mathrm{R}$ & $R(-1)$ & 22 & 23 & - & 01 & $X^{\prime}=-X t A(2,1), Z^{\prime}=-2$ & 80 \\
\hline & 60 & $\mathrm{Rl} / 0(0)$ & $\mathrm{Rl} / 0$ & $\mathrm{RL} / 0(0)$ & 4 & 00 & - & 00 & $x^{\prime}=x$ & 1 \\
\hline & 61 & $\mathrm{RL} / 0(0)$ & $\mathrm{RL} / 0$ & RL/o(0) & 4 & I1 & - & 00 & $x^{*}=x$ & 1 \\
\hline & 62 & $\mathrm{Rl} / 0(0)$ & $\mathbf{R L} / 0$ & $\mathrm{RL} / 0(0)$ & 4 & 22 & - & 00 & $X^{\prime}=X$ & 1 \\
\hline & 63 & $\mathrm{RL} / 0(0)$ & $\mathrm{RL} / 0$ & $\mathrm{RL} / \mathrm{O}(0)$ & 4 & 33 & - & 00 & $X^{\prime}=X$ & 1 \\
\hline
\end{tabular}




\begin{tabular}{|c|c|c|c|c|c|c|c|c|c|c|}
\hline ISEG & JSEG & IS $(\Delta b)$ & by & $\mathrm{FS}(\Delta b)$ & next & s's & tracks & $\mathrm{kj}$ cond & coefficients & code \\
\hline \multirow[t]{12}{*}{5} & 64 & $\mathrm{RL} / 0(0)$ & $\underline{\mathrm{RL}} / \mathrm{W}$ & $0(0)$ & 0 & 11 & - & 01 & $\begin{array}{l}X^{+}=-X t+Y \operatorname{ta}(2,0)^{\dagger} \\
Z^{+}=\sqrt{2} X / X^{\prime}\end{array}$ & 87 \\
\hline & 65 & $\mathrm{RL} / \mathrm{O}(0)$ & $\underline{\mathrm{RL}} / \mathrm{W}$ & $0(0)$ & 0 & 22 & - & 01 & $\begin{array}{l}X^{\prime}=-X t-Y t A(0,2)^{\dagger} \\
Z^{\prime}=\sqrt{2 X} / X^{\prime}\end{array}$ & 68 \\
\hline & 66 & $\mathrm{RL} / 0(0)$ & $\underline{\mathbf{R} L / \mathrm{r}}$ & $0(0)$ & 0 & 33 & - & 01 & $x^{\prime}=-\sqrt{2} x, z^{\prime}=-2$ & 82 \\
\hline & 67 & $\mathrm{RL} / 0(0)$ & $\mathrm{R} \underline{\mathrm{L}} / \overline{\mathrm{R}}$ & $R\{+1\}$ & 21 & 02 & - & DI & $\begin{array}{l}X^{\prime}=-X t-Y \operatorname{ta}(0,2)^{\dagger} \\
Z^{\prime}=\sqrt{2} X / X^{1}\end{array}$ & 68 \\
\hline & 68 & $\mathrm{RL} / 0(0)$ & $\mathrm{R} \underline{\mathrm{L}} / \overline{\mathrm{R}}$ & $R(+1)$ & 21 & 13 & - & 01 & $\begin{array}{l}X^{\prime}=-X t A(0,1)-Y \operatorname{tA}(2,1)^{\dagger} \\
Z^{\prime}=\sqrt{2} X A(0,1) / X^{\prime}\end{array}$ & \\
\hline & 69 & $\mathrm{RL} / 0(0)$ & $\mathrm{R} \underline{\mathrm{L}} / \overline{\mathrm{R}}$ & $R(-1)$ & 23 & 01 & - & 01 & $\begin{array}{l}X^{\prime}=-X t+Y t A(2,0)^{\dagger} \\
Z^{\prime}=\sqrt{2} X / X^{+}\end{array}$ & 67 \\
\hline & 70 & $\mathrm{RL} / 0(0)$ & $\mathrm{R} \underline{\mathrm{L}} / \overline{\mathrm{R}}$ & $\mathrm{R}(-1)$ & 22 & 23 & - & 01 & $\begin{array}{l}X^{\prime}=-X t A(2,1)+Y_{t A}(0,1)^{\prime} \\
Z^{\prime}=\sqrt{2} X A(2,1) / X^{\prime}\end{array}$ & 70 \\
\hline & 71 & $\mathrm{RL} / \mathrm{O}(0)$ & $\mathrm{RL} / 0$ & $\mathrm{RL} / 0(0)$ & $\mathbf{5}$ & 00 & - & 00 & $X^{\prime}=X, Y^{*}=Y$ & 71 \\
\hline & 72 & $\mathrm{RL} / 0(0)$ & $\mathrm{RL} / 0$ & $\mathrm{RL} / \mathrm{O}(0)$ & 5 & 11 & - & 00 & $X^{t}=X, Y^{\prime}=Y D(0)$ & 75 \\
\hline & 73 & $\mathrm{RL} / 0(0)$ & $\mathrm{RL} / 0$ & $\mathrm{RL} / 0(0)$ & 5 & 22 & - & 00 & $X^{*}=X, Y^{*}=Y D(1)$ & 76 \\
\hline & 74 & $\mathrm{RL} / 0(0)$ & $\mathrm{RL} / 0$ & $\mathrm{RL} / 0(0)$ & 5 & 33 & - & 00 & $X^{\prime}=X, Y^{\prime}=Y$ & 71 \\
\hline & 75 & $R L(0)$ & RL & $\mathrm{RL}(+2)$ & 7 & 12 & 1 & 00 & $X^{\prime}=-Y E(0)$ & 83 \\
\hline
\end{tabular}

${ }^{\dagger}$ If $X^{\prime}=0$ transfer $\left(X^{\prime} Z^{\prime}\right)$ to $X^{\prime}$ and tracks to 2 


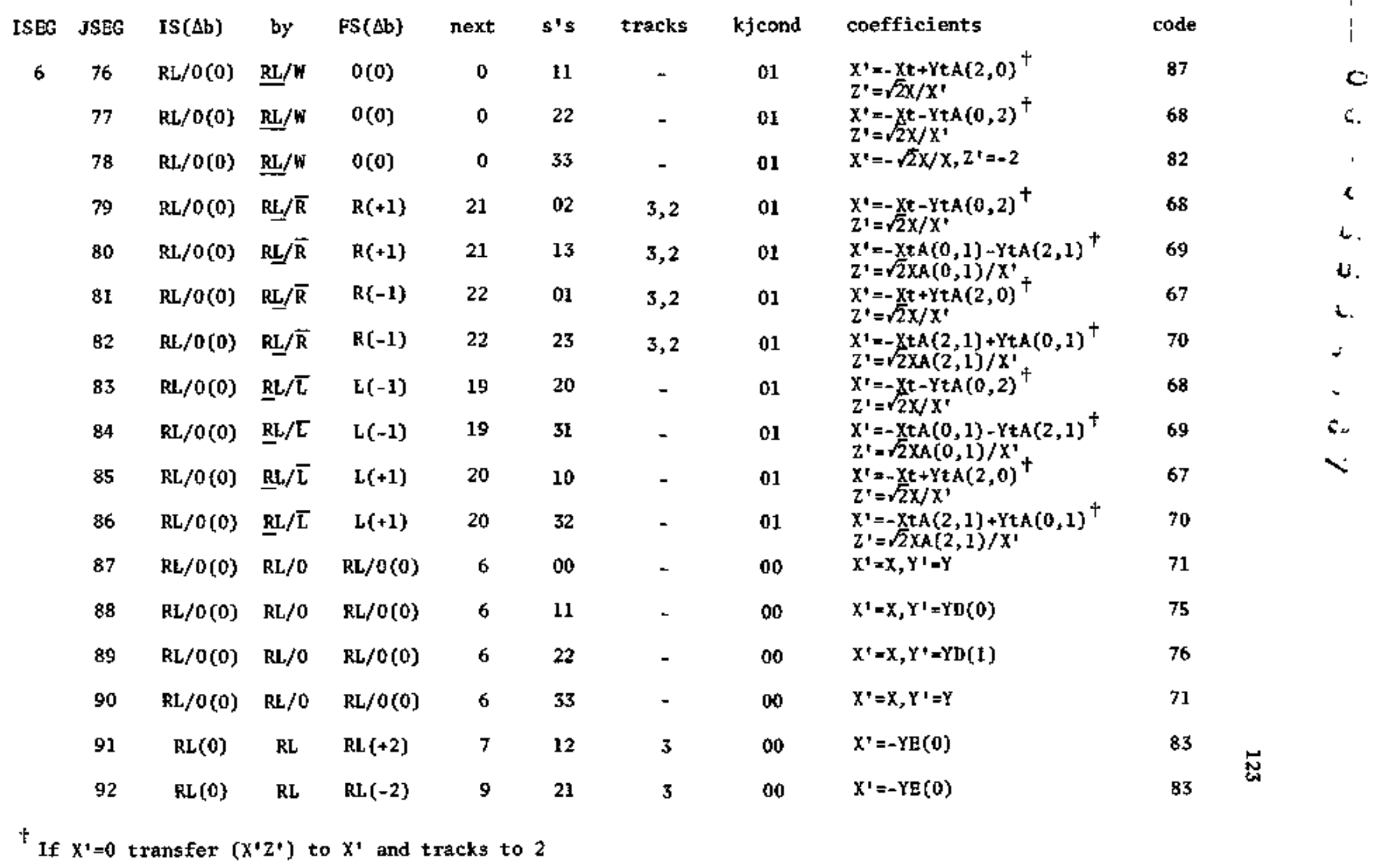




\begin{tabular}{|c|c|c|c|c|c|c|c|c|c|c|}
\hline ISEG & JSEG & $\operatorname{IS}(\Delta \mathrm{b})$ & by & $F S(\Delta b)$ & next & $\mathbf{s}^{\prime} \mathbf{s}$ & tracks & $\mathbf{x j}$ cond & coefficients & $\operatorname{cod}$ \\
\hline \multirow[t]{10}{*}{7} & 93 & $\operatorname{RL}(+2)$ & $\underline{\mathbf{R L}}$ & $o(0)$ & 0 & 21 & 1 & 01 & $x^{\prime}=X C(0)$ & 6 \\
\hline & 94 & $\operatorname{RL}(+2)$ & $\mathrm{R} \underline{\mathbf{L}}$ & $R(+1)$ & 22 & 01 & - & 01 & $x^{\prime}=x C(0)$ & 6 \\
\hline & 95 & $\mathrm{RL}(+2)$ & $\mathbf{R L}$ & $R(+1)$ & 21 & 23 & - & 01 & $X^{\prime}=-X A(-1,0)$ & 74 \\
\hline & 96 & $R L(+2)$ & $\underline{\mathrm{RL}}$ & $L(+1)$ & 20 & 20 & 1 & 01 & $x^{\prime}=x c(0)$ & \\
\hline & 97 & $R L(+2)$ & $\underline{\mathbf{R}} \mathbf{L}$ & $L(+1)$ & 20 & 31 & 1 & 01 & $X^{\prime}=-X A(1,0)$ & \\
\hline & 98 & $\operatorname{RL}(+2)$ & $\mathbf{R L}$ & $\mathrm{RL}(+2)$ & 7 & 00 & - & 00 & $x^{\prime}=x$ & \\
\hline & 99 & $\operatorname{RL}(+2)$ & $\mathbf{R L}$ & $R L(+2)$ & 7 & 11 & - & 00 & $X^{\prime}=-x C(0)$ & 10 \\
\hline & 100 & $\operatorname{RL}(+2)$ & $\mathbf{R l}$ & $R L(+2)$ & 7 & 22 & $=$ & 00 & $X^{\prime}=-x C(0)$ & \\
\hline & 101 & $R L(+2)$ & RL. & $\mathrm{RL}_{,}\{+2\}$ & 7 & 33 & - & 00 & $X^{+}=X$ & 1 \\
\hline & 102 & $\mathrm{RL}(+2)$ & $\mathbf{R L}$ & $R L(0)$ & 8 & 21 & - & 00 & $X^{1}=-X \sqrt{2} / b$ & \\
\hline
\end{tabular}




\begin{tabular}{|c|c|c|c|c|c|c|c|c|c|c|c|}
\hline ISEG & JSEG & $\operatorname{IS}(\Delta \mathrm{b})$ & by & $\mathrm{FS}(\Delta \mathrm{b})$ & next & $s^{\prime} s$ & tracks & kjcond & coefficients & code & \\
\hline \multirow[t]{16}{*}{8} & 103 & $R L(0)$ & $\underline{\mathbf{R L}}$ & $0(0)$ & 0 & 11 & 1 & 01 & $X^{\prime}=X t A(2,0)$ & 18 & $c$ \\
\hline & 104 & RL $(0)$ & $\underline{\mathrm{RL}}$ & $0(0)$ & 0 & 22 & 1 & 01 & $X^{\prime}=-X t A(0,2)$ & 19 & c. \\
\hline & 105 & $R L(0)$ & $\underline{R} \underline{L}$ & $R(+1)$ & 21 & 02 & $=$ & 01 & $X^{\prime}=-X t A(0,2)$ & 19 & \\
\hline & 106 & $R L(0)$ & RL & $R(+1)$ & 21 & 13 & $=$ & 01 & $x^{\prime}=-x t A(2,1)$ & 20 & $c$ \\
\hline & 107 & $\operatorname{RL}(0)$ & $\mathbf{R} \underline{\mathbf{L}}$ & $R\{-1\}$ & 22 & 01 & - & 01 & $x^{\prime}=x t A(2,0)$ & 18 & 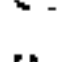 \\
\hline & 108 & $\operatorname{RL}(0)$ & $R \underline{L}$ & $R(-1)$ & 22 & 23 & - & 01 & $X^{\prime}=X t A(0,1)$ & 21 & 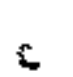 \\
\hline & 109 & $\mathrm{RL}(0)$ & $\underline{R L}$ & $L(-1)$ & 19 & 20 & 1 & 01 & $X^{\prime}=-X t A(0,2)$ & 19 & $\ldots$ \\
\hline & 110 & $R L(0)$ & $\underline{\mathbf{R} L}$ & $L(-1)$ & 19 & 31 & 1 & 01 & $X^{\prime}=-X \operatorname{xt}(2,1)$ & 20 & * \\
\hline & 111 & $\operatorname{RL}(0)$ & $\underline{\text { RL }}$ & $L(+1)$ & 20 & 10 & 1 & 01 & $X^{\prime}=X t A(2,0)$ & 18 & is \\
\hline & 112 & $\operatorname{RL}(0)$ & RL & $L(+1)$ & 20 & 32 & $I$ & 01 & $X^{1}=X t A(0,1)$ & 21 & Cier \\
\hline & 113 & RL $(0)$ & RL & RL(0) & 8 & DO & - & 00 & $X^{r}=x$ & 1 & \\
\hline & 114 & $\operatorname{RL}(0)$ & RL & $\mathbf{R L}(0)$ & 8 & 11 & - & 00 & $X^{s}=X D(0)$ & 22 & \\
\hline & 115 & $\operatorname{RL}(0)$ & Rl & $\operatorname{RL}(0)$ & 8 & 22 & - & 00 & $X^{\prime} \times X D(1)$ & 23 & \\
\hline & 116 & $\operatorname{Rl}(0)$ & RL & $\operatorname{RL}(0)$ & 8 & 33 & - & 00 & $X^{\prime}=X$ & I & \\
\hline & 117 & RL $(0)$ & RL & $\operatorname{RL}\{+2\}$ & 7 & 12 & - & 00 & $X^{\prime}=-X E(0)$ & 24 & \\
\hline & $\$ 18$ & $\operatorname{RL}(0)$ & RL & $\operatorname{RL}(-2)$ & 9 & 21 & - & 00 & $X^{\prime}=-X E(0)$ & 24 & \\
\hline
\end{tabular}




\begin{tabular}{|c|c|c|c|c|c|c|c|c|c|c|}
\hline ISEG & JSEG & IS ( $\Delta \mathrm{b})$ & by & $\operatorname{FS}(\Delta b)$ & next & s's & tracks & $\mathrm{kj} \cos d \mathbf{d}$ & coefficients & $\operatorname{cod}$ \\
\hline \multirow[t]{10}{*}{9} & 119 & $R L(-2)$ & $\underline{\mathrm{RL}}$ & $0(0)$ & 0 & 12 & 1 & 01 & $x^{\prime}=x C(2)$ & 11 \\
\hline & 120 & $\operatorname{RL}(-2)$ & RL & $R(-1)$ & 22 & 02 & - & 01 & $x^{\prime}=x c(2)$ & 11 \\
\hline & 121 & $\mathbf{R L}(-2)$ & RL & $\mathbb{R}(-1)$ & 22 & 13 & - & 01 & $x^{\prime}=-x_{A}(3,2)$ & 81 \\
\hline & 122 & $\mathrm{RL}(-2)$ & $\underline{\mathbf{R}} \mathbf{L}$ & $L(-1)$ & 19 & 10 & 1 & 01 & $X^{\prime}=x C(2)$ & 11 \\
\hline & 123 & $\operatorname{RL}(-2)$ & $\underline{\mathbf{R}} \mathbf{L}$ & $L(-1)$ & 19 & 32 & 1 & 01 & $X=-X A(1,2)$ & 13 \\
\hline & 124 & $R L(-2)$ & $\mathbf{R L}$ & $\operatorname{RL}\{-2\}$ & 9 & 00 & - & 00 & $x^{\prime}=x$ & 1 \\
\hline & 125 & $\operatorname{RL}(-2)$ & RL & $R L(-2)$ & $\mathbf{9}$ & 11 & - & 00 & $x^{\prime}=-x C(2)$ & 27 \\
\hline & 126 & $R L(-2)$ & RL & $R L(-2)$ & 9 & 22 & - & 00 & $x^{\prime}=-x c(2)$ & 27 \\
\hline & 127 & $\operatorname{RL}(-2)$ & RL & $\operatorname{RL}(-2)$ & 9 & 33 & - & 00 & $x^{\prime}=x$ & $\mathbf{1}$ \\
\hline & 128 & $\operatorname{RL}(-2)$ & RL & RL(0) & 8 & 12 & - & 00 & $X^{\prime}=-\sqrt{2} X /(b+2)$ & \\
\hline
\end{tabular}




\begin{tabular}{|c|c|c|c|c|c|c|c|c|c|}
\hline ISEG & JSEG & $I S(A b)$ & by & $F S(\Delta b)$ & next & s's & tracks & $\mathrm{kj}$ cond & coefficlents \\
\hline \multirow[t]{9}{*}{10} & 129 & RR(0) & $\underline{\mathbf{R R}}$ & $0(0)$ & 0 & 30 & - & 01 & $X^{1}=X$ \\
\hline & 130 & $\mathrm{RR}(0)$ & $\underline{\mathbf{R}} \underline{\underline{R}}$ & $R(+1)$ & 21 & 10 & - & 01 & $X^{+}=X A(0,1)$ \\
\hline & 131 & $\operatorname{RR}(0)$ & $\underline{\mathbf{R R}}$ & $R(+1)$ & 21 & 32 & - & OI & $x^{\prime}=-x$ \\
\hline & 132 & $\mathrm{RR}(0)$ & $\underline{\mathbf{R}} \mathbf{R}$ & $R(-1)$ & 22 & 20 & - & 01 & $X^{4}=X A(2,1)$ \\
\hline & 133 & $\mathrm{RR}(0)$ & $\underline{R R}$ & $R(-1\}$ & 22 & 31 & $=$ & 01 & $X^{\prime}=-X$ \\
\hline & 134 & RR (0) & RR & $\operatorname{RR}(0)$ & 10 & 00 & - & 00 & $X^{\prime}=X$ \\
\hline & 135 & $\mathrm{RR}(0)$ & RR & $\operatorname{RR}(0)$ & 10 & 11 & - & 00 & $X^{1}=-X$ \\
\hline & 136 & RR(0) & RR & $R R(0)$ & 10 & 22 & - & 00 & $x^{\prime}=-x$ \\
\hline & 137 & $\mathrm{RR}(0)$ & RR & $R R(0)$ & 10 & 33 & - & 00 & $X^{4}=x$ \\
\hline
\end{tabular}

code
1
3
2
4
2
1
2
2
1




\begin{tabular}{|c|c|c|c|c|c|c|c|c|c|}
\hline ISEG & JSEG & IS $(\Delta b)$ & by & $F S(\Delta b)$ & next & s's & tracks & kj cond & coefficients \\
\hline 11 & 138 & $\mathbf{R R}(0)$ & $\underline{\mathrm{RR}}$ & $0(0)$ & 0 & 30 & 1 & 01 & $X^{\prime}=\sqrt{2} X$ \\
\hline & 139 & $\operatorname{RR}(0)$ & $\underline{\mathrm{R}} / \mathrm{RR}^{\star}$ & $R(+1)$ & 21 & 10 & - & 01 & $\begin{array}{l}X^{\prime}=X t A(0,1)+Y t A(2,1)^{\dagger t} \\
Z^{\prime}=\{x \in A(0,1)-Y t A(2,1)\} X^{\circ}\end{array}$ \\
\hline & 140 & $\mathrm{RR}(0)$ & $\underline{R R} / \underline{R R}^{*}$ & $R(+1)$ & 21 & 32 & - & 01 & $\begin{array}{l}X^{\prime}=-X \operatorname{Xt}-Y t A(0,2) T^{\prime} \\
Z^{\prime}=[-X t+Y t A(0,2)) / X^{1}\end{array}$ \\
\hline & 141 & $\mathrm{RR}(0)$ & $\underline{\mathrm{RR}} / \mathrm{RR}^{*}$ & $R(-1)$ & 22 & 20 & - & 02 & $\begin{array}{l}X^{\prime}=X \operatorname{tA}(2,1)-Y t A(0,1)^{+\dagger} \\
Z^{\prime}=(X \pm A(2,1)+Y \operatorname{ta}(0,1)) / X^{\prime}\end{array}$ \\
\hline & 142 & $\operatorname{RR}(0)$ & $\underline{\mathrm{RR}} / \underline{\mathrm{RR}}^{*}$ & $R(-1)$ & 22 & 31 & - & 01 & $\begin{array}{l}X^{\prime}=-X t+Y t A(2,0) \\
Z^{\prime}=(-X t-Y t A(2,0)) / X^{\prime}\end{array}$ \\
\hline & 143 & $\operatorname{RR}(0)$ & RR & $\operatorname{RR}(0)$ & 11 & 00 & - & 00 & $X^{1}=X, Y^{\prime}=Y$ \\
\hline & 144 & $\operatorname{RR}(0)$ & $\mathbf{R R}$ & $\operatorname{RR}(0)$ & 11 & 11 & - & 00 & $X^{\prime}=-X, Y^{\prime}=-Y D(\theta)$ \\
\hline & 145 & $R R(0)$ & RR & $\mathrm{RR}(0)$ & 11 & 22 & - & 00 & $X^{\prime}=-X, Y^{\prime}=-Y D(1)$ \\
\hline & 146 & $\mathrm{RR}(0)$ & RR & $\mathrm{RR}(0)$ & I I & 33 & - & 00 & $X X^{\prime}=X, Y^{\prime}=Y$ \\
\hline & 147 & RR $(0)$ & $\mathrm{RR}$ & $\mathrm{RR}(+2)$ & 12 & 12 & - & 00 & $X^{\prime}=Y B(1)$ \\
\hline & 148 & $\operatorname{RR}(0)$ & $\mathbf{R R}$ & $R R[-2)$ & 13 & 21 & $=$ & 00 & $X^{\prime}=Y B(0)$ \\
\hline
\end{tabular}




\begin{tabular}{|c|c|c|c|c|c|c|c|c|c|c|}
\hline ISEG & ISEG & $\operatorname{IS}(\Delta b)$ & by & $\mathrm{FS}(\Delta \mathrm{b})$ & next & s's & tracks & $k j$ cond & coefficients & code \\
\hline \multirow[t]{7}{*}{12} & 149 & $\mathrm{RR}(+2)$ & $\underline{R R} / R^{*}$ & $R(+1)$ & $2 \mathrm{I}$ & 20 & - & 01 & $X^{\prime}=X, Z^{\prime}=-1$ & 56 \\
\hline & 150 & $\mathbf{R R}(+2)$ & $\underline{\mathrm{RR}} / \mathrm{RR}^{*}$ & $R(+1)$ & 21 & 31 & - & 01 & $X^{1}-X A(1,0), 2^{\prime}=-1$ & 57 \\
\hline & 151 & $\mathrm{RR}(+2)$ & $\mathbf{R R}$ & $\mathrm{RR}(+2)$ & 12 & 00 & - & 00 & $x^{1}=x$ & 1 \\
\hline & 152 & $\mathrm{RR}(+2)$ & $\mathbf{R R}$ & $\mathrm{RR}[+2)$ & 12 & 11 & - & 00 & $\mathrm{X}^{\prime}=\mathrm{XD}(-1)$ & 30 \\
\hline & 153 & $R R(+2)$ & $\mathbf{R R}$ & $\mathrm{RR}(+2)$ & 12 & 22 & - & 00 & $\mathrm{X}^{ \pm}=\mathrm{X}$ & I \\
\hline & 154 & $\operatorname{RR}(+2)$ & $\mathbf{R R}$ & $\mathrm{RR}(+2)$ & 12 & 33 & - & 00 & $X^{\prime}=X$ & 1 \\
\hline & 155 & $\operatorname{RR}(+2)$ & RR & $\operatorname{RR}(0)$ & 14 & 21 & - & 00 & $X^{\prime}=X B(-1)$ & 86 \\
\hline
\end{tabular}




\begin{tabular}{|c|c|c|c|c|c|c|c|c|c|c|}
\hline ISEG & JSEG & $\operatorname{IS}(\Delta \mathrm{b})$ & by & $\mathrm{FS}(\Delta \mathrm{b})$ & next & $s^{\prime} s$ & tracks & $\mathrm{kj}$ cond & coefficients & code \\
\hline \multirow[t]{7}{*}{13} & 156 & $R R(-2)$ & $\underline{\mathrm{RR}} / \mathrm{RR}^{*}$ & $R(-1)$ & 22 & 10 & - & 01 & $x^{r}=X, 2^{\prime}=-1$ & 56 \\
\hline & 157 & $R R(-2)$ & $\underline{\mathrm{R} R} / \mathrm{RR}^{*}$ & $R(-1)$ & 22 & 32 & $=$ & 01 & $X^{\prime}=X A(1,2), Z^{+}=-1$ & 58 \\
\hline & 158 & $\operatorname{RR}(-2)$ & $\mathbf{R R}$ & $R R(-2)$ & 13 & 00 & - & 00 & $X^{\prime}=X$ & 1 \\
\hline & 159 & $R R(-2)$ & $\mathbf{R R}$ & $\operatorname{RR}(-2)$ & 13 & 11 & $=$ & 00 & $X^{\prime}=X$ & 1 \\
\hline & 160 & $R R(-2)$ & $\mathbf{R R}$ & $\operatorname{RR}(-2)$ & 13 & 22 & - & 00 & $X^{\prime}=X D(2)$ & 31 \\
\hline & 161 & $R R(-2)$ & RR & $\operatorname{RR}(-2)$ & 13 & 33 & - & 00 & $x^{\prime}=x$ & 1 \\
\hline & 162 & $R R(-2)$ & $\mathbf{R R}$ & $R R(0)$ & 14 & 12 & - & 00 & $X^{1}=X B(2)$ & 32 \\
\hline
\end{tabular}




\begin{tabular}{|c|c|c|c|c|c|c|c|c|c|c|}
\hline ISEG & JSEG & IS $(A b)$ & by & $\mathbf{E S}(\Delta \mathrm{b})$ & next & $s^{1} s$ & tracks & $x j \operatorname{cond}$ & coefficients & cade \\
\hline \multirow[t]{10}{*}{14} & 163 & $\mathrm{RR}(0)$ & $\underline{\mathrm{RR}} / \mathrm{RR}^{*}$ & $\mathbf{R}(+1)$ & 21 & 10 & - & 01 & $X^{\prime}=X \operatorname{At}(2,1), Z^{\prime}=-1$ & 59 \\
\hline & 164 & $\operatorname{RR}(0)$ & $\underline{\mathrm{RR}} / \mathrm{RR}^{*}$ & $R(+1)$ & 21 & 32 & - & 01 & $X^{\prime}=-X t A(0,2), Z^{\prime}=-1$ & 60 \\
\hline & 165 & $\mathrm{RR}(\theta)$ & $\underline{\mathrm{RR}} / \mathrm{RR}^{*}$ & $\mathbf{R}(-1)$ & 22 & 20 & - & 01 & $X^{\prime}=-X \operatorname{tA}(0,1), Z^{\prime}=-1$ & 61 \\
\hline & 166 & $\mathrm{RR}(0)$ & $\underline{\mathrm{RR}} / \underline{\mathrm{RR}}^{*}$ & $R(-1)$ & 22 & 31 & - & 01 & $x^{\prime}=x \operatorname{ta}(2,0), Z^{\prime}=-1$ & 62 \\
\hline & 167 & $\mathrm{RR}(0)$ & RR & $\mathrm{RR}(0)$ & 14 & 00 & - & 00 & $x^{\prime}=X$ & $\mathbf{I}$ \\
\hline & 168 & $\mathrm{RR}(0)$ & $\mathbf{R R}$ & $\mathrm{RR}(0)$ & 14 & 11 & - & 00 & $X^{\prime}=-X D(0)$ & $\mathbf{3 3}$ \\
\hline & 169 & $\mathrm{RR}(0)$ & $\mathbf{R R}$ & $\mathrm{RR}(0)$ & 14 & 22 & - & 00 & $x^{\prime}=x D(-1)$ & 30 \\
\hline & 170 & $\operatorname{RR}(0)$ & RR & $\operatorname{RR}(0)$ & 14 & 33 & - & 00 & $x^{\prime}=x$ & 1 \\
\hline & 171 & $R R(0)$ & $\mathbf{R R}$ & $\mathrm{RR}(+2)$ & 12 & 12 & - & 00 & $X^{\prime}=X B(1)$ & 34 \\
\hline & 172 & $\mathrm{RR}(0)$ & $\mathrm{RR}$ & $R R(-2)$ & 13 & 21 & - & 00 & $X^{1}=X B(0)$ & 35 \\
\hline
\end{tabular}




\begin{tabular}{|c|c|c|c|c|c|c|c|c|c|c|}
\hline ISEG & JSEG & IS $(\Delta \mathrm{b})$ & by & $F S(\Delta b)$ & next & s's & tracks & $k j$ cond & coefficients & code \\
\hline \multirow[t]{7}{*}{15} & 173 & $R(+1)$ & $\underline{\mathbf{R}}$ & $0(0)$ & 0 & 20 & - & 00 & $X^{\prime}=X$ & 1 \\
\hline & 374 & $R(\div 1)$ & $\underline{\mathrm{R}} / \mathrm{W}$ & $0(0)$ & 0 & 31 & $(3,2,1)$ & 00 & $X^{\prime}=X A(1,0)$ & 5 \\
\hline & 175 & $R(+1)$ & $\mathbf{R}$ & $R(+1)$ & 15 & 00 & - & $\infty 0$ & $X^{\prime}=\mathrm{X}$ & 1 \\
\hline & 176 & $R(+1)$ & $\mathbf{R}$ & $R(+1)$ & 15 & 11 & - & 00 & $X^{2}=x C(0)$ & 6 \\
\hline & 177 & $R(+1)$ & $\mathbf{R}$ & $\mathrm{R}(+1)$ & 15 & 22 & - & 00 & $x^{\prime}=-x$ & 2 \\
\hline & 178 & $R(+1)$ & $\mathbf{R}$ & $R(+1)$ & 15 & 33 & - & 00 & $X^{\prime}=-X$ & 2 \\
\hline & 179 & $R(+1)$ & $\mathrm{R}$ & $R(-1)$ & 16 & 21 & - & 00 & $X^{\prime}=X / b$ & 9 \\
\hline \multirow[t]{7}{*}{16} & 180 & $R(-1)$ & $\underline{\mathrm{R}}$ & $0(0)$ & 0 & 10 & - & 00 & $x^{\prime}=x$ & 1 \\
\hline & 181 & $R(-1)$ & $\underline{\mathrm{R}} / \mathrm{WR}$ & $o(0)$ & 0 & 32 & $(3,2,1)$ & $\infty$ & $X^{\prime}=X A(1,2)$ & 10 \\
\hline & 182 & $R(-1)$ & $\mathbf{R}$ & $\mathbf{R}(-\mathbf{I})$ & 16 & 00 & - & 00 & $x^{\prime}=x$ & 1 \\
\hline & 183 & $R(-1)$ & $\mathbf{R}$ & $R(-1)$ & 16 & 11 & - & 00 & $x^{\prime}=-x$ & 2 \\
\hline & 184 & $R(-1)$ & $\mathbf{R}$ & $R(-1)$ & 16 & 22 & - & 00 & $x^{\prime}=x C(2)$ & 11 \\
\hline & 185 & $R(-1)$ & $\mathbf{R}$ & $\mathrm{R}(-1)$ & 16 & 33 & - & 00 & $X^{\prime}=-x$ & 2 \\
\hline & 386 & $R(-1)$ & $\mathbf{R}$ & $R(+1)$ & 15 & 12 & - & 00 & $x^{1}=-x /(b+2)$ & 36 \\
\hline
\end{tabular}




\begin{tabular}{|c|c|c|c|c|c|c|c|c|c|c|}
\hline ISEG & JSEG & $\operatorname{IS}(\Delta \mathrm{b})$ & by & $F S(A b)$ & next & $s^{\prime} s$ & tracks & kj cond & coefficients & code \\
\hline \multirow[t]{7}{*}{17} & 187 & $R(+1)$ & $\underline{\mathbf{R}}$ & $o(0)$ & 0 & 20 & - & 00 & $X^{\prime}=X$ & 1 \\
\hline & 188 & $R(+1)$ & $\underline{\mathrm{R}} / \mathrm{WR}$ & $0(0)$ & 0 & 31 & $(3,2)$ & 00 & $X^{1}=X A(1,0)$ & $\mathbf{5}$ \\
\hline & 189 & $R(+1)$ & $\mathbf{R}$ & $R(+1)$ & 17 & 00 & - & 00 & $X^{\prime}=x$ & $\mathbf{I}$ \\
\hline & 190 & $R(+1)$ & $\mathbf{R}$ & $R(+1)$ & 17 & 11 & - & 00 & $X^{\prime}=X C(0)$ & 6 \\
\hline & 191 & $R(+1)$ & $\mathbf{R}$ & $R(+1)$ & 17 & 22 & - & 00 & $X^{\prime}=-X$ & 2 \\
\hline & 192 & $R(+1)$ & $\mathbf{R}$ & $R(+1)$ & 17 & 33 & - & $\mathbf{0 0}$ & $x^{\prime}=-x$ & 2 \\
\hline & 193 & $R(+1)$ & $\mathbf{R}$ & $R(-1)$ & 18 & 21 & - & 00 & $x^{\prime}=x / b$ & 9 \\
\hline \multirow[t]{7}{*}{18} & 194 & $R(-1)$ & $\underline{\mathbf{R}}$ & $0(0)$ & 0 & 10 & - & 00 & $x^{1}=x$ & 1 \\
\hline & 195 & $R(-1)$ & $\underline{\mathrm{R} / W R}$ & $o(0)$ & 0 & 32 & $(3,2)$ & 00 & $X^{\prime}=X A(1,2)$ & 10 \\
\hline & 196 & $R(-1)$ & $\mathbf{R}$ & $R(-1)$ & 18 & 00 & - & 00 & $X^{*}=x$ & 1 \\
\hline & 197 & $R(-1)$ & $\mathbf{R}$ & $R(-1)$ & 18 & 11 & - & 00 & $X^{\prime}=-X$ & 2 \\
\hline & 198 & $R(-1)$ & $\mathbf{R}$ & $\mathrm{R}(-1)$ & 18 & 22 & - & 00 & $x^{\prime}=X C(2)$ & 11 \\
\hline & 199 & $\mathrm{R}(-1)$ & $\mathbf{R}$ & $R(-1)$ & 18 & 33 & - & 00 & $x^{\prime}=-x$ & 2 \\
\hline & 200 & $R(-1)$ & $\mathbf{R}$ & $R(+1)$ & 17 & 12 & - & 00 & $x^{1}=-x /(b+2)$ & 36 \\
\hline
\end{tabular}




\begin{tabular}{|c|c|c|c|c|c|c|c|c|c|c|}
\hline ISEG & JSEG & $\operatorname{IS}(\Delta \mathrm{b})$ & by & $\mathrm{FS}(\Delta \mathrm{b})$ & next & s's & tracks & kjcond & coefficients & code \\
\hline \multirow[t]{7}{*}{19} & 201 & $E(-1)$ & $\underline{\mathbf{L}}$ & $0(0)$ & 0 & 02 & $=$ & 00 & $X^{1}=x$ & 1 \\
\hline & 202 & $L(-1)$ & $\underline{L}$ & $B(0)$ & 0 & 13 & - & 00 & $x^{\prime}=x A(2,1)$ & 4 \\
\hline & 203 & $L(-1)$ & $\mathrm{L}$ & $L(-1)$ & 19 & 00 & - & 00 & $X^{\prime}=\mathrm{X}$ & 1 \\
\hline & 204 & $L(-1)$ & $\mathbf{L}$ & $L(-1)$ & 19 & 11 & - & 00 & $x^{\prime \prime}=x C(1)$ & 37 \\
\hline & 205 & $L(-1]$ & $\mathbf{L}$ & $L(-1)$ & 19 & 22 & $=$ & 00 & $x^{\prime}=-x$ & 2 \\
\hline & 206 & $L(-1)$ & $\mathbf{L}$ & $\mathbf{E}(-1)$ & 19 & 33 & * & 00 & $x^{\prime}=-x$ & 2 \\
\hline & 207 & $L(-1)$ & L & $L(+1)$ & 20 & 12 & - & 00 & $x^{\prime}=x /(b+1)$ & 38 \\
\hline \multirow[t]{7}{*}{20} & 208 & $L(+1)$ & $\underline{\mathbf{L}}$ & $0(0)$ & 0 & 01 & - & 00 & $x^{\prime}=x$ & 1 \\
\hline & 209 & $L(+1)$ & $\underline{L}$ & $0(0)$ & 0 & 23 & - & 00 & $X^{\prime}=X A(0,1\}$ & 3 \\
\hline & 210 & $L(+1)$ & $\mathbf{L}$ & $L(+1)$ & 20 & 00 & - & 00 & $X^{1}=X$ & 1 \\
\hline & 211 & $L(+1)$ & L & $L\{+1\}$ & 20 & 11 & - & 00 & $x^{\prime}=-x$ & 2 \\
\hline & 212 & $L(+1)$ & $L$ & $L(+1)$ & 20 & 22 & - & 00 & $x^{\prime}=x C(1)$ & 37 \\
\hline & 213 & $L(+1)$ & $\mathbf{l}$ & $L(+1)$ & 20 & 33 & $=$ & 00 & $x^{\prime}=-x$ & 2 \\
\hline & 214 & $L(+1)$ & L & $L(-1)$ & 19 & 21 & - & 00 & $x^{\prime}=-x /(b+1)$ & 39 \\
\hline
\end{tabular}




\begin{tabular}{|c|c|c|c|c|c|c|c|c|c|c|}
\hline ISEG & JSEG & $\operatorname{IS}(\Delta \mathrm{b})$ & by & $\mathrm{FS}(\Delta \mathrm{b})$ & next & $\mathrm{s}^{\prime} \mathrm{s}$ & tracks & $k j \operatorname{cond}$ & coefficients & code \\
\hline \multirow[t]{7}{*}{21} & 215 & $R(+1)$ & $\underline{\mathbf{R}}$ & $0(0)$ & 0 & 20 & - & 00 & $X^{\prime}=X$ & 1 \\
\hline & 216 & $\mathrm{R}(+1)$ & $\underline{\mathbf{R}}$ & $0(0)$ & 0 & 31 & - & 00 & $X^{\prime}=X A(1,0)$ & 5 \\
\hline & 217 & $R(+1)$ & $\mathbf{R}$ & $\mathrm{R}(+1)$ & 21 & 00 & - & 00 & $x^{\prime}=x$ & 1 \\
\hline & 218 & $R(+1)$ & $\mathbf{R}$ & $\mathbf{R}(+1)$ & 21 & 11 & $*$ & 00 & $X^{\prime}=X C(0)$ & 6 \\
\hline & 219 & $R(+1)$ & $\mathbf{R}$ & $R(+1)$ & 21 & 22 & - & 00 & $X^{\prime}=-X$ & 2 \\
\hline & 220 & $R(+1)$ & $\mathbf{R}$ & $R(+1)$ & 21 & 33 & - & 00 & $X^{t}=-x$ & 2 \\
\hline & 221 & $R(+1\}$ & $\mathbf{R}$ & $\mathrm{R}(-1)$ & 22 & 21 & - & 00 & $X^{\prime}=X / b$ & 9 \\
\hline \multirow[t]{7}{*}{22} & 222 & $R(-1)$ & $\underline{\mathbf{R}}$ & $0(0)$ & 0 & 10 & - & 00 & $X^{\prime}=X$ & 1 \\
\hline & 223 & $R(-1)$ & $\underline{\mathbf{R}}$ & $0(0)$ & 0 & 32 & - & 00 & $X^{i}=X A(1,2)$ & 10 \\
\hline & 224 & $R(-1)$ & $\mathbf{R}$ & $R(-I)$ & 22 & 00 & - & 00 & $x^{\prime}=x$ & $\mathbf{1}$ \\
\hline & 225 & $R(-1)$ & $\mathbf{R}$ & $R(-1)$ & 22 & 11 & - & 00 & $X^{\prime}=-x$ & 2 \\
\hline & 226 & $R(-1)$ & $\mathbf{R}$ & $R(-1)$ & 22 & 22 & - & $\infty$ & $X^{\prime}=x C(2)$ & 11 \\
\hline & 227 & $R(-1)$ & $\mathbf{R}$ & $\mathbf{R}(-1)$ & 22 & 33 & - & 00 & $X^{4}=-x$ & 2 \\
\hline & 228 & $R(-1)$ & $\mathbf{R}$ & $R(+1)$ & 21 & 12 & - & 00 & $x^{1}=-x /(b+2)$ & 36 \\
\hline
\end{tabular}


Table V. Loop Statistics for Given Examples

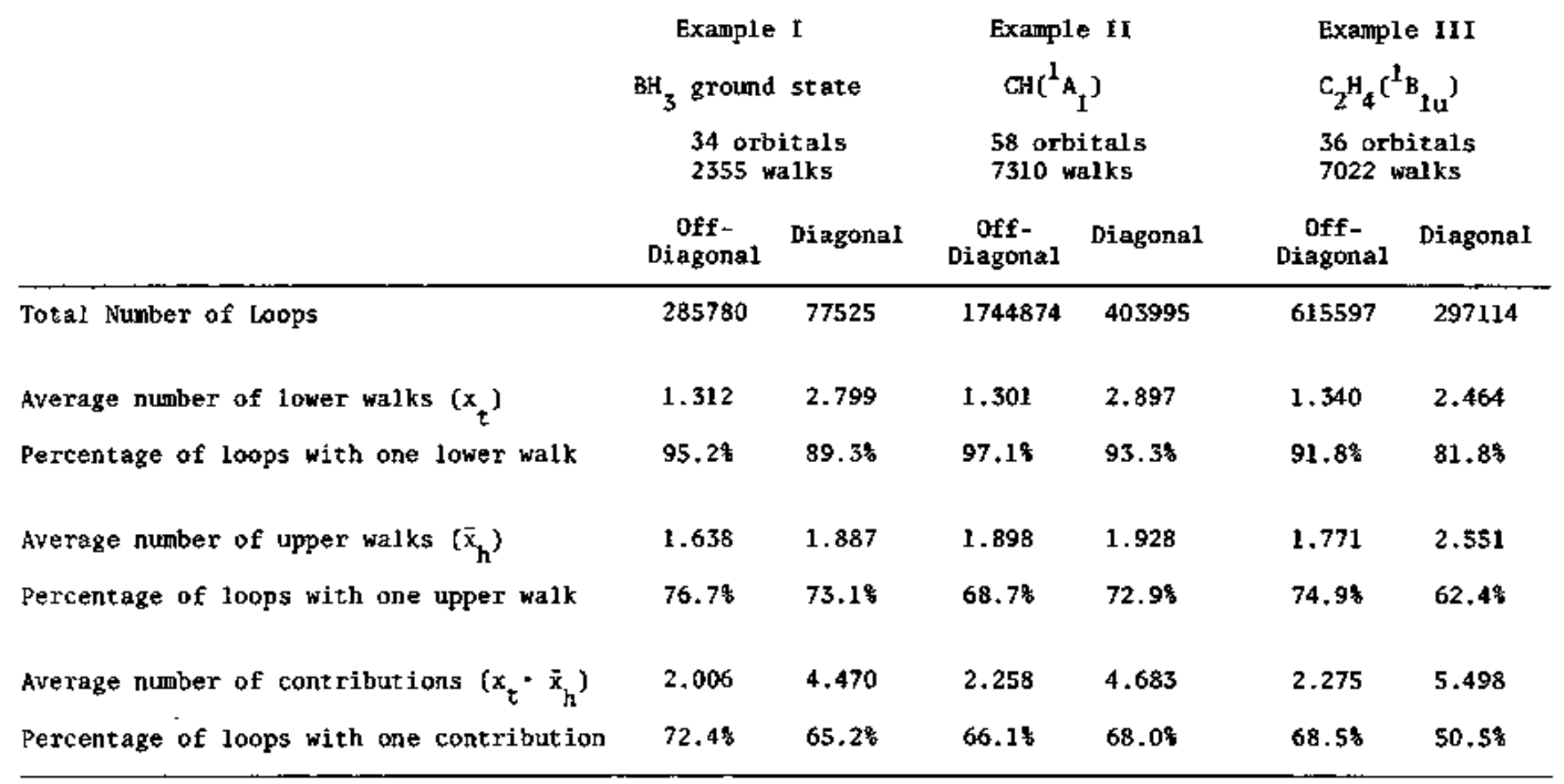


Table VI. Theoretical results (in eV) for the vertical electronic spectrun of ketene. The entries for DZ + Rydberg SCF and DZ + Polarization SCF are changes relative to the DZ SCF excitation energies.

\begin{tabular}{|c|c|c|c|c|c|c|}
\hline State & $\begin{array}{l}\text { Doubie Zeta }(\mathrm{DZ})^{2} \\
\text { Self-Consistent- } \\
\text { Field }\end{array}$ & $\begin{array}{l}\text { Double Zeta } \\
\text { Unitary CI }\end{array}$ & $\underset{\text { SCF }}{D z}+\underset{\text { Rydberg }}{ }$ & 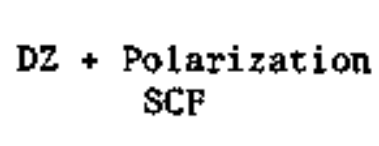 & Experiment ${ }^{b}$ & Harding-Goddard ${ }^{c}$ \\
\hline & 0 & 0 & 0 & 0 & 0 & 0 \\
\hline${ }^{3} A_{2}$ & 2.98 & 3.58 & -0.06 & +0.18 & - & 3.62 \\
\hline${ }^{{ }^{1}} A_{2}$ & 3.17 & 3.63 & -0.05 & +0.23 & 3.7 & 3.69 \\
\hline${ }^{3} A_{1}$ & 4.70 & 5.39 & -0.08 & +0.34 & 5.3 & 5.39 \\
\hline
\end{tabular}

${ }^{\text {a }}$ Reference 69.

$b_{\text {Reference } 72 .}$

Ceference 71 


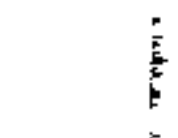

$$
1
$$$$
\text { , }
$$

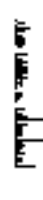


This report was done with support Irom the Department of Energy Any conclusions or opioiont expressed in this report represent solely thase of the aul horig) and nol neces5arly those of The Regents of the Universtty of Califernta, the Lawrence Berkeky Laboratory or the Departinent of Energy

Reference to a company or producl name toes not imply approval or recommendalion of the product by the Untversily of Calfornta or the U S Department of Energy Io the exclusion of others that may be suttabie

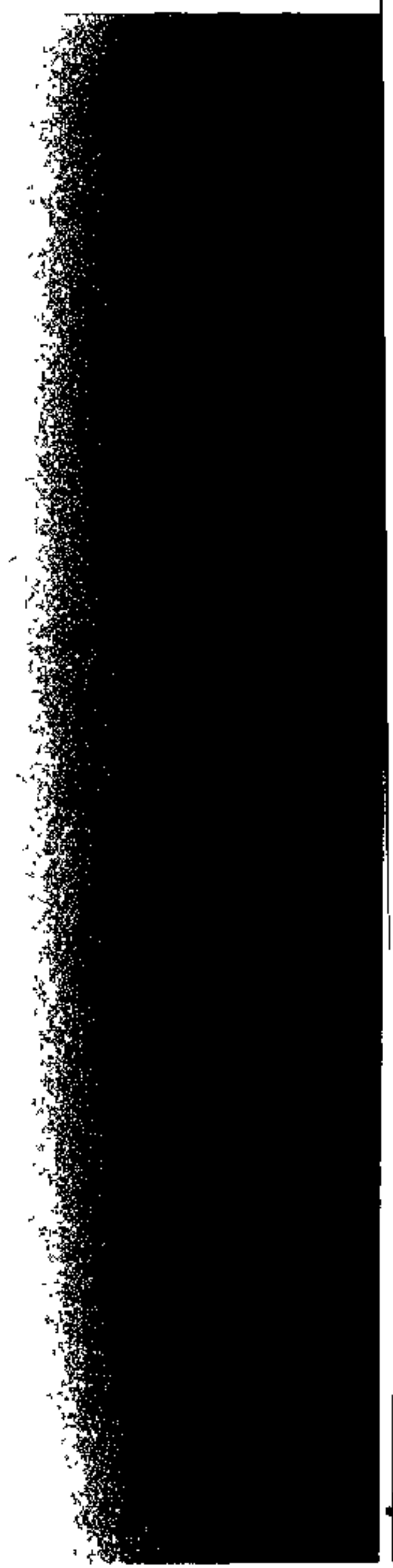

